\title{
1 Unsupervised Spatially Embedded Deep Representation of Spatial
}

\section{Transcriptomics}

4 Huazhu $\mathrm{Fu}^{1,{ }^{*}}$, Hang $\mathrm{Xu}^{2,{ }^{*}}$, Kelvin $\mathrm{Chong}^{2}$, Mengwei $\mathrm{Li}^{2}$, Kok Siong Ang ${ }^{2}$, Hong Kai Lee ${ }^{2}$,

5 Jingjing Ling ${ }^{2}$, Ao Chen ${ }^{3}$, Ling Shao ${ }^{1}$, Longqi Liu ${ }^{3}$, Jinmiao Chen ${ }^{2, \dagger}$

6

$7 \quad{ }^{1}$ Inception Institute of Artificial Intelligence, Abu Dhabi, United Arab Emirates

$8 \quad{ }^{2}$ Singapore Immunology Network (SIgN), Agency for Science, Technology and Research

9 (A*STAR), 8A Biomedical Grove, Immunos Building, 138648, Singapore

$10{ }^{3}$ BGI-ShenZhen, Shenzhen 518103, China.

$12{ }^{*}$ These authors contributed equally to this work.

13 †Corresponding author. Email: chen_jinmiao@immunol.a-star.edu.sg

16 Key words: spatial transcriptomics; graph convolutional network; gene expression; deep

17 learning 


\section{Abstract}

21 Spatial transcriptomics enable us to dissect tissue heterogeneity and map out inter-cellular 22 communications. Optimal integration of transcriptomics data and associated spatial 23 information is essential towards fully exploiting the data. We present SEDR, an unsupervised 24 spatially embedded deep representation of both transcript and spatial information. The 25 SEDR pipeline uses a deep autoencoder to construct a low-dimensional latent 26 representation of gene expression, which is then simultaneously embedded with the 27 corresponding spatial information through a variational graph autoencoder. We applied

28 SEDR on human dorsolateral prefrontal cortex data and achieved better clustering accuracy, 29 and correctly retraced the prenatal cortex development order with trajectory analysis. We 30 also found the SEDR representation to be eminently suited for batch integration. Applying

31 SEDR to human breast cancer data, we discerned heterogeneous sub-regions within a 32 visually homogenous tumor region, identifying a tumor core with pro-inflammatory 33 microenvironment and an outer ring region enriched with tumor associated macrophages

34 which drives an immune suppressive microenvironment. 
40 Single-cell omics technologies enable measurements at single-cell resolution, and have led

41 to discoveries of new subpopulations across various tissues, in both healthy and diseased

42 states. However, the dissociation of tissue into single cells prior to high throughput omics

43 data acquisition leads to cellular spatial information being lost, hindering our ability to dissect

44 the spatial organization and intercellular interactions of individual cells. While computational

45 tools have been developed to predict cell-cell interactions from ligand and receptor

46 expression, they require validation using immunohistochemistry (IHC) or

47 immunofluorescence (IF). Emerging spatial omics technologies overcome these limitations

48 through the simultaneous measurement of gene/protein expression and spatial locations of

49 cells. Such spatially resolved transcriptomes of histological tissues enable the reconstruction

50 of tissue architecture and cell-cell interactions. ${ }^{1,2,3,4,5,6,7,8,9}$ This approach has proven

51 valuable in many applications including studies on brain disorders, ${ }^{2,10}$ tumour

52 microenvironments, ${ }^{3,11}$ and embryonic development. ${ }^{12}$

53 Among currently available spatial transcriptomics approaches, in situ capturing-based

54 technologies such as 10x Genomics Visium and Nanostring GeoMX DSP have gained

55 popularity owing to their accessibility and ability to profile a large number of mRNA targets

56 within each spot. In principle, a histological section from a tissue sample is permeabilized

57 and the released mRNA is captured by either spatially arrayed oligos on slide surfaces or by

58 pre-hybridized RNA-target barcodes in manually defined regions of interest (ROIs). However,

59 both technologies suffer from mRNA capture area limitations, with the smallest diameter

60 typically being $\sim 50 \mu \mathrm{m}$, which is larger than a single cell. To overcome this, several

61 computational methods have been developed to deconvolve the cell mixture of the spatial

62 spot. ${ }^{13,14,15,16,17,18,19,20}$ Recently, improvements in mRNA capture methods have led to smaller

63 subcellular capture areas that are $\sim 1-10 \mu \mathrm{m}$ in diameter. These high-resolution spatial

64 transcriptomics methods can obtain spatially resolved transcriptomes with increased spatial

65 fidelity, without compromising the number of genes captured. They include Slide-seq, ${ }^{8}$ DBiT- 
66 seq, ${ }^{9}$ Stereo-seq, ${ }^{5}$ PIXEL-seq,${ }^{6}$ and Seq-Scope, ${ }^{7}$ with the highest resolution $(\sim 1 \mu \mathrm{m})$ thus far

67 obtained by the latter three. These submicrometer-resolution methods usually require voxel

68 binning or cell segmentation to produce a gene-by-cell expression matrix for downstream

69 analysis. Capture area sizes have also improved and thus increased the overall cell

70 throughput, necessitating new computational methods that can handle big spatial data.

71 When analyzing spatial transcriptomics data, combining both gene expression and

72 spatial information to learn a discriminative representation for each cell or spot is crucial.

73 However, established workflows, e.g., Seurat, ${ }^{21}$ still employ pipelines designed for single-cell

74 RNA-seq analysis, which primarily focus on gene expression data and ignore the structural

75 relationship of the spatial neighborhood. Recently, several new methods have been

76 developed for spatial transcriptomics to overcome this limitation. For example,

77 BayesSpace ${ }^{22}$ starts from a Markov random field (MRF) prior which hypothesizes that spots

78 belonging to the same cell type should be closer to one another, and updates the model with

79 a Bayesian approach. Giotto ${ }^{23}$ implements a hidden Markov random field (HMRF) model to

80 detect domains with coherent patterns by comparing gene expression between cells and

81 their neighbors. SpaGCN ${ }^{24}$ combines spatial distances and histological dissimilarities to

82 construct a weighted graph of spots, and then integrates the graph with gene expression

83 using a graph convolutional network (GCN) to cluster the spots. stLearn ${ }^{25}$ utilizes a deep

84 learning model on the spot images to extract morphological features, on which

85 morphological distances are calculated. It then uses the morphological distance and spatial

86 neighborhood information to normalize the gene expression of each spot based on its

87 identified neighbors. The normalized gene expression is then used as input for linear

88 principal component analysis (PCA), followed by uniform manifold approximation and

89 projection (UMAP), and spatial clustering. Notably, these methods mainly rely on PCA to

90 extract the highly variable features of gene expression data, which involves a linear

91 transformation, so they are unable to model complex non-linear relationships. While stLearn

92 does utilize deep learning, it is only applied to the image modality, and the model still relies

93 on linear PCA to extract features from the spatially normalized gene expression data. 
94 Moreover, these methods do not produce low-dimensional representations of jointly

95 embedded gene expression and spatial information. The joint embedding of gene expression

96 and spatial information is essential to effectively integrate both modalities for better

97 visualization, clustering, trajectory inference, and batch integration.

98 In this work, we developed an unsupervised spatially embedded deep representation

99 (SEDR) method for learning a low-dimensional latent representation of gene expression

100 embedded with spatial information. Our SEDR model consists of two main components, a

101 deep autoencoder network for learning a gene representation, and a variational graph

102 autoencoder network for embedding the spatial information. These two components are

103 optimized jointly to generate a latent representation for spatial transcriptomics data analysis.

104 We applied SEDR on the 10x Genomics Visium spatial transcriptomics and Stereo-seq

105 datasets and demonstrated its ability to achieve better representations for various follow-up

106 analysis tasks, including clustering, visualization, trajectory inference, and batch effects

107 correction.

108 Results

109 Overview of SEDR.

110 SEDR learns a gene representation in a low-dimensional latent space with jointly embedded

111 spatial information (Figure 1). Given spatial transcriptomics data, SEDR first learns a

112 nonlinear mapping from the gene expression space to a low-dimensional feature space using

113 a deep autoencoder network. Simultaneously, a variational graph autoencoder is utilized to

114 aggregate the gene representation with the corresponding spatial neighborhood relationships

115 to produce a spatial embedding. Then, the gene representation and spatial embedding are

116 concatenated to form the final latent representation used to reconstruct the gene expression.

117 Thereafter, an unsupervised deep clustering method ${ }^{26}$ is employed to enhance the

118 compactness of the learned latent representation. This iterative deep clustering generates a

119 form of soft clustering that assigns cluster-specific probabilities to each cell, leveraging on 
120 the inferences between cluster-specific and cell-specific representation learning. Finally, the

121 learned latent representation can be applied towards various analysis tasks.

122 Quantitative assessment of SEDR on human dorsolateral prefrontal cortex (DLPFC)

123 dataset.

124 To perform a quantitative comparison of SEDR with other methods, we downloaded the 10x

125 Genomics Visium spatial transcriptomics data and the manually annotated layers for LIBD

126 human dorsolateral prefrontal cortex (DLPFC) data. ${ }^{2}$ The LIBD data includes 12 slices from

127 the human DLPFC, which span six cortical layers plus white matter. We chose this dataset

128 because the human DLPFC has clear and established morphological boundaries which can

129 serve as the ground truth. We first applied the standard Seurat pipeline ${ }^{21}$ to process and

130 cluster cells using only expression profiles and set the result as the benchmarking baseline

131 to investigate the extent to which spatial information improves cell clustering. As Giotto, ${ }^{23}$

132 stLearn, ${ }^{25}$ SpaGCN, ${ }^{24}$ and BayesSpace ${ }^{22}$ integrate spatial information and RNA-seq data for

133 clustering, we also applied them with recommended default parameters to the same dataset

134 for benchmarking against SEDR.

In brain slice 151673 (Figure 2A) with 3,639 spots and 33,538 genes, SEDR and

136 BayesSpace achieved the best performance in terms of both layer borders and adjusted rand

137 index (ARI). When comparing the results on all 12 DLPFC samples, SEDR had the second

138 highest mean ARI (0.427) (Figure 2A, bottom right), but the difference between SEDR and

139 the top performer BayesSpace (0.457) was not significant (Mann-Whitney $U$ Test: ${ }^{27} \mathrm{p}$ -

140 value=0.55). It should be noted that BayesSpace's clustering algorithm is optimized for

141 spatial omics, while SEDR is a dimension reduction method with its objective being to find

142 the best latent representation. SEDR followed by Leiden clustering, which was not

143 specifically designed or optimized for clustering spatial omics, achieved comparable

144 clustering performance to BayesSpace. This indicated that SEDR latent representations

145 effectively integrate gene expressions and spatial information for capturing inter-cluster

146 differences. Coupling SEDR with clustering algorithms that are better-suited for spatial omics 
147 can be expected to further improve the clustering accuracy. Furthermore, in contrast to

148 BayesSpace, which does not produce latent representations, SEDR-derived embeddings can

149 be used for not only clustering but also various downstream analysis tasks such as UMAP

150 visualization, trajectory inference, and batch effect correction, thus providing more flexibility

151 and utility. Similar to SEDR, SpaGCN also uses a GCN to process spatial transcriptomics

152 data. Moreover, it incorporates histological information, which is not included in SEDR.

153 However, the clustering performance of SEDR is better than that of SpaGCN (Mann-Whitney

$154 \cup$ Test, $p$-value < 0.05). stLearn also integrates histological data, but the performance is

155 likewise poorer. This may indicate that the current approaches utilized by SpaGCN and

156 stLearn to incorporate histological data are not optimal. To make full use of the histological

157 information, we may need to treat it as a separate data modality and use dedicated multi-

158 view algorithms for integration.

159 SEDR generates a set of low-dimensional representation features which can be used

160 in various downstream analyses, such as trajectory inference. ${ }^{28}$ In our experiments, we used

161 Monocle $^{29}$ to perform trajectory inference on sample 151673 with the Seurat output (RNA-

162 only) and the low-dimensional SEDR representation features. We found that SEDR showed

163 significantly improved performance over Seurat (Figure 2B). In the UMAP plot of SEDR's

164 output, cells belonging to different layers were well-organized, and when we selected white

165 matter (WM) as the root, the pseudo-time reflected the correct "inside-out" developmental

166 ordering of cortical layers (Figure 2B). This demonstrated that, compared to RNA-only

167 analyses, incorporating spatial information enabled SEDR to generate a better latent

168 representation summarizing the spatial transcriptomics data. We further confirmed our

169 observations with another trajectory inference method named partition-based graph

170 abstraction (PAGA), ${ }^{30}$ using the SEDR-derived latent space embedding instead of UMAP

171 coordinates (Figure $2 \mathrm{C}$ ). The PAGA results showed that adjacent cortical layers tend to

172 share greater similarity, suggesting that spatial adjacency is linked with transcriptomic and

173 even functional similarity. Notably, the trajectory was concordant with the chronological order

174 of cortex development..$^{31,32,33}$ We then compared the PAGA graphs generated using the 
175 Seurat-derived principal components and SEDR embeddings. For each of the 12 DLPFC

176 slices, we calculated the ratio of the edge weights between adjacent cortical layers to the

177 total sum of the weights of all edges. We found a significantly higher ratio for SEDR

178 compared to Seurat (Mann-Whitney U test p-value < 0.05) (Figure 2C, bottom).

\section{SEDR corrects for batch effects.}

180 The proliferation of spatial omics applications is generating ever increasing volumes of

181 spatially resolved omics data across different labs. However, differences in protocols and

182 technologies complicate comparisons and data integration when trying to achieve consensus

183 on spatially resolved tissue atlases. As with single-cell RNA-seq (scRNA-Seq), removing

184 batch effects in spatial omics datasets is a significant challenge. To date, no methods are

185 available for this. Here, we demonstrate that SEDR can learn joint embeddings across

186 multiple batches and project them into a shared latent space. Furthermore, SEDR employs a

187 deep embedded clustering (DEC) loss function that enables it to retain biological variations

188 while reducing technical variations. We evaluated the batch correcting performance of SEDR

189 on the DLPFC datasets. We first assessed the batch variations among the twelve datasets

190 and selected three sets $(151507,151672,151673)$ which exhibited substantial batch effects.

191 The common cortical layers from different batches were separated, as shown in the UMAP

192 plot (Figure 3A). We first applied Harmony to remove the batch effects due to its superior

193 performance in scRNA-seq data integration. ${ }^{34}$ Harmony was able to mix batches while

194 keeping different layers apart. However, when we zoomed into the individual layers, we

195 found distinct batch-specific subclusters, suggesting that the batch effects were not

196 completely removed (Figure 3B). Next, we tested SEDR and found that the batch effects

197 were substantially reduced (Figure 3C). Common layers across batches were brought very

198 close and were well-aligned, while different layers were minimally mixed. Further application

199 of Harmony on the SEDR embeddings evenly mixed the batches while maintaining

200 separation between layers (Figure 3D). Notably, batch-specific clusters were no longer

201 present within individual layers. This showed that the combination of SEDR with Harmony 
202 effectively removed the batch effects. Among the other spatial omics analysis methods, only

203 stLearn is able to produce a latent space embedding which can be fed to Harmony for batch

204 correction. Therefore, we benchmarked SEDR against stLearn. As stLearn is unable to jointly

205 project different batches to a shared latent space due to its requirement of histological

206 images as input, we generated latent space embeddings from each dataset and then

207 concatenated them for Harmony integration. The results showed that batches were not well

208 mixed and the layers were poorly separated (Figure 3E). In conclusion, SEDR combined with

209 Harmony outperforms both Harmony alone and stLearn with Harmony, and can serve as an

210 effective method for batch correction of spatial omics data.

211 Dissecting tumor heterogeneity and immune microenvironments using SEDR.

212 Intratumoral heterogeneity in cancer complicates effective treatment formulations and is

213 associated with poor survival prospects. ${ }^{35}$ Spatial transcriptomics is an effective tool for

214 dissecting and characterizing intratumoral heterogeneity and tumor-immune crosstalk. We

215 tested SEDR on the 10x Visium spatial transcriptomics data for human breast cancer, which

216 is known for its high intratumoral and intertumoral differences. To aid in the interpretation of

217 SEDR results, we performed manual pathology labeling based on H\&E staining. It should be

218 noted that, unlike the cerebral cortex which has clear and established morphological

219 boundaries, tumor tissues are highly heterogeneous and encompass complex

220 microenvironments. Manual labeling solely based on tumor morphology is inadequate for

221 characterizing such complexity. Based on pathological features, we manually segmented the

222 histological image into twenty regions, which we then grouped into four main morphotypes:

223 ductal carcinoma in situ/lobular carcinoma in situ (DCIS/LCIS), healthy tissue (Healthy),

224 invasive ductal carcinoma (IDC), and tumor surrounding regions with low features of

225 malignancy (Tumor edge) (Figure 4A top left, Supplementary Figure 1A). Visually, all five

226 methods agreed with the manual annotations at the macroscopic level (Figure 4A).

227 Nevertheless, the SEDR clusters presented a smoother segmentation compared to other

228 methods, while those derived by Seurat, stLearn, and SpaGCN appeared fragmented with 
229 irregular boundaries. Notably, SEDR found more sub-clusters within the tumor regions, while

230 other methods were prone to dividing the healthy regions into subclusters, given that all

231 methods were set to generate the same number of clusters. Specifically, within the

232 seemingly homogenous tumor region DCIS/LCIS_3, SEDR separated an outer "ring" (Figure

2334 A, SEDR cluster 7) from the tumor core (Figure 4A, SEDR cluster 3). These SEDR clusters

234 indicated transcriptionally and spatially distinct compartments within the visually homogenous

235 tumor regions. In addition to clustering analysis, we also employed the Seurat3 'anchor'-

236 based integration workflow to perform probabilistic transfer of annotations from scRNA-seq

237 reference data for human breast $\operatorname{cancer}^{36}$ to the spatial data. For each spot, we obtained a

238 probabilistic classification for each of the scRNA-seq derived classes (Figure 4B,

239 Supplementary Figure 1B). The transferred class probabilities were able to delineate the

240 tumor regions and regions where immune cells or fibroblasts were present, which were

241 useful for further dissecting the tumor microenvironment.

242 To further characterize the transcriptional differences between SEDR cluster 3 (tumor

243 core) and cluster 7 (tumor edge) of DCIS/LCIS_3 region, we performed differential

244 expression analysis followed by pathway enrichment analysis (Figure 4C). In cluster 3, we

245 observed the upregulation of interferon signaling pathways (IFIT1, IFITM1, IFITM3 and

246 TAP1) and NK or neutrophil activities (FCGR3B and TNFSF10) (Figure 4C, Supplementary

247 Figure 2E). In addition, $\mathrm{RHOB}$ was upregulated in this region, pointing towards reduced

248 metastatic potential. ${ }^{40}$ Cluster 3 represented a region where cancer growth was limited by

249 pro-inflammatory immune responses. On the other hand, in cluster 7 , we observed the

250 presence of TAMs (Figure 4B, Supplementary Figure 2D), memory B cells (IGHG1, IGHG3,

251 IGHG4, IGLC2 and IGLC3) and fibroblasts (COL1A1, COL1A2, COL3A1, COL5A1,

252 COL6A1, COL6A2 and FN1) (Figure 4C, Supplementary Figure 2E). Upregulated cathepsin

253 activity (CTSB, CTSD and CTSZ) and complement pathway (C1QA, C1S) indicated pro-

254 tumor activity by the TAMs in this region. ${ }^{41,42,43}$ Upregulation of actin cytoskeleton signalling

255 also suggested higher metastasis potential of cluster 7 (Figure 4C). Moreover, upregulated

256 cathepsin activity and metalloproteinase inhibitors (TIMP1 and TIMP3) also indicated 
257 disturbance in the extracellular matrix integrity (Supplementary Figure 2E). Overall, cluster 7

258 represented a region with an immune-suppressed pro-tumor microenvironment and high 259 potential for cancer metastasis.

260 A number of driving forces have been hypothesized as responsible for the metastatic 261 transition of tumor cells from a pre-invasive state to invasive carcinoma, including pro-tumor 262 immune microenvironments and reduced cell-cell interactions within the tumor. ${ }^{37}$ In this 263 study, we employed PAGA to infer the inter-relatedness between the manually annotated 264 tumor regions to trace the metastatic transition process. The PAGA graph generated using 265 the SEDR embeddings suggested that DCIS_LCIS_3 was related to the neighboring IDC_6 266 region (Figure 4D). The differentially expressed genes (DEGs) and enriched pathways of 267 DCIS_LCIS_3 compared to all other DCIS_LCIS regions showed that DCIS_LCIS_3 had 268 more immune infiltrates (Supplementary Figure 2A, 2B, 2C), particularly tumor associated 269 macrophages (TAMs) (Figure 4B, top), while the other DCIS_LCIS regions were mainly 270 comprised of actively dividing/cycling epithelial cells (Figure 4B, bottom) with upregulated 271 glycolytic and metabolic processes (Supplementary Figure 2C). TAM infiltration is known to 272 be strongly associated with poor survival rate in solid tumor patients due to its promotion of 273 tumor angiogenesis and induction of tumor migration, invasion and metastasis. ${ }^{38,39}$ We thus 274 performed Monocle3 analysis to infer the pseudo-time of the transition from DCIS_LCIS_3 to 275 IDC_6. As DCIS_LCIS_3 and IDC_6 coincided with SEDR clusters 3, 7, and 11 (Figure 4A, 276 4D), we applied Monocle3 on these three clusters and set cluster 3 (tumor core) as the starting point (Figure 4D bottom). Monocle3 analysis showed that pseudo-time derived from

278 SEDR embeddings better traced the inside-out tumor progression compared to that from 279 Seurat PCA embeddings. We subsequently identified genes that changed expression along 280 the Monocle3 pseudo-time and revealed sequential waves of gene regulation along the 281 trajectory (Figure 4E).

282 In summary, SEDR analysis revealed intratumoral heterogeneity within visually 283 homogenous tumor regions and revealed the tumor outer ring (cluster 7) with TAM infiltration 284 and cancer associated fibroblasts (CAFs), both of which have been reported to facilitate 
285 tumor spread. ${ }^{44,45}$ SEDR also enabled the mapping of a molecular trajectory from the tumor

286 core to its neighboring invasive region, demonstrating the transition from a pro-inflammatory

287 to an immune-suppressive microenvironment, which may contribute to tumor metastasis.

288 SEDR can handle spatial transcriptomics of high resolution.

289 Currently available spatial omics technologies, including 10x Visium Spatial Omics,

290 Nanostring GeoMX DSP, SLIDE-seq ${ }^{4}$, and DBIT-seq ${ }^{46}$, do not provide single-cell resolution,

291 with each capture spot containing 1 to 10 cells. However, newly emerging methods such as

292 Stereo-seq $^{5}$, PIXEL-Seq ${ }^{6}$, and Seq-Scope ${ }^{7}$ can achieve submicrometer and thus subcellular

293 resolution. With continued technology advancement, the spatial resolution and number of

294 cells detected per tissue will significantly improve, producing large datasets with high

295 throughput. As such, we evaluated SEDR's performance on one type of such data produced

296 by Stereo-seq from mouse olfactory bulb tissues (Figure 5). The coronal section of a mouse

297 olfactory bulb contains the olfactory nerve layer (ONL), glomerular layer (GL), external

298 plexiform layer (EPL), mitral cell layer (MCL), internal plexiform layer (IPL), granule cell layer

299 (GCL), and rostral migratory stream (RMS) (Figure 5A). We performed unsupervised

300 clustering using the Seurat-derived principal components and SEDR-derived embeddings to

301 computationally reconstruct the spatial identity of the olfactory bulb tissues. Compared to

302 Seurat clusters, those produced by SEDR better reflected tissue organization and were more

303 consistent with known anatomical layers (Figure 5B, 5C). We also performed quantitative

304 assessment using local inverse Simpson's index (LISI) and found that SEDR produced

305 significantly lower LISI than Seurat, implying better spatial separation by SEDR (Figure 5D).

306 Discussion

308 Cell type heterogeneity is a feature of both healthy and diseased tissue. Capturing this 309 heterogeneity, coupled with its spatial arrangement in the tissue, is crucial when studying the 310 roles of cells and their cross-talk. Spatial omics technologies represent the state-of-the-art 311 approaches for capturing omics data with corresponding spatial information from tissue 
312 samples. In this paper, we have introduced SEDR, which leverages on cutting edge graph

313 neural network techniques to achieve a better representation of spatial omics data that can

314 be used for clustering and further downstream analyses. SEDR first learns a low-dimensional

315 latent space representation of the transcriptome information with a deep autoencoder

316 network, which is then aggregated with spatial neighborhood information by a variational

317 graph autoencoder to create a spatial embedding. This spatial embedding is then

318 concatenated with the gene expression to be decoded to reconstruct the final gene

319 expression for further analyses. We first demonstrated the efficacy of SEDR in delineating

320 the different cerebral cortex layers with higher clarity than competing methods, and

321 recapitulated the associated development order by using the joint latent representation with

322 Monocle3.

To enhance the analytical power and resolution of spatial omics, we need to integrate

324 multiple datasets from the same tissue. Similar to single-cell transcriptomic data, spatial

325 omics datasets generated in different batches also contain batch-specific systematic

326 variations that present a challenge to batch-effect removal and data integration. In our study,

327 we demonstrated that by combining SEDR and Harmony, we were able to effectively remove

328 batch effects present. In the future, we will integrate Harmony into the SEDR workflow.

329 Spatial omics technologies such as Stero-seq are able to measure a large number of

330 cells in a single experiment through high spatial resolutions and large tissue sizes. In the

331 near future, we expect to see ever-increasing throughput from spatial omics experiments,

332 which will result in spatial omics big data that will pose significant challenges to data analysis

333 and integration. Computational methods that employ GCNs require the entire graph to be

334 loaded into GPU memory, which inhibits their application to very large datasets. We will

335 improve the memory efficiency of SEDR using a GCN mini-batch or parallel techniques to

336 construct large-scale graphs for spatial omics data of high throughput and resolution.

337 Furthermore, technologies with a capture spot size smaller than the diameter of a cell will

338 also require new computational methods that can accurately delineate cells based on 
339 capture spots. In the future, we will integrate cell segmentation based on H\&E or DAPI

340 staining into the SEDR workflow.

341 The current SEDR methodology focuses on gene expression and spatial information, 342 and does not make use of histological images. Contemporary methods such as SpaGCN and

343 stLearn use histological images as input, but in a suboptimal fashion, as demonstrated in our

344 study. Specifically, SpaGCN utilizes histological image pixels as features by calculating the

345 mean color values from the RGB channels directly. However, the pixel values are easily

346 affected by noise and cannot provide semantic features for cell analysis. A more effective

347 approach can be to adopt a deep CNN model which can learn high-level representations for

348 histological images. stLearn introduces a deep learning model to extract image features of

349 the spots and integrates them with the spatial location and gene expression. However,

350 stLearn employs a model pre-trained on natural images, and does not fine-tune the network

351 for histological images. In the future, we will incorporate histological images as an additional

352 modality into the SEDR model. We will add an image autoencoder network to learn image

353 features, and jointly learn the latent representation by integrating gene expression, image

354 morphology, and spatial information.

355 In summary, SEDR is a promising new approach that builds an integrated 356 representation of cells using both transcriptomic data and spatial coordinates. SEDR-derived 357 low-dimensional embedding enables more accurate clustering, trajectory inference and batch 358 effect correction. Our model is also able to handle spatial transcriptomics with capture spot 359 sizes ranging from $50 \mu \mathrm{m}$ to less than $1 \mu \mathrm{m}$. Furthermore, we applied SEDR on human breast 360 cancer to reveal heterogeneous sub-regions within the seemly homogenous tumor region 361 and shed light on the role of immune microenvironments on tumor invasiveness. 


\section{Dataset preprocessing.}

364 Our SEDR method takes spatial transcriptomic gene expressions and spatial coordinates as 365 inputs. The raw gene expression counts are first normalized using the respective library sizes 366 (by normalize_total in Scanpy (v.1.5.0)), with very highly expressed genes excluded when 367 computing the normalization factor (size factor) for each cell ${ }^{47}$. PCA is then applied to extract 368 the first 200 principal components to generate the initial gene expression matrix.

\section{Graph construction for spatial transcriptomics data.}

371 To create a graph representing the cell-cell spatial relationships in spatial transcriptomics 372 data, we calculate the Euclidean distances between cells using the image coordinates, and 373 select the top 10 nearest neighbors of each cell to construct an adjacency matrix. The 374 adjacency matrix, denoted by $A$, is a symmetric matrix, where $A_{i j}=A_{j i}=1$ if $i$ and $j$ are 375 neighbors, and 0 otherwise.

Deep autoencoder for latent representation learning.

377 The latent representation of gene expression is learned using a deep autoencoder. The 378 encoder part, consisting of two fully connected stacked layers, generates a low-dimensional 379 representation $Z_{f} \in \mathbb{R}^{N \times D_{f}}$ from the input gene expression matrix $X \in \mathbb{R}^{N \times M}$. Meanwhile, 380 the decoder part with one fully connected layer reconstructs the expression matrix $X^{\prime} \in$ $381 \mathbb{R}^{N \times M}$ from the latent representation $Z \in \mathbb{R}^{N \times D}$, which is obtained by concatenating the low382 dimensional representation $Z_{f}$ and spatial embedding $Z_{g} \in \mathbb{R}^{M \times D_{g}}$, where $N$ is the number of 383 cells, $M$ is the number of input genes, and $D_{f}, D_{g}, D$ are the dimensions of the low384 dimensional expression representation learned by the encoder, the spatial embedding 385 learned by the GCN, and the final latent representation of SEDR, respectively with $D=D_{f}+$ $386 D_{g}$. The objective function of the deep autoencoder maximizes the similarity between the 
387 input gene and reconstructed expressions measured by the mean squared error (MSE) loss

388 function $\sum\left(X-X^{\prime}\right)^{2}$.

389 Variational graph autoencoder for spatial embedding.

390 SEDR utilizes a variational graph autoencoder ${ }^{48}$ (VGAE) to embed the spatial information of neighboring cells. With the adjacency matrix $A$ and its degree matrix $D$, the VGAE learns a graph embedding $Z_{g}$ with the following format: $g:\left(A, Z_{f}\right) \rightarrow Z_{g}$, where $Z_{f}$ is the node/gene representation from the deep autoencoder. The inference part of the VGAE is parameterized by a two-layer $\mathrm{GCN}^{49}$ :

where $\mu=G C N_{\mu}\left(A, Z_{f}\right)$ is the matrix of mean vectors, and $\log \sigma=G C N_{\sigma}\left(A, Z_{f}\right)$. The twolayer GCN is defined as:

$$
\operatorname{GCN}\left(A, Z_{f}\right)=\tilde{A} \operatorname{ReLU}\left(\tilde{A} Z_{f} W_{0}\right) W_{1}
$$

399 with a weight $W_{i}$ and symmetrically normalized adjacency matrix $\tilde{A}=D^{-\frac{1}{2}} A D^{-\frac{1}{2}}$. The spatial embedding $Z_{g}$ and reconstructed adjacency matrix $A^{\prime}$ are generated as:

$$
A^{\prime}=\sigma\left(Z_{g} \cdot Z_{g}^{T}\right)
$$

with $Z_{g}=G C N\left(A, Z_{g}\right)$. The objective of the VGAE is to minimize the cross-entropy (CE) loss between the input adjacency matrix $A$ and reconstructed adjacency matrix $A^{\prime}$, while simultaneously minimizing the Kullback-Leibler $(\mathrm{KL})$ divergence between $g\left(Z_{g} \mid A, Z_{f}\right)$ and the Gaussian prior:

$$
p\left(Z_{g}\right)=\prod_{i} \mathcal{N}\left(z_{i} \mid 0, I\right)
$$

\section{Batch effect correction for spatial transcriptomics.}

408 Spatial relationships only exist within single spatial transcriptomic measurement; cells/spots 409 from different transcriptomic measurements have no direct spatial relation. Let $A^{k}$ and $Z_{f}^{k}$ 410 denote the adjacency matrix and deep gene representation of spatial omics $k$, we then 
411 create a block-diagonal adjacency matrix $A^{k}$ and concatenate the deep gene representation

412 in the cell dimension, as:

$$
A=\left[\begin{array}{ccc}
A^{1} & \cdots & 0 \\
\vdots & \ddots & \vdots \\
0 & \cdots & A^{K}
\end{array}\right], Z_{f}=\left[\begin{array}{c}
Z_{f}^{1} \\
\vdots \\
Z_{f}^{K}
\end{array}\right]
$$

414 where $K$ is the number of spatial omics. Based on this formulation, we transform different 415 spatial omics datasets (of potentially different sizes) into multiple graph instances in the form 416 of one block-diagonal adjacency matrices as inputs to SEDR.

417 To remove batch effects and enhance the compactness of its latent representation, SEDR 418 employs an unsupervised deep embedded clustering (DEC) method ${ }^{26}$ to iteratively group the 419 cells into different clusters. To initialize the cluster centers, we employ the KMeans of scikit420 learn on the learned latent representations. The number of clusters is pre-defined as a 421 hyperparameter. With the initialization, DEC improves the clustering using an unsupervised 422 iterative method of two steps. In the first step, a soft assignment $q_{i j}$ of latent point $z_{i}$ to 423 cluster center $\mu_{j}$ is calculated using the Student's t-distribution:

$$
q_{i j}=\frac{\left(1+|| z_{i}-\mu_{j}||^{2}\right)^{-1}}{\sum_{j \prime}\left(1+|| z_{i}-\mu_{j \prime} \|^{\prime}\right)^{2}} .
$$

In the second step, we iteratively refine the clusters by learning from their high confidence assignments with the help of an auxiliary target distribution $p$ based on $q_{i j}$ :

428 Based on the soft assignment $q_{i j}$ and auxiliary target distribution $p_{i j}$, an objective function is defined using the $\mathrm{KL}$ divergence:

$$
p_{i j}=\frac{q_{i j}^{2} / \sum_{i} q_{i j}}{\sum_{j \prime}\left(q_{i j}^{2} / \sum_{i} q_{i j \prime}\right)}
$$

431 The SEDR parameters and cluster centers are then simultaneously optimized by using 432 stochastic gradient descent (SGD) with momentum. 


\section{Seurat.}

435 Raw mRNA counts were preprocessed to remove low-quality genes and sctransformed to 436 remove technical artifacts and normalize the data. ${ }^{50}$ We then ran PCA to extract the top 30 437 principal components (PCs) and used them to calculate the shared nearest neighbors 438 (SNNs). Then, the Louvain clustering algorithm was used to identify clusters with the SNN 439 networks. We tried clustering at different resolutions to obtain the same number of clusters 440 as the ground truth layers.

\section{SpaGCN, stLearn, BayesSpace, Giotto.}

442 We ran these methods with the recommended parameters and set each one to generate the 443 same number of clusters as the ground truth layers. The stLearn-derived low-dimensional 444 embedding was used for downstream UMAP visualization and Harmony batch correction.

\section{Evaluation metrics for clustering.}

446 For datasets with cell-type labels (e.g., DLPFC), we employed ARI to compare the 447 performance of different clustering algorithms. ARI calculates the similarity between the 448 clustering labels predicted by the algorithm and reference cluster labels as:

$$
A R I=\frac{R I-E[R I]}{\max (R I)-E[R I]},
$$

449 where the unadjusted rand index $(\mathrm{RI})$ is defined as $R I=(a+b) / C_{n}^{2}$, with $a$ being the number

450 of pairs correctly labeled as coming from the same set, $b$ being the number of pairs correctly 451 labeled as not in the same set, and $C_{n}^{2}$ being the total number of possible pairs. $E[R I]$ is the 452 expected $R I$ of random labeling. A higher ARI score indicates better performance.

\section{Monocle3.}

454 On the DLPFC \#151673 slice and breast cancer data, we ran Monocle3 using both the 455 Seurat and SEDR outputs. For Seurat, we ran the standard pipeline to obtain the UMAP. For 456 SEDR, we first extracted the low-dimensional embedding and then used the uwot package to 
457 calculate the UMAP. We then ran Monocle3 on both UMAPs using the recommended

458 parameters and set white matter (WM) as the starting point to generate the pseudo-time.

459 Finally, we used the Moran_I test to detect significant genes that showed correlations with

460 the pseudo-time.

461 Leiden clustering, PAGA trajectory, and UMAPs.

462 We used the Scanpy (v.1.5.0) package to compute the Leiden clustering, partition-based 463 graph abstraction (PAGA), and uniform manifold approximation and projection (UMAP) from 464 SEDR-derived joint embeddings of gene expression and spatial information. Briefly, we used 465 SEDR embeddings to compute neighborhood graphs with 15 as the number of neighbors 466 and Euclidean distance as the distance measure. To obtain the same number of unique 467 Leiden clusters, grid-searching on the Leiden clustering resolutions between 0.2 and 2.5 was 468 performed at intervals of $0.05 / 0.01$. Subsequently, PAGA was applied to quantify the 469 connectivity between Leiden clusters. Finally, the cluster positions suggested by PAGA were 470 used to compute the UMAP for visualization.

Harmony.

472 Harmony was used to correct batch effects on low-dimensional embeddings. For SEDR, we 473 used latent space embeddings as input. For the raw data and stLearn, we used the PCA 474 embeddings. We treated different samples as different batches, and set all other parameters 475 to their default values. For each method, the uncorrected embeddings and batch-corrected 476 Harmony embeddings were used for UMAP visualization and analysis.

\section{Prediction of cell type composition of $10 x$ Visium spatial spot.}

478 We downloaded a published scRNA-seq dataset of human breast cancer ${ }^{36}$ as reference, and 479 ran Seurat to find transfer anchors between the reference and our Visium spatial data. Cell 480 types in the reference were then assigned to the spatial spots by label transferring. We 481 removed cell types with probabilities equal to 0 for all spots.

482 Differential expression analysis and pathway analyses. 
483 We used Seurat to identify DEGs. Genes with adjusted $p$-values $<0.05$ were used as the

484 input for QIANGEN Ingenuity Pathway Analysis (IPA). For IPA results, pathways with

485 positive or negative z-scores were plotted.

\section{Raw data processing of Stereo-seq data.}

487 Fastq files were generated using the MGI DNBSEQ-Tx sequencer. Coordinate identities 488 (CIDs) and unique molecular identifiers (UMIs) were encoded in the forward reads (CID: 1489 25bp, UMI: 26-35bp), while the reverse reads consisted of the cDNA sequences. CID 490 sequences in the forward reads were first mapped to the designed coordinates of the in situ 491 captured chip, allowing one base mismatch to correct for sequencing and PCR errors. Reads 492 with UMls containing either $\mathrm{N}$ bases or more than two bases with quality scores lower than 49310 were filtered out. The CIDs and UMls associated with each read were appended to each 494 read header. Retained reads were then aligned to the reference genome $(\mathrm{mm} 10)$ using $495 \mathrm{STAR}^{51}$, and mapped reads with $\mathrm{MAPQ} \geq 10$ were counted and annotated using an in-house 496 script (available at https://github.com/BGIResearch/handleBam). UMls with the same CIDs 497 and gene loci were collapsed together, allowing for one mismatch to correct for sequencing 498 and PCR errors, to give the final gene expression matrix.

Local inverse Simpson's index (LISI).

We first used Seurat and SEDR to generate cell clusters for the stereo-seq data, and then the R "lisi" package to calculate the LISIs using spatial coordinates as X and the clustering results of Seurat and SEDR as meta data.

Data availability.

505 (1) LIBD human dorsolateral prefrontal cortex (DLPFC) Data 506 (http://spatial.libd.org/spatialLIBD/); (2) 10x visium spatial transcriptomics data of human 507 breast cancer and Stereo-seq of mouse olfactory bulb are at 508 https://github.com/JinmiaoChenLab/SEDR/ (3) Analysis results and scripts to reproduce the 
bioRxiv preprint doi: https://doi.org/10.1101/2021.06.15.448542; this version posted July 2, 2021. The copyright holder for this preprint (which was not certified by peer review) is the author/funder, who has granted bioRxiv a license to display the preprint in perpetuity. It is made available under aCC-BY-NC-ND 4.0 International license.

509 results are at https://github.com/JinmiaoChenLab/SEDR/

510

511 Software availability.

512 SEDR was written in Python using the PyTorch library. An open-source implementation of

513 SEDR has been released on https://github.com/HzFu/SEDR

514

515

516

517 


\section{References}

519 1. Ståhl, P. L. et al. Visualization and analysis of gene expression in tissue sections by $520 \quad$ spatial transcriptomics. Science (2016) doi:10.1126/science.aaf2403.

521 2. Maynard, K. R. et al. Transcriptome-scale spatial gene expression in the human 522 dorsolateral prefrontal cortex. Nat. Neurosci. 24, 425-436 (2021).

523 3. Ji, A. L. et al. Multimodal Analysis of Composition and Spatial Architecture in Human 524 Squamous Cell Carcinoma. Cell (2020) doi:10.1016/j.cell.2020.08.043.

525 4. Rodriques, S. G. et al. Slide-seq: A scalable technology for measuring genome-wide expression at high spatial resolution. Science (80-. ). (2019)

5. Chen, A. et al. Large field of view-spatially resolved transcriptomics at nanoscale resolution Short title: DNA nanoball stereo-sequencing. bioRxiv 2021.01.17.427004 (2021).

531 6. Fu, X. et al. Continuous Polony Gels for Tissue Mapping with High Resolution and RNA Capture Efficiency. bioRxiv 2021.03.17.435795 (2021).

533 7. Cho, C.-S. et al. Seq-Scope: Submicrometer-resolution spatial transcriptomics for $534 \quad$ single cell and subcellular studies. bioRxiv (2021).

535 8. Stickels, R. R. et al. Highly sensitive spatial transcriptomics at near-cellular resolution with Slide-seqV2. Nat. Biotechnol. (2021) doi:10.1038/s41587-020-0739-1.

537 9. Liu, Y. et al. High-Spatial-Resolution Multi-Omics Sequencing via Deterministic Barcoding in Tissue. Cell (2020) doi:10.1016/j.cell.2020.10.026.

539 10. Lein, E., Borm, L. E. \& Linnarsson, S. The promise of spatial transcriptomics for $540 \quad$ neuroscience in the era of molecular cell typing. Science (2017) doi:10.1126/science.aan6827.

542 11. Yoosuf, N., Navarro, J. F., Salmén, F., Ståhl, P. L. \& Daub, C. O. Identification and transfer of spatial transcriptomics signatures for cancer diagnosis. Breast Cancer Res. 
$544 \quad$ (2020) doi:10.1186/s13058-019-1242-9.

545 12. van den Brink, S. C. et al. Single-cell and spatial transcriptomics reveal somitogenesis

546 in gastruloids. Nature (2020) doi:10.1038/s41586-020-2024-3.

547 13. Dong, R. \& Yuan, G. C. SpatialDWLS: accurate deconvolution of spatial

$548 \quad$ transcriptomic data. Genome Biol. 22, 1-10 (2021).

549 14. Song, Q. \& Su, J. DSTG: deconvoluting spatial transcriptomics data through graph-

$550 \quad$ based artificial intelligence. Brief. Bioinform. (2021) doi:10.1093/bib/bbaa414.

15. Andersson, A. et al. Single-cell and spatial transcriptomics enables probabilistic inference of cell type topography. Commun. Biol. 3, 1-8 (2020).

16. Biancalani, T. et al. Deep learning and alignment of spatially-resolved whole transcriptomes of single cells in the mouse brain with Tangram. bioRxiv (2020).

17. Gayoso, A. et al. scvi-tools: a library for deep probabilistic analysis of single-cell omics data. bioRxiv (2021).

18. Lopez, R. et al. Multi-resolution deconvolution of spatial transcriptomics data reveals continuous patterns of inflammation. bioRxiv (2021).

19. Elosua-Bayes, M., Nieto, P., Mereu, E., Gut, I. \& Heyn, H. SPOTlight: seeded NMF regression to deconvolute spatial transcriptomics spots with single-cell transcriptomes. Nucleic Acids Res. (2021) doi:10.1093/nar/gkab043.

20. Danaher, P. et al. Advances in mixed cell deconvolution enable quantification of cell types in spatially-resolved gene expression data. bioRxiv (2020).

21. Hao, Y. et al. Integrated analysis of multimodal single-cell data. Bioarxiv (2020).

22. Zhao, E. et al. Bayesspace enables the robust characterization of spatial gene expression architecture in tissue sections at increased resolution. bioRxiv (2020) doi:10.1101/2020.09.04.283812.

23. Dries, R. et al. Giotto: a toolbox for integrative analysis and visualization of spatial expression data. Genome Biol. 22, 78 (2021).

24. Hu, J. et al. Integrating gene expression, spatial location and histology to identify spatial 1 domains and spatially variable genes by graph convolutional network 23 . 
bioRxiv 2020.11.30.405118 (2020).

25. Pham, D. et al. stLearn: Integrating spatial location, tissue morphology and gene expression to find cell types, cell-cell interactions and spatial trajectories within undissociated tissues. bioRxiv (2020) doi:10.1101/2020.05.31.125658.

26. Xie, J., Girshick, R. \& Farhadi, A. Unsupervised Deep Embedding for Clustering Analysis. in ICML (eds. Balcan, M. F. \& Weinberger, K. Q.) vol. 48 478-487 (PMLR, 2016).

27. Mann, H. B. \& Whitney, D. R. On a Test of Whether one of Two Random Variables is Stochastically Larger than the Other. Ann. Math. Stat. 18, 50-60 (1947).

28. Trapnell, C. et al. The dynamics and regulators of cell fate decisions are revealed by pseudotemporal ordering of single cells. Nat. Biotechnol. 32, 381-386 (2014).

29. Cao, J. et al. The single-cell transcriptional landscape of mammalian organogenesis. Nature 566, 496-502 (2019).

30. Wolf, F. A. et al. PAGA: graph abstraction reconciles clustering with trajectory inference through a topology preserving map of single cells. Genome Biol. 20, 1-9 (2019).

31. Gilmore, E. G. \& Herrup, K. Cortical development: Layers of complexity. Current Biology (1997) doi:10.1016/s0960-9822(06)00108-4.

32. Chini, M. \& Hanganu-Opatz, I. L. Prefrontal Cortex Development in Health and Disease: Lessons from Rodents and Humans. Trends in Neurosciences (2021) doi:10.1016/j.tins.2020.10.017.

33. Nadarajah, B. \& Parnavelas, J. G. Modes of neuronal migration in the developing cerebral cortex. Nature Reviews Neuroscience (2002) doi:10.1038/nrn845.

34. Tran, H. T. N. et al. A benchmark of batch-effect correction methods for single-cell RNA sequencing data. Genome Biol. (2020) doi:10.1186/s13059-019-1850-9.

35. Nguyen, P. H. D. et al. Intratumoural immune heterogeneity as a hallmark of tumour evolution and progression in hepatocellular carcinoma. Nat. Commun. 12, 1-13 (2021). 
600 36. et al. A single-cell RNA expression atlas of normal, preneoplastic and tumorigenic states in the human breast. EMBO J. 40, 1-23 (2021).

602 37. Friedl, P. \& Alexander, S. Cancer invasion and the microenvironment: Plasticity and reciprocity. Cell (2011) doi:10.1016/j.cell.2011.11.016.

38. Kuroda, H. et al. Tumor microenvironment in triple-negative breast cancer: the

39. Asiry, S. et al. The Cancer Cell Dissemination Machinery as an Immunosuppressive Niche: A New Obstacle Towards the Era of Cancer Immunotherapy. Front. Immunol. 12, 1-19 (2021).

41. Olson, O. C. \& Joyce, J. A. Cysteine cathepsin proteases: Regulators of cancer progression and therapeutic response. Nature Reviews Cancer (2015) doi:10.1038/nrc4027.

42. Roumenina, L. T. et al. Tumor cells hijack macrophage-produced complement C1q to promote tumor growth. Cancer Immunol. Res. (2019) doi:10.1158/2326-6066.CIR-180891.

43. Fraser, D., Melzer, E., Camacho, A. \& Gomez, M. Macrophage production of innate immune protein $\mathrm{C} 1 \mathrm{q}$ is associated with M2 polarization (INM1P.434). J. Immunol. (2015).

44. Monteran, L. \& Erez, N. The dark side of fibroblasts: Cancer-associated fibroblasts as mediators of immunosuppression in the tumor microenvironment. Frontiers in Immunology (2019) doi:10.3389/fimmu.2019.01835.

45. Lin, Y., Xu, J. \& Lan, H. Tumor-associated macrophages in tumor metastasis: Biological roles and clinical therapeutic applications. Journal of Hematology and Oncology (2019) doi:10.1186/s13045-019-0760-3. 
628 46. Deng, Y. et al. Resource High-Spatial-Resolution Multi-Omics Sequencing via

629 Deterministic Barcoding in Tissue II II Resource High-Spatial-Resolution Multi-Omics

630 Sequencing via Deterministic Barcoding in Tissue. Cell 183, 1665-1681.e18 (2020).

631 47. Weinreb, C., Wolock, S. \& Klein, A. M. SPRING: a kinetic interface for visualizing high dimensional single-cell expression data. Bioinformatics 34, 1246-1248 (2018).

633 48. Kipf, T. N. \& Welling, M. Variational Graph Auto-Encoders. in NIPS Workshop on Bayesian Deep Learning (2016).

49. Kipf, T. N. \& Welling, M. Semi-Supervised Classification with Graph Convolutional Networks. in International Conference on Learning Representations (ICLR) (2017).

50. Hafemeister, C. \& Satija, R. Normalization and variance stabilization of single-cell RNA-seq data using regularized negative binomial regression. Genome Biol. 20, 296 (2019).

640 51. Dobin, A. et al. STAR: Ultrafast universal RNA-seq aligner. Bioinformatics (2013) doi:10.1093/bioinformatics/bts635. 


\section{Acknowledgements:}

645 This research was supported by funding from Singapore Immunology Network (SIgN), A *

646 STAR, Singapore.

648 Author contributions:

649 Huazhu Fu designed and implemented SEDR. Hang Xu, Huazhu Fu, Kelvin Chong,

650 Mengwei Li, Hong Kai Lee and Jingjing Ling performed data analysis. Hang Xu, Huazhu Fu, 651 Mengwei Li generated figures. Jinmiao Chen, Huazhu Fu, Hang Xu, Kok Siong Ang, Kelvin 652 Chong, Jingjing Ling and Ling Shao drafted the manuscript. Ao Chen and Longqi Liu provided Stereo-seq data. Jinmiao Chen conceptualized and supervised the study.

\section{Competing interests:}

656 The authors declare no competing interests.

\section{Figure legends:}

659 Figure 1. Overview of SEDR. SEDR learns a low-dimensional latent representation of gene 660 expression embedded with spatial information by jointly training a deep autoencoder and a 661 variational graph autoencoder. The low-dimensional embedding produced by SEDR can be 662 used for downstream visualization, cell clustering, trajectory inference, and batch effect correction.

\section{Figure 2. Quantitative assessment of SEDR on the human dorsolateral prefrontal} cortex (DLPFC) dataset. A) Ground-truth segmentation of cortical layers; clustering results of Seurat, Giotto, stLearn, SpaGCN, BayesSpace, and SEDR on DLPFC slice \#151673; and adjusted rand index (ARI) of various cluster sets on the 12 DLPFC slices. B) UMAP visualization and Monocle3 trajectory generated using the Seurat-derived PCA embedding 669 (left) and SEDR embedding (right). Monocle pseudotimes visualized on UMAP plots (middle) 670 and spatial co-ordinates (bottom). C) PAGA graphs generated using the Seurat-derived PCA 
671 embedding (top) and SEDR embedding (middle). The ratios of the sum of weights of

672 correctly inferred PAGA edges to the sum of weights of all edges produced by SEDR and

673 Seurat (bottom).

674 Figure 3. Batch effects present in DLPFC dataset and assessment of SEDR's 675 performance on batch correction. A) Slices \#151507, \#151672 and \#151673 showed 676 substantial inter-slice variations before batch effect correction. UMAP plots colored by 677 ground-truth cortical layers (left), slices (right), split by layers and colored by slices (bottom).

678 B) Harmony alone was unable to remove the batch effects present. C) SEDR alone 679 substantially reduced the batch effects. D) SEDR combined with Harmony effectively 680 corrected the batch effects. E) stLearn combined with Harmony was unable to correct the 681 batch effects.

\section{Figure 4. Application of SEDR on 10x Visium spatial transcriptomics data of human}

breast cancer. A) Manual pathology labeling based on H\&E staining (annotation); clustering results of SEDR, Seurat, stLearn, SpaGCN, and BayesSpace. B) Seurat3 'anchor'-based integration workflow was used to perform probabilistic transfer of annotations from a reference scRNA-seq data of human breast cancer to the spatial data. This gives a probabilistic classification of the scRNA-seq derived classes for each spot. The probabilities of tumor associated macrophage (TAMs) and cycling epithelials (C-EPI) were visualized. C) Pathways enriched by genes differentially expressed between SEDR clusters 3 (core) and 7 690 (outer ring). Red bars represent pathways upregulated in cluster 3. D) Trajectory analysis 691 results using PAGA (top) and Monocle3 (bottom). The PAGA graph predictions of the inter692 relatedness between the manually annotated DCIS/LCIS and IDC regions. The edge width 693 denotes connectivity strength, thus indicating the likelihood of an actual connection being 694 present. Monocle3 inferred the pseudo-times of spots in SEDR clusters 3, 7, and 11 using 695 the Seurat-derived PCA embedding (termed "rna_pseudotime") and SEDR embedding 696 (termed "SEDR_pseudotime"). E) Heatmap of genes with expression changes along the 697 Monocle-derived pseudo-time. 
698 Figure 5. Application of SEDR on Stereo-seq spatial transcriptomics data of mouse

699 olfactory bulb tissue sections. A) Laminar organization of DAPI-stained mouse olfactory

700 bulb. B) Unsupervised clustering of the spatial voxels analyzed by Seurat and SEDR. C) The

701 four clusters with the highest numbers of voxels were selected and visualized. D)

702 Quantitative comparison of Seurat and SEDR clusters using local inverse Simpson's index

703 (LISI).

704 Supplementary:

705 Figure 1. Human breast cancer histology and cell type mixtures of spatial spots. A)

706 H\&E staining. B) Seurat3 predicted probabilities of scRNA-seq derived cell types.

707 Figure 2. Differentially expressed genes (DEGs) and enriched pathways. A) Locations of

708 DCIS_LCIS_3 and other DCIS_LCIS regions. B) Top DEGs between DCIS_LCIS_3 and

709 other DCIS_LCIS regions. C) Enriched pathways of DEGs between DCIS_LCIS_3 and other

710 DCIS_LCIS regions. Red bars represent pathways up-regulated in DCIS_LCIS_3 D)

711 Percentages of tumor associated macrophages (TAMs) in SEDR cluster 3 (tumor core) and

712 cluster 7 (tumor edge). E) Violin plots of selected DEGs between SEDR clusters 3 and 7. 
Adjacency matrix $A$

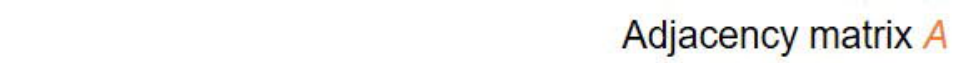




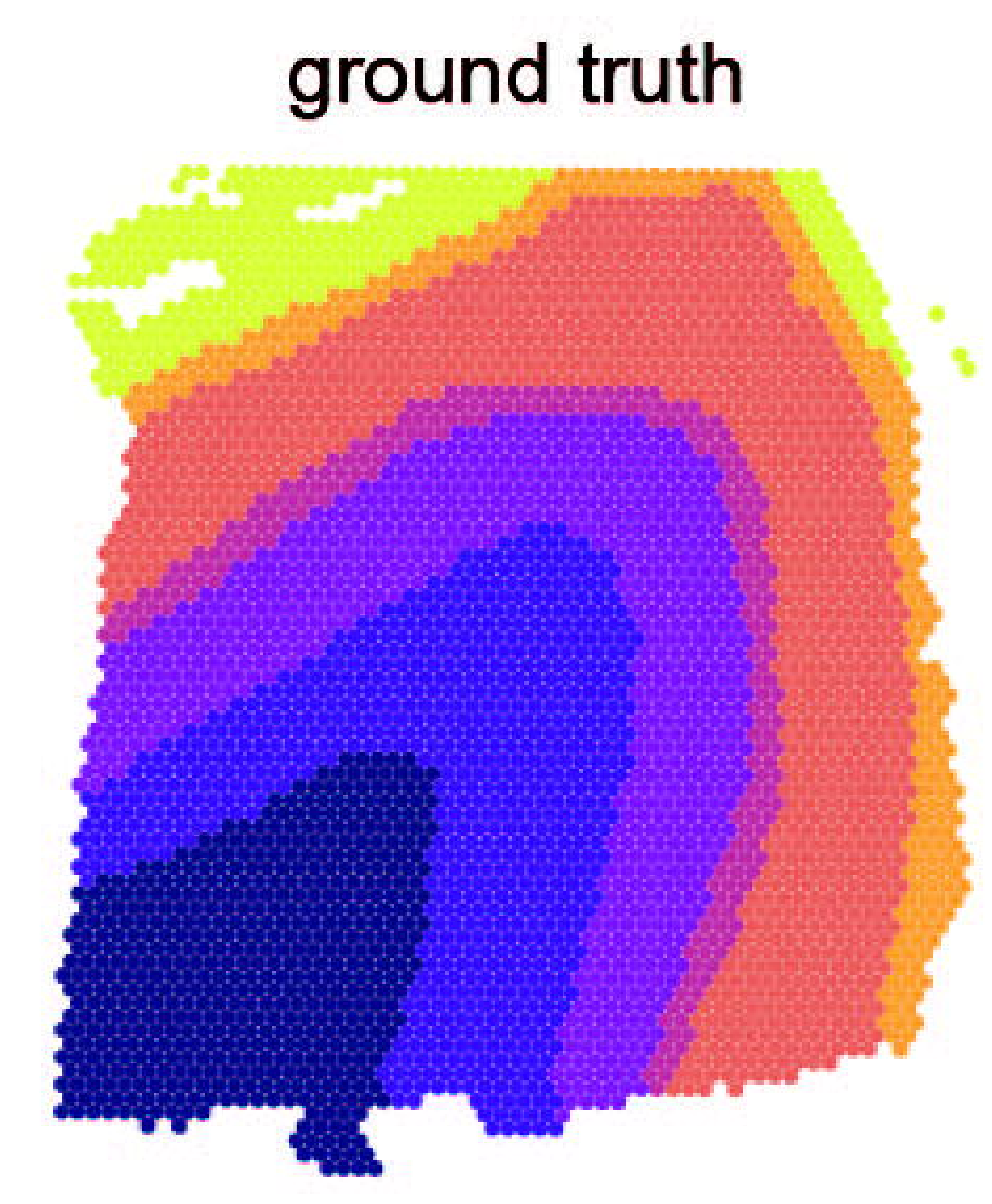

SpaGCN: $A R I=0.383$

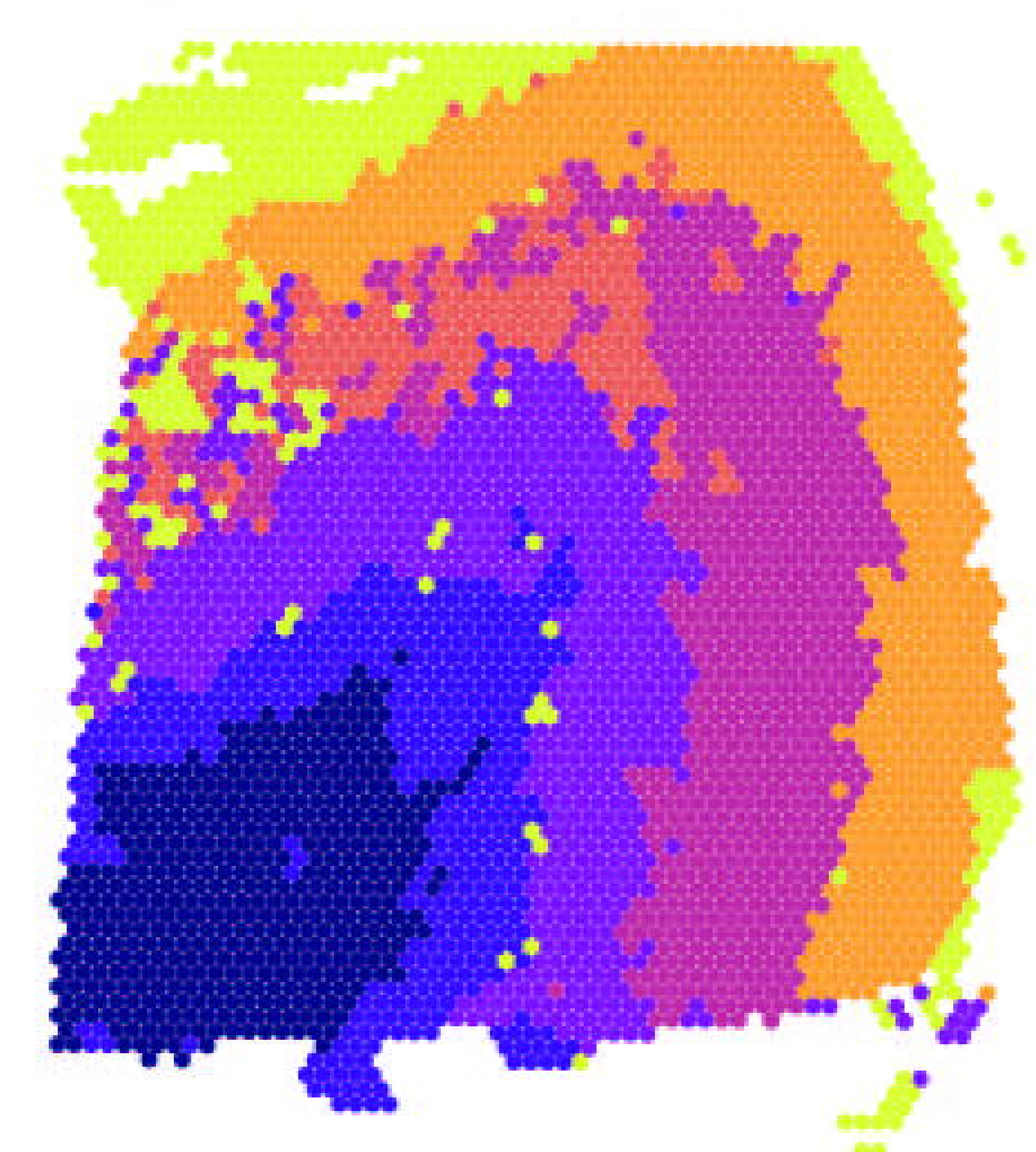

B

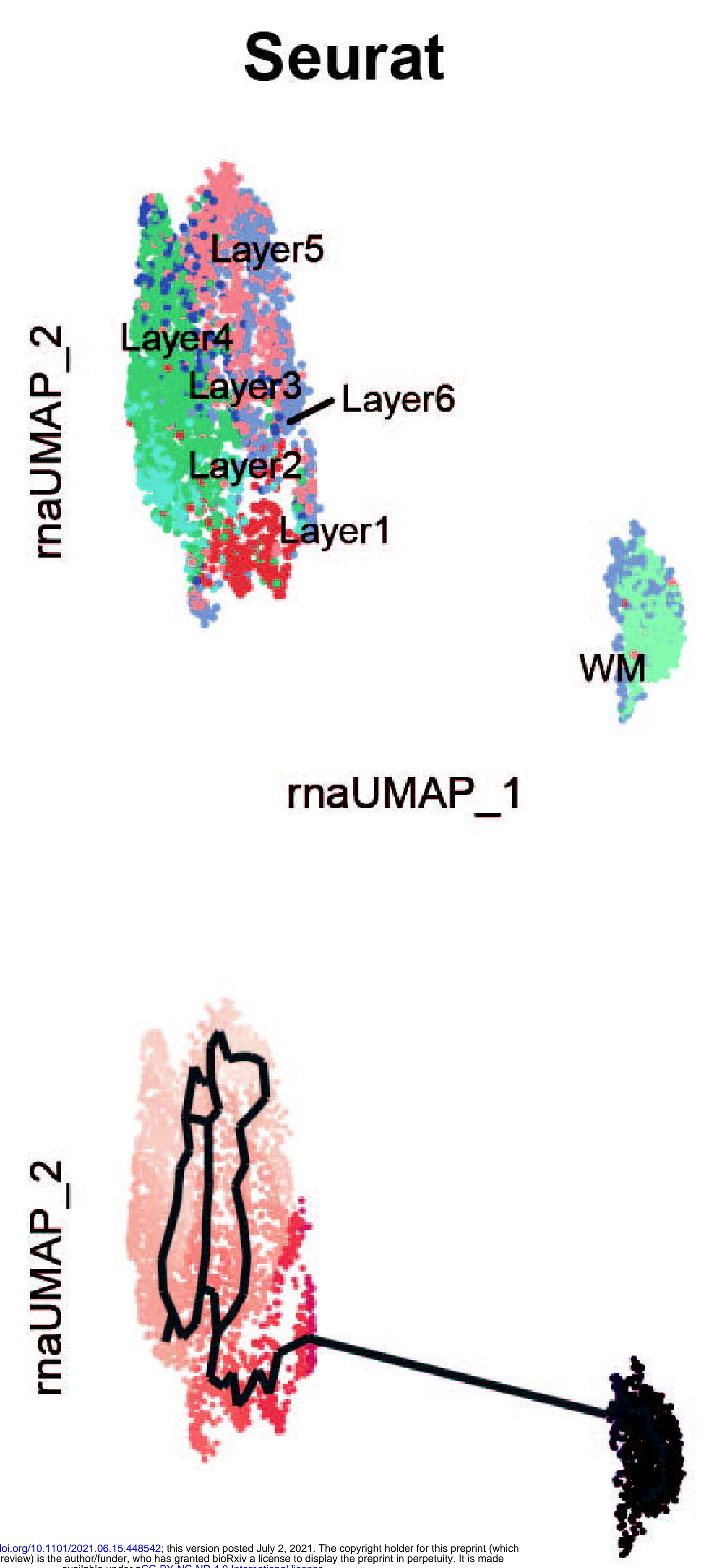

rnaUMAP_1

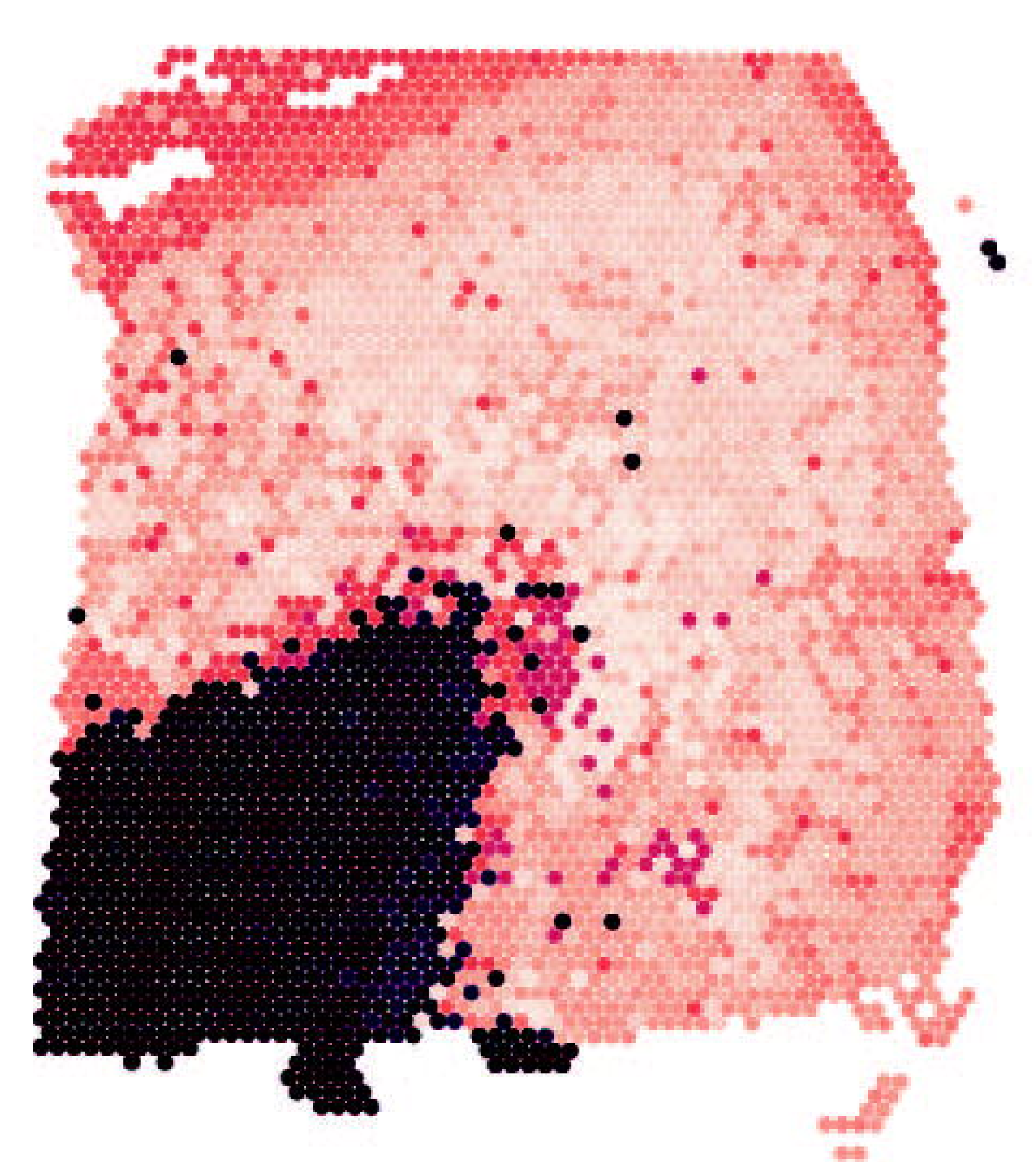

pseudotime
Seurat: $A R I=0.389$

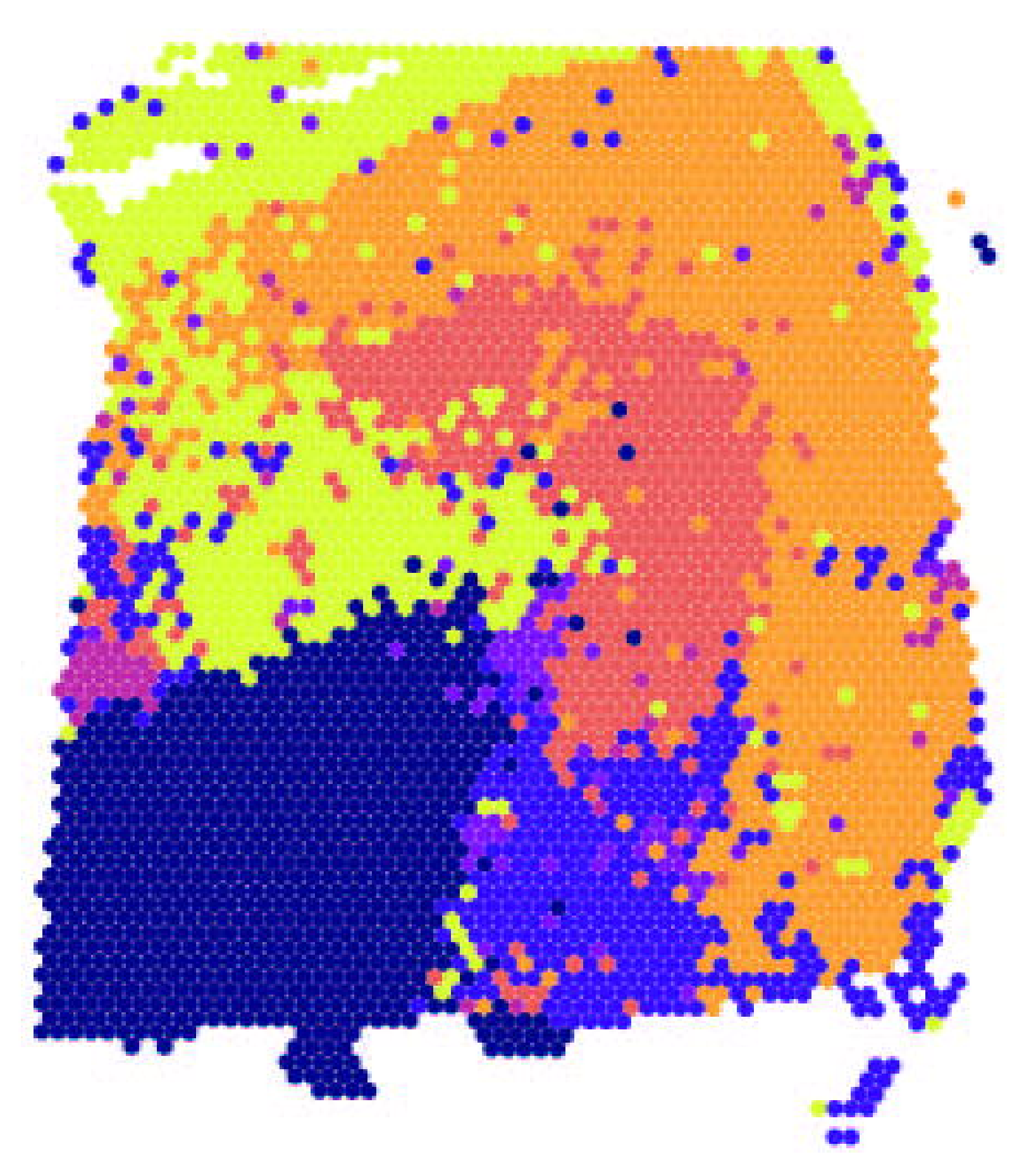

BayesSpace: $A R I=0.55$
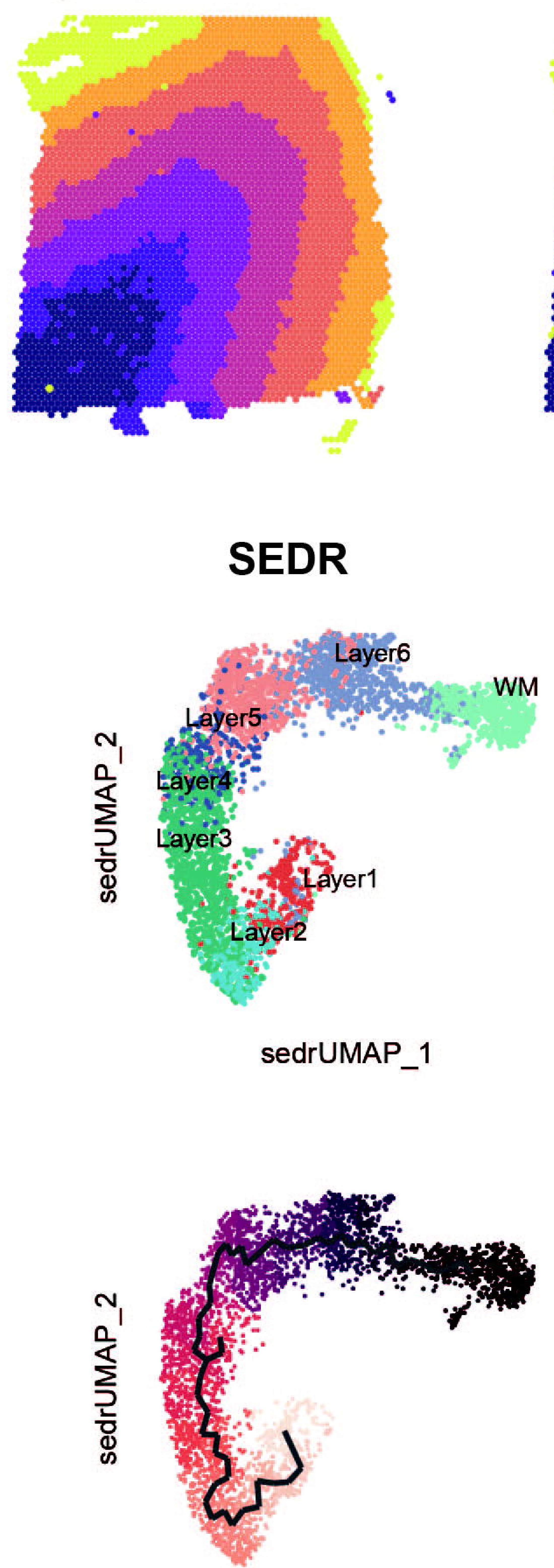

sedrUMAP_1

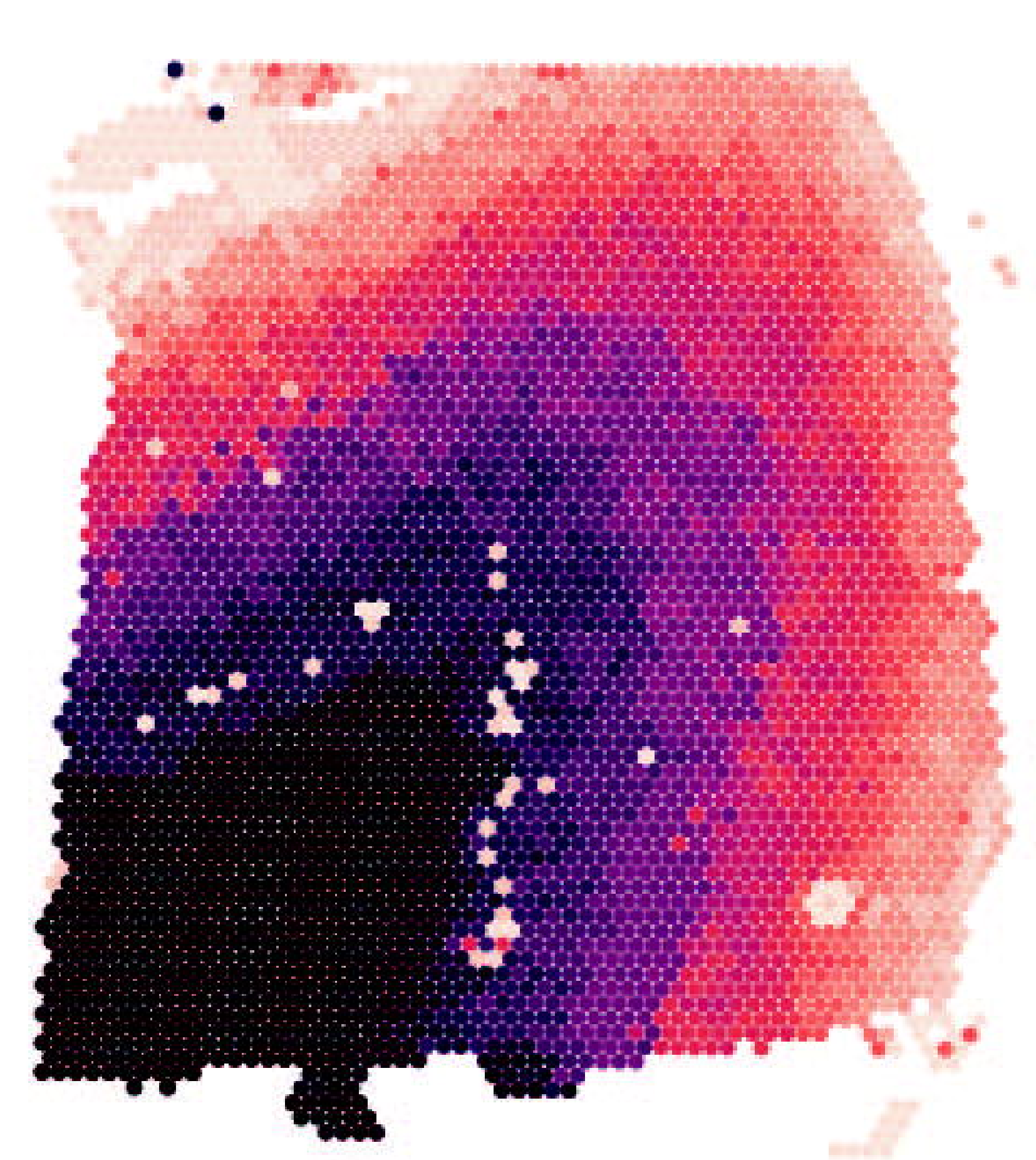

pseudotime
Giotto: $A R I=0.367$

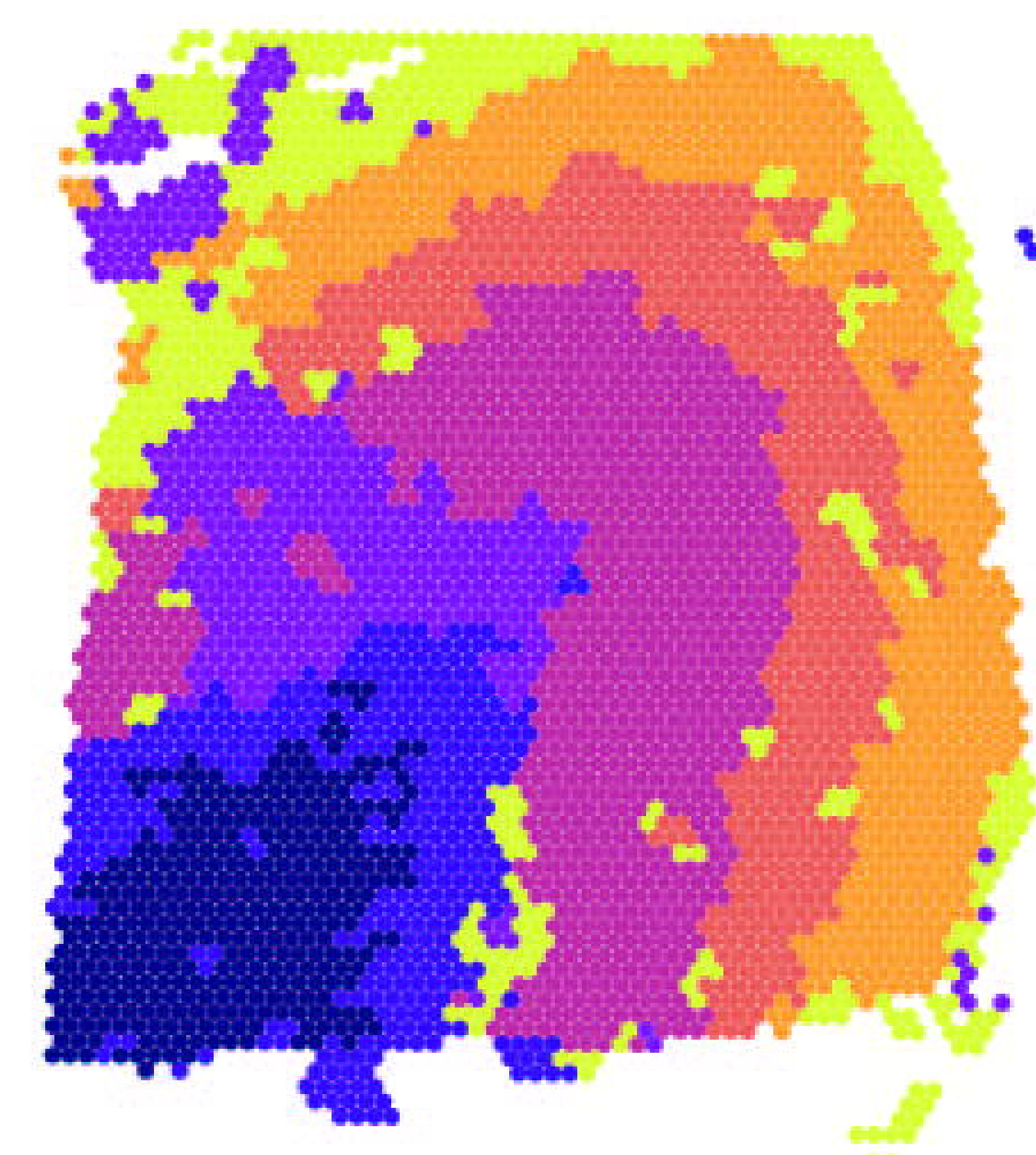

\section{SEDR: $A R I=0.573$}

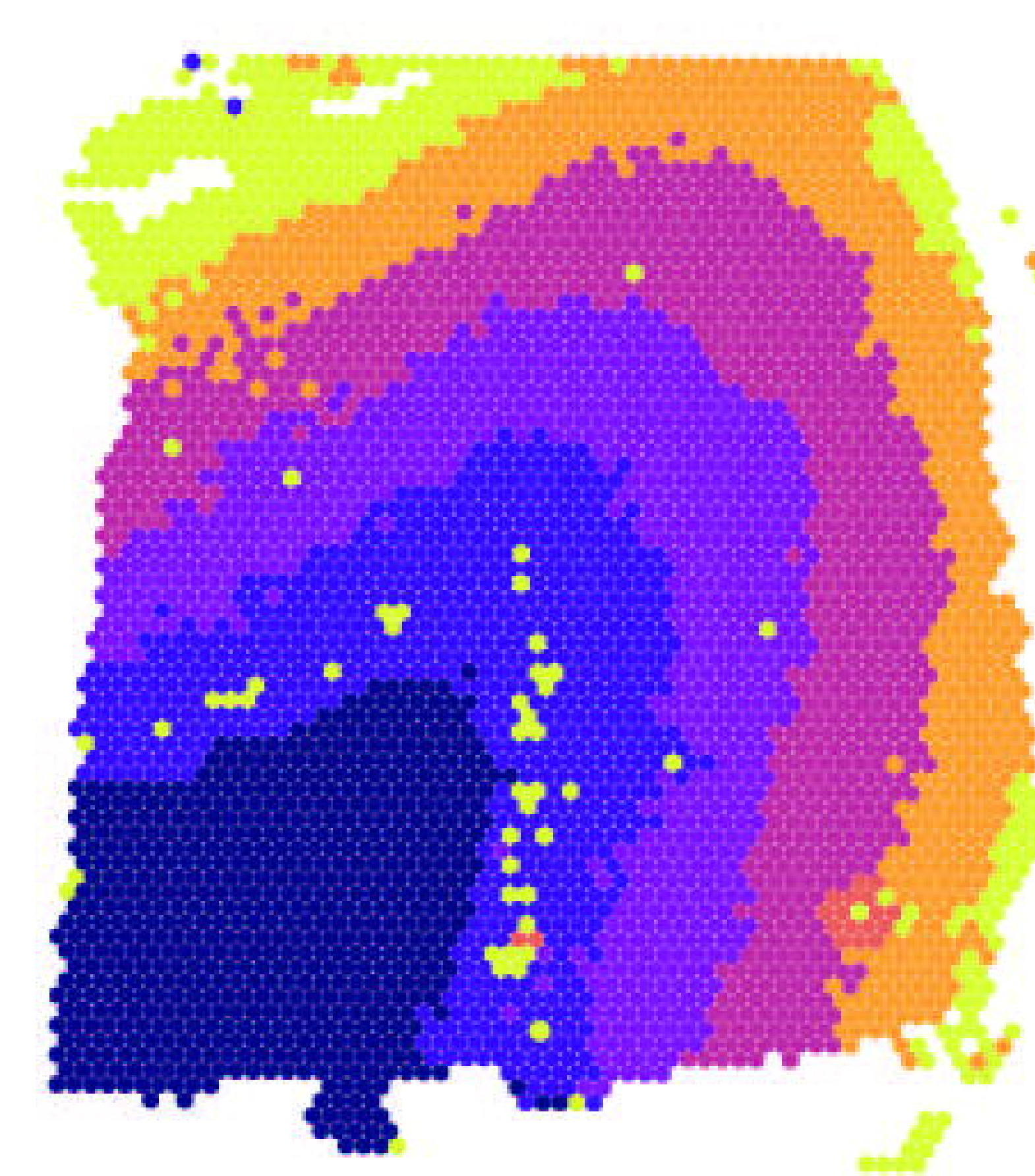

C
stLearn: $A R I=0.308$
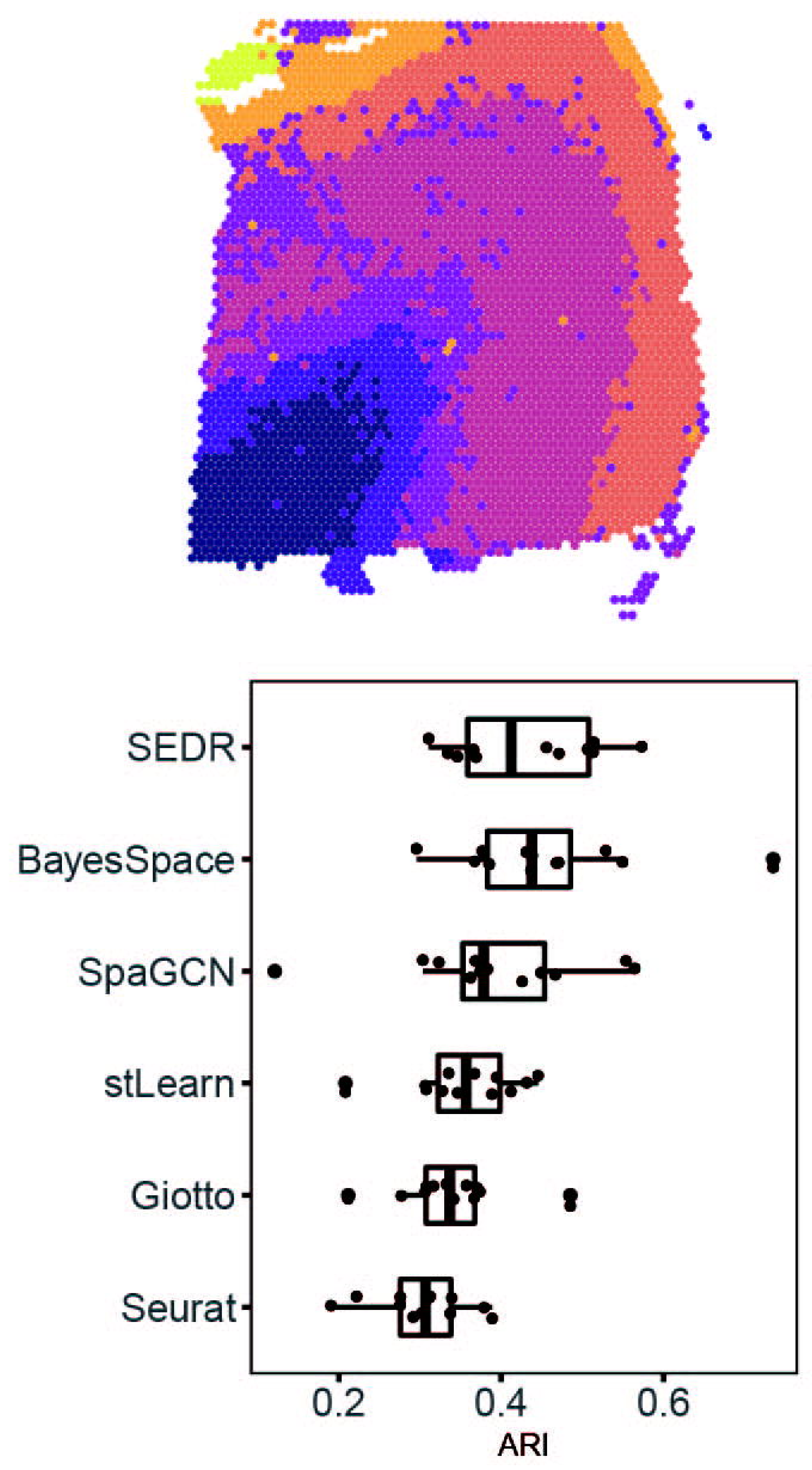

Seurat
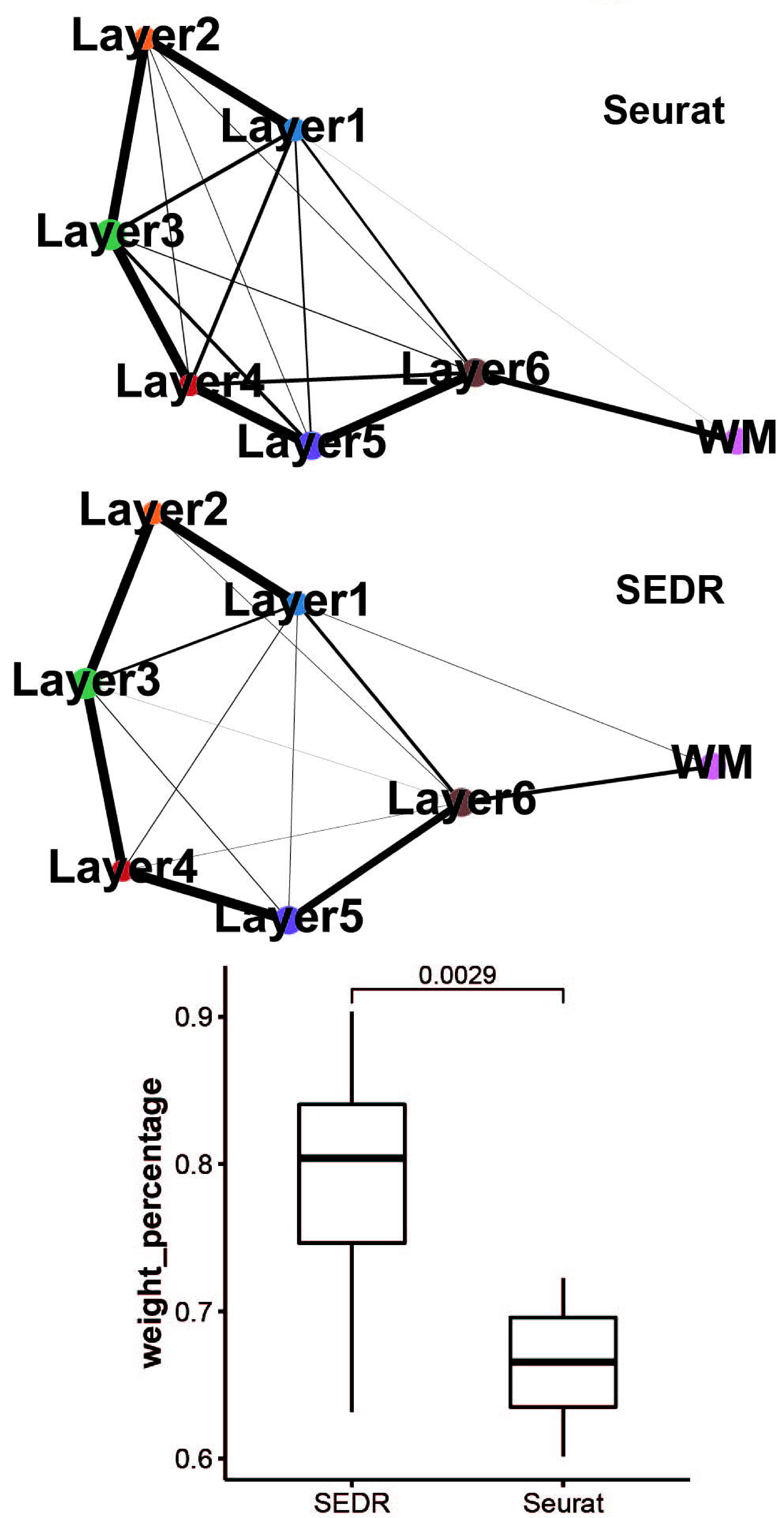


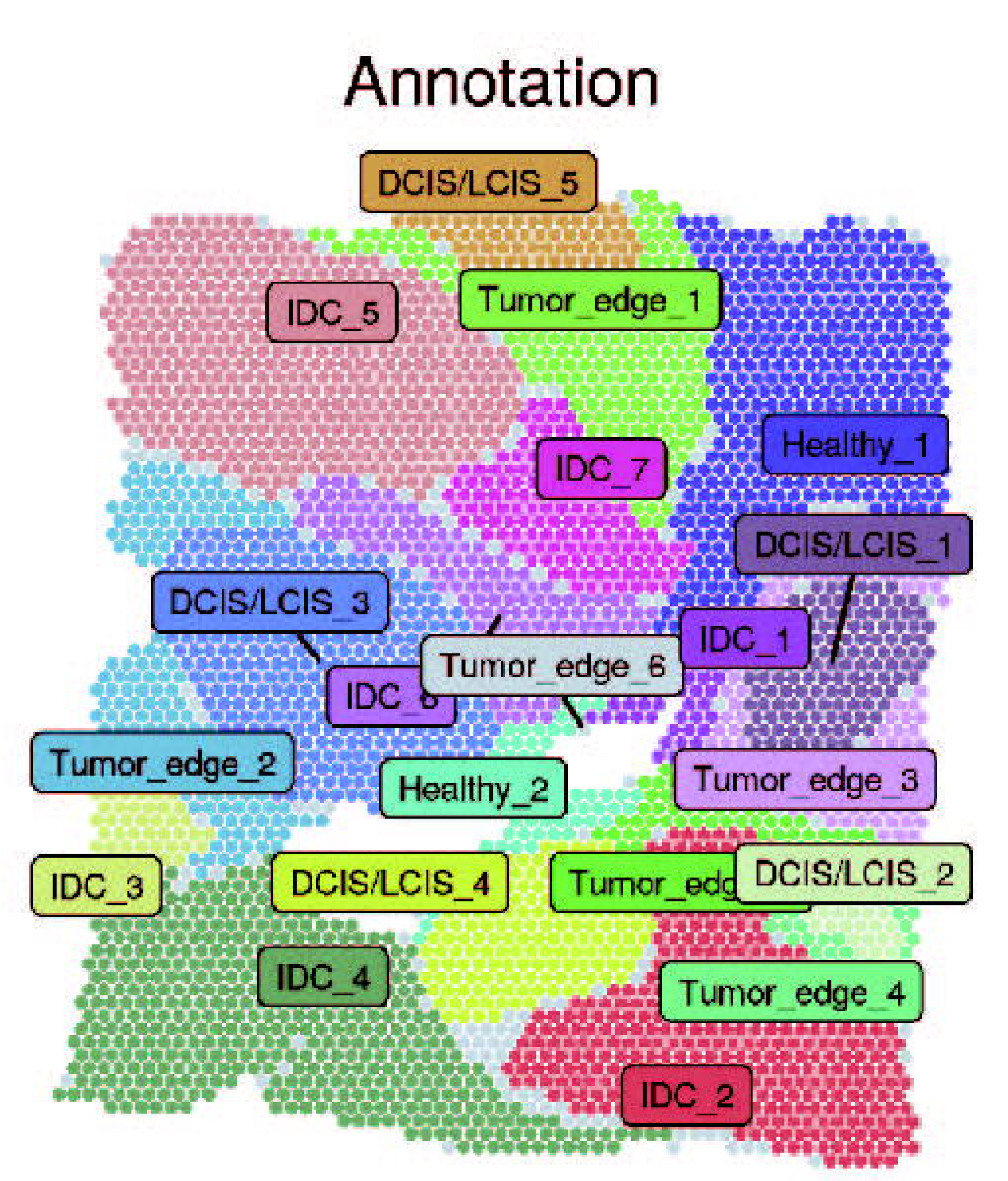

stLearn

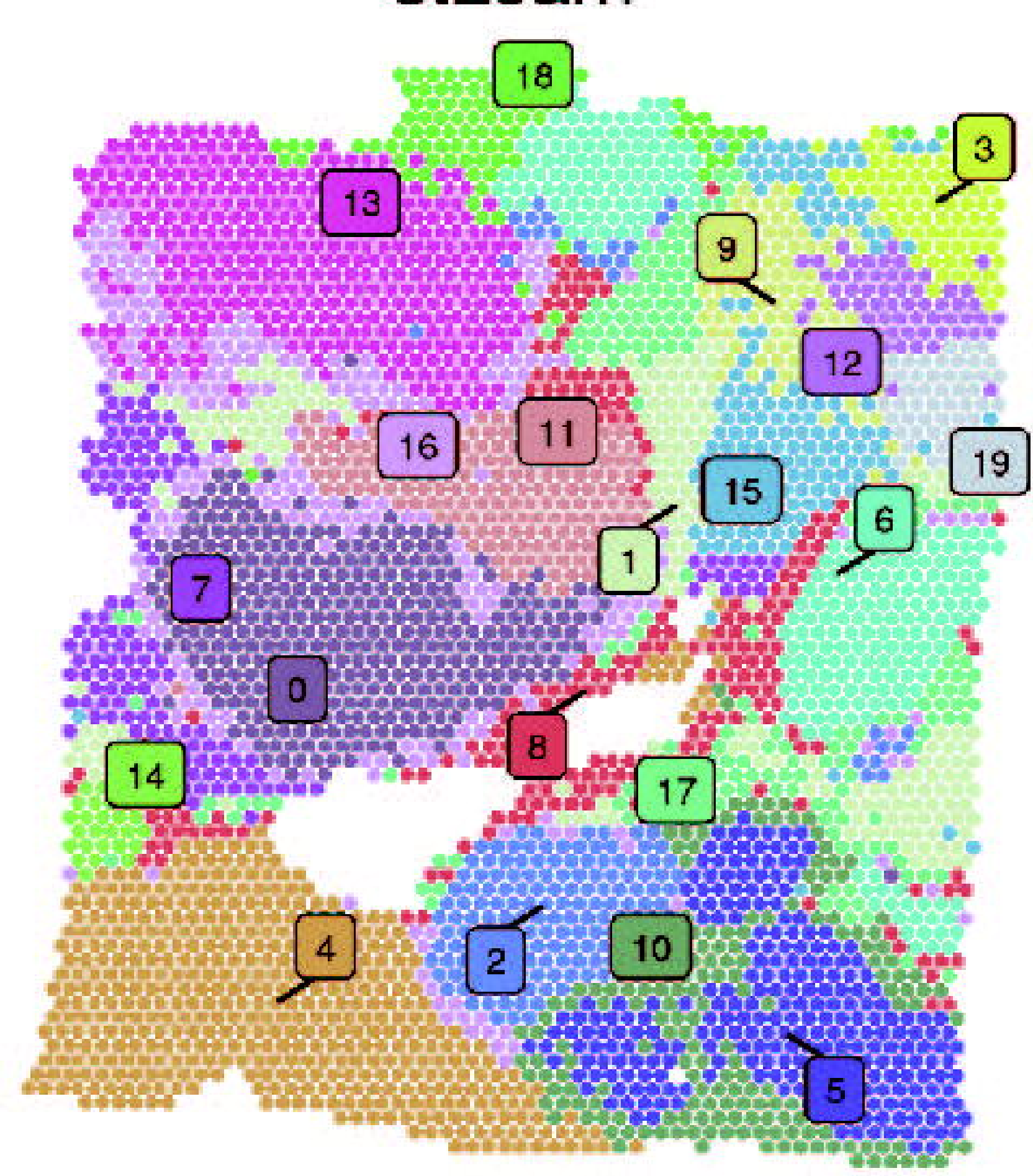

C

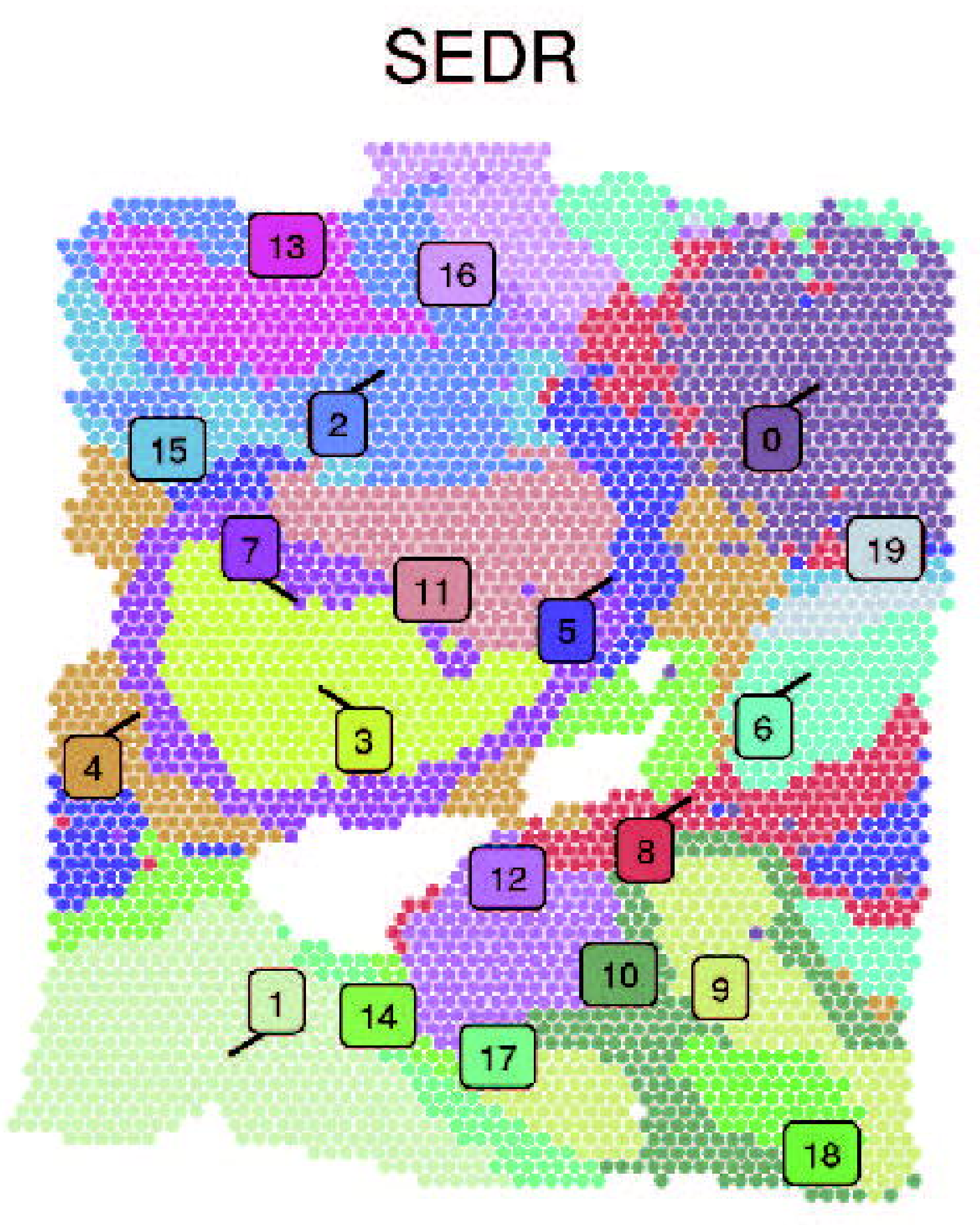

SpaGCN

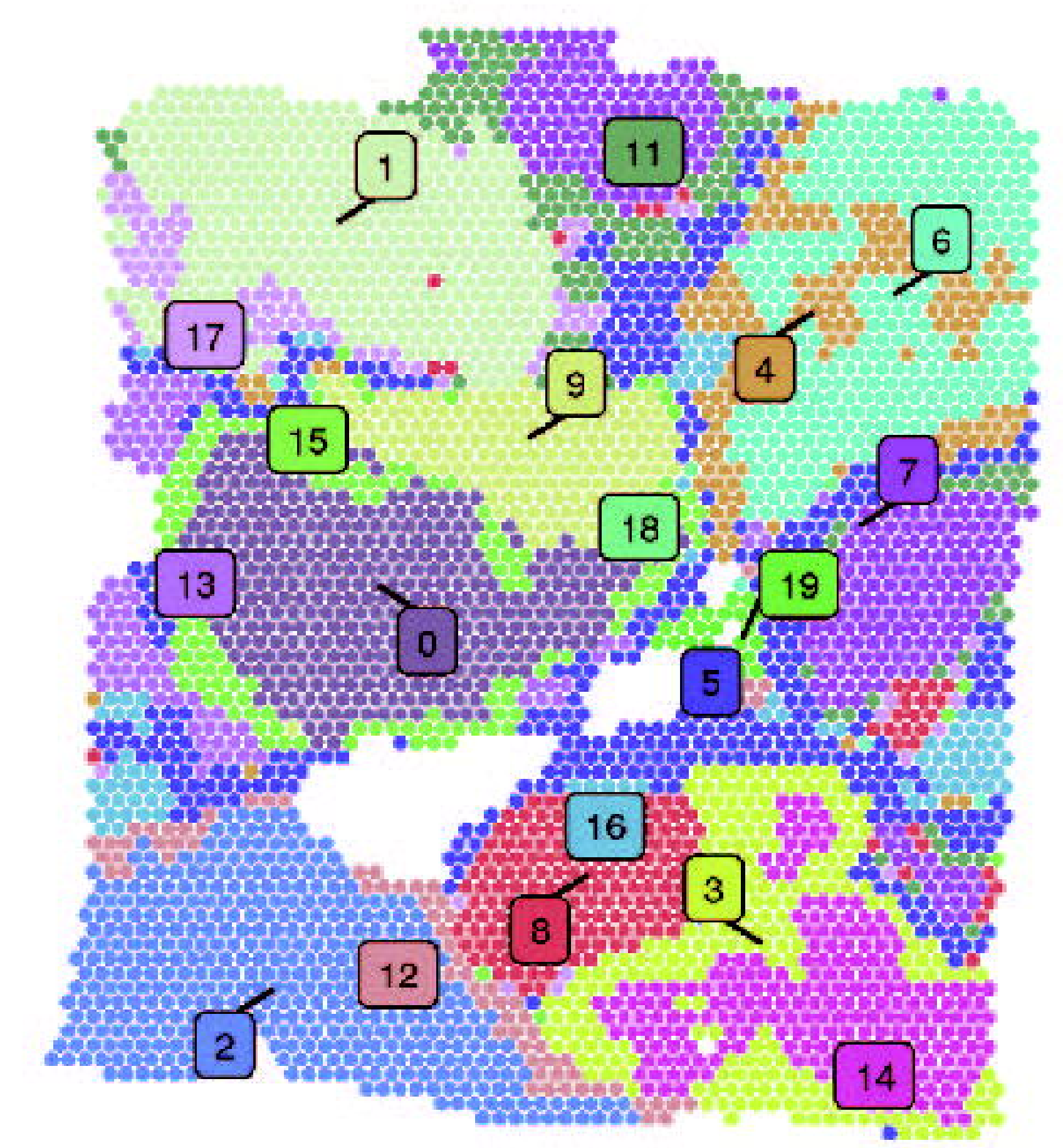

Seurat

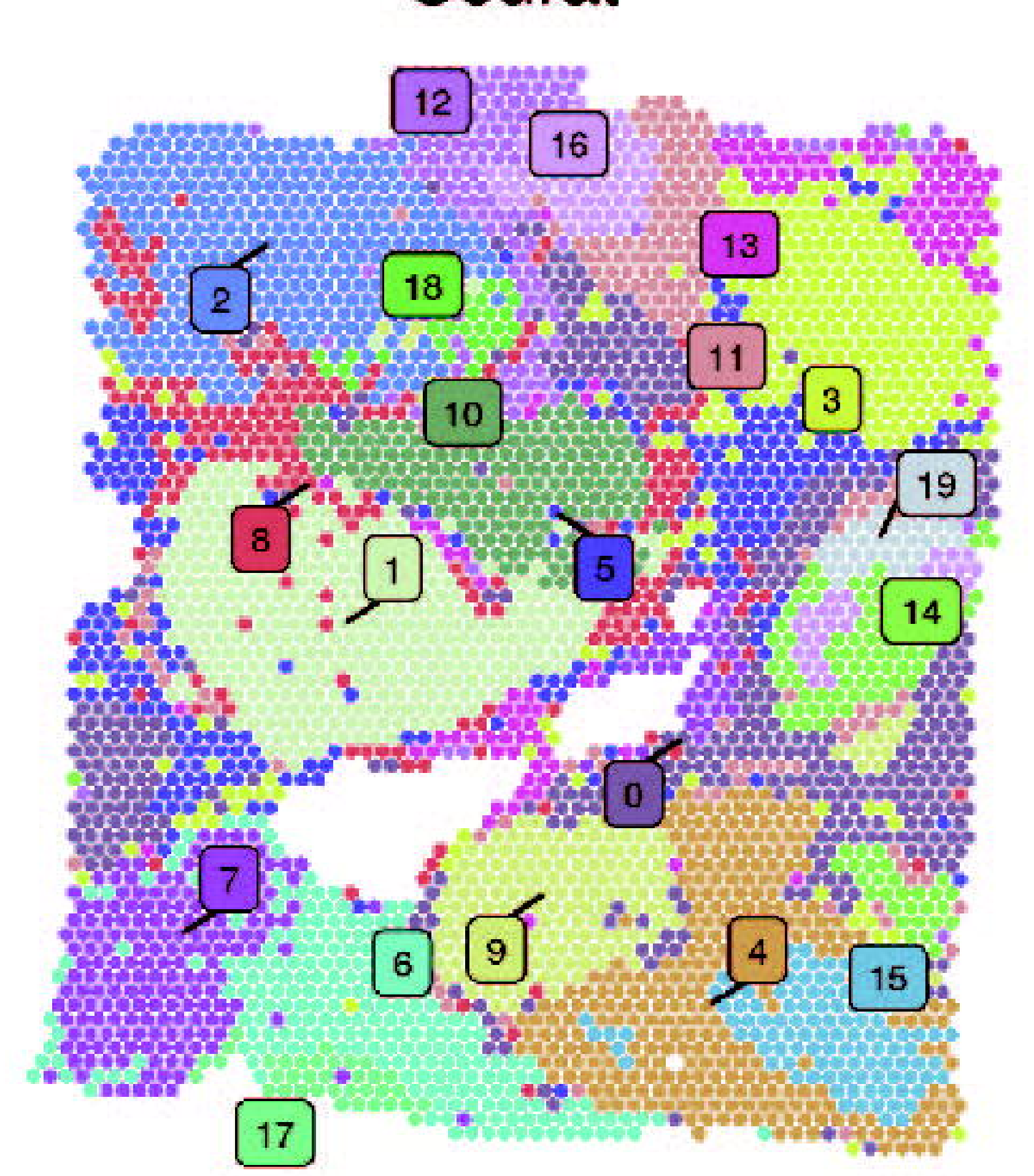

BayesSpace

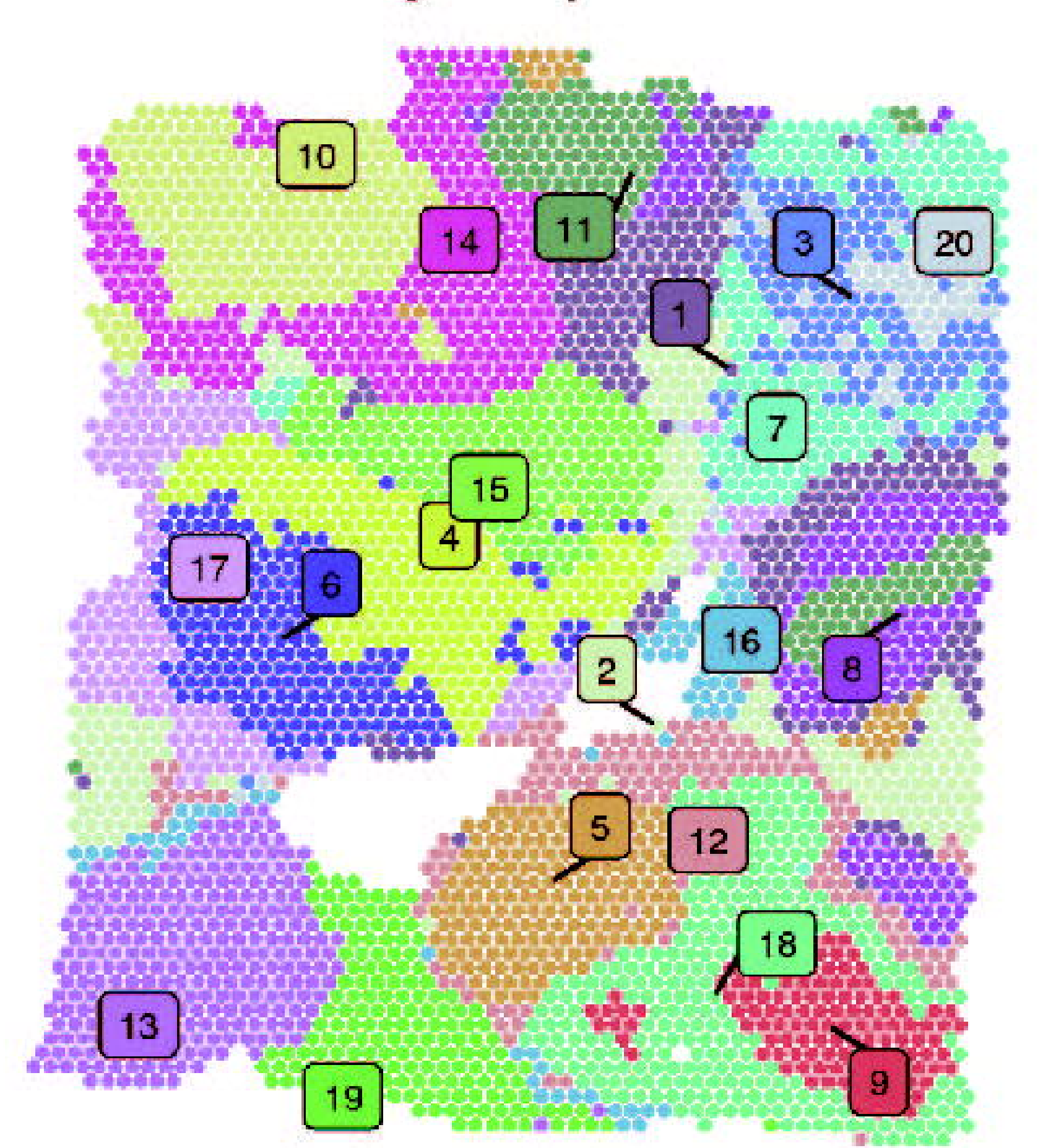

TAM

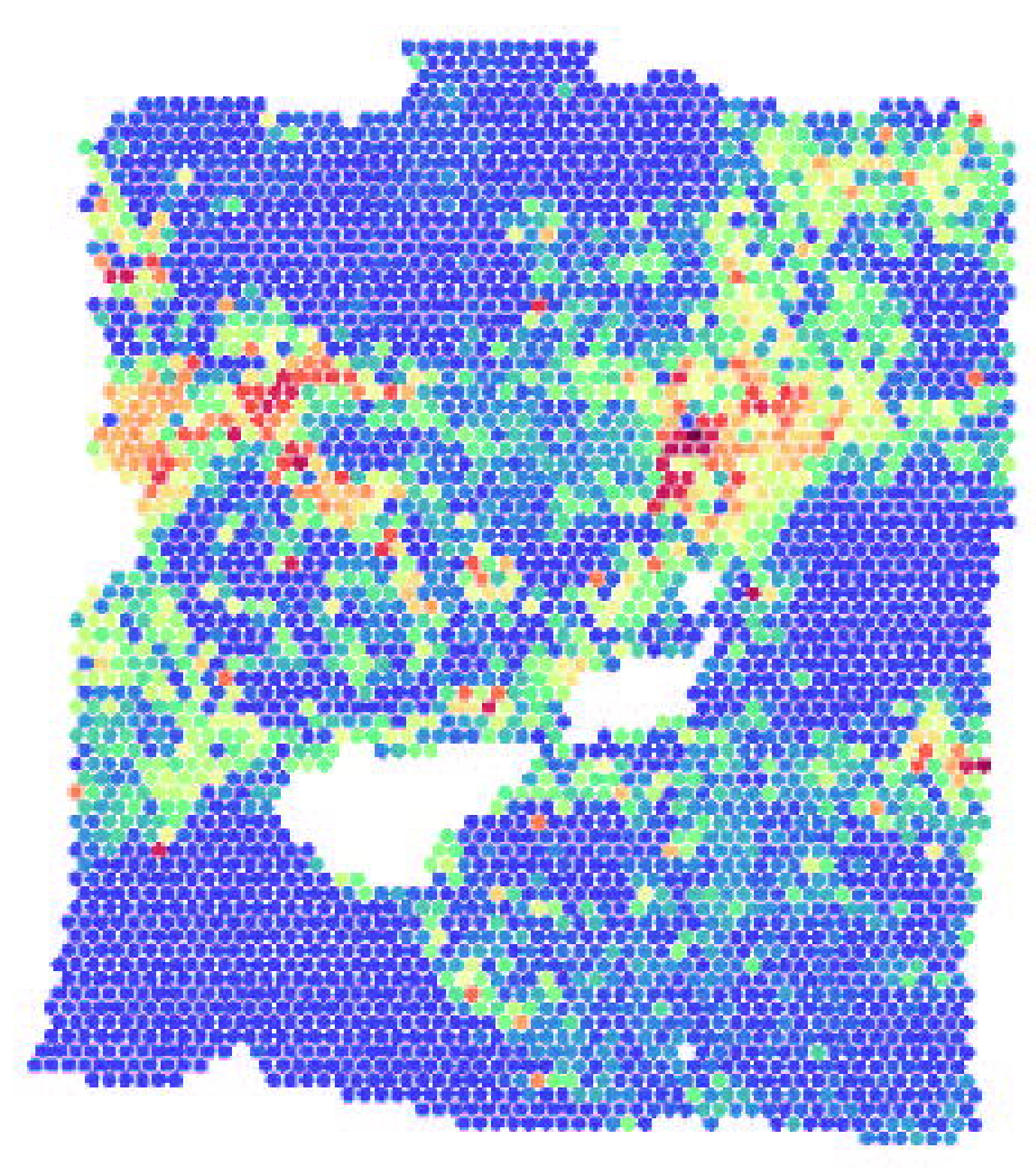

\section{C-EPI}

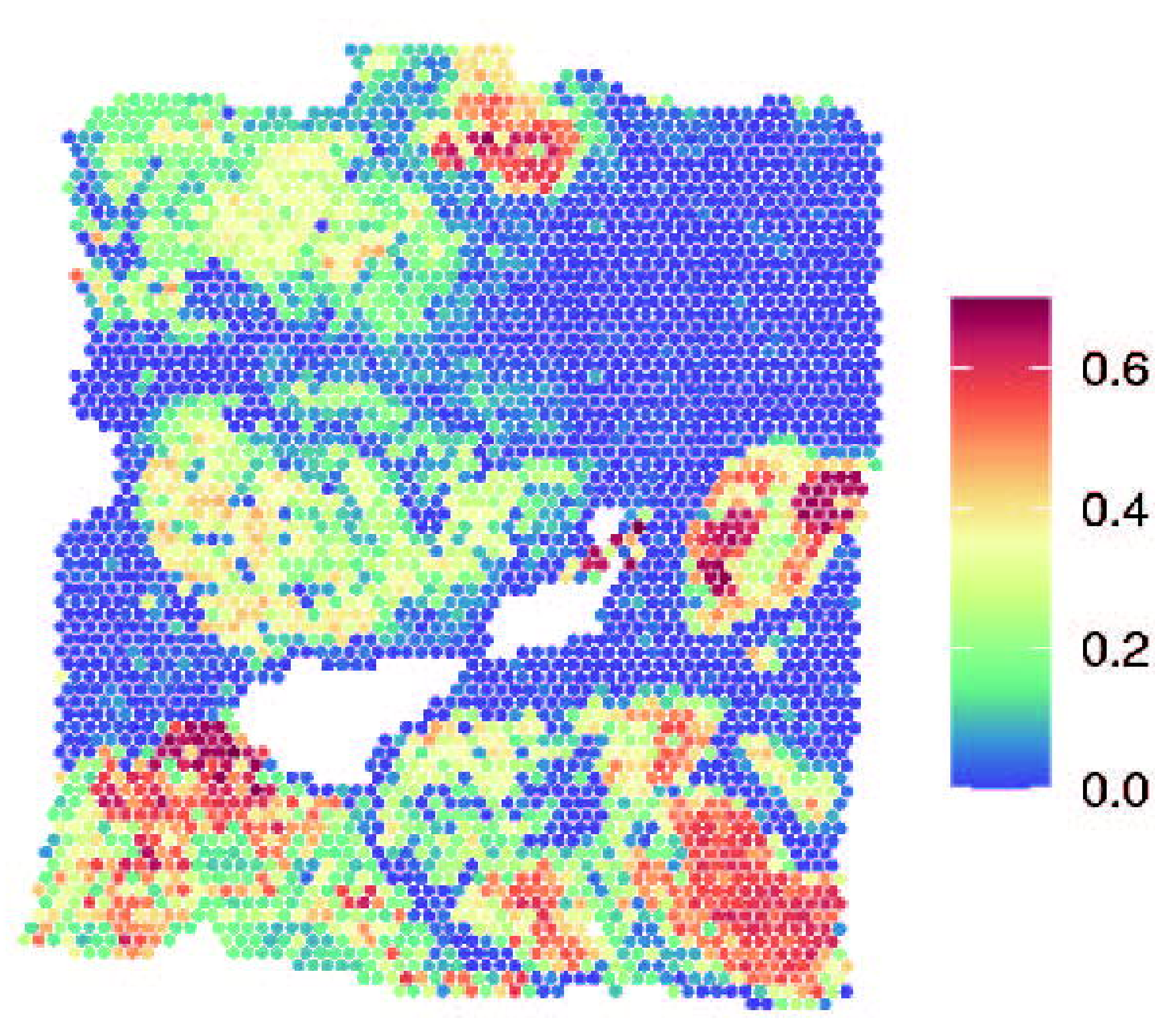

E

\section{PAGA}

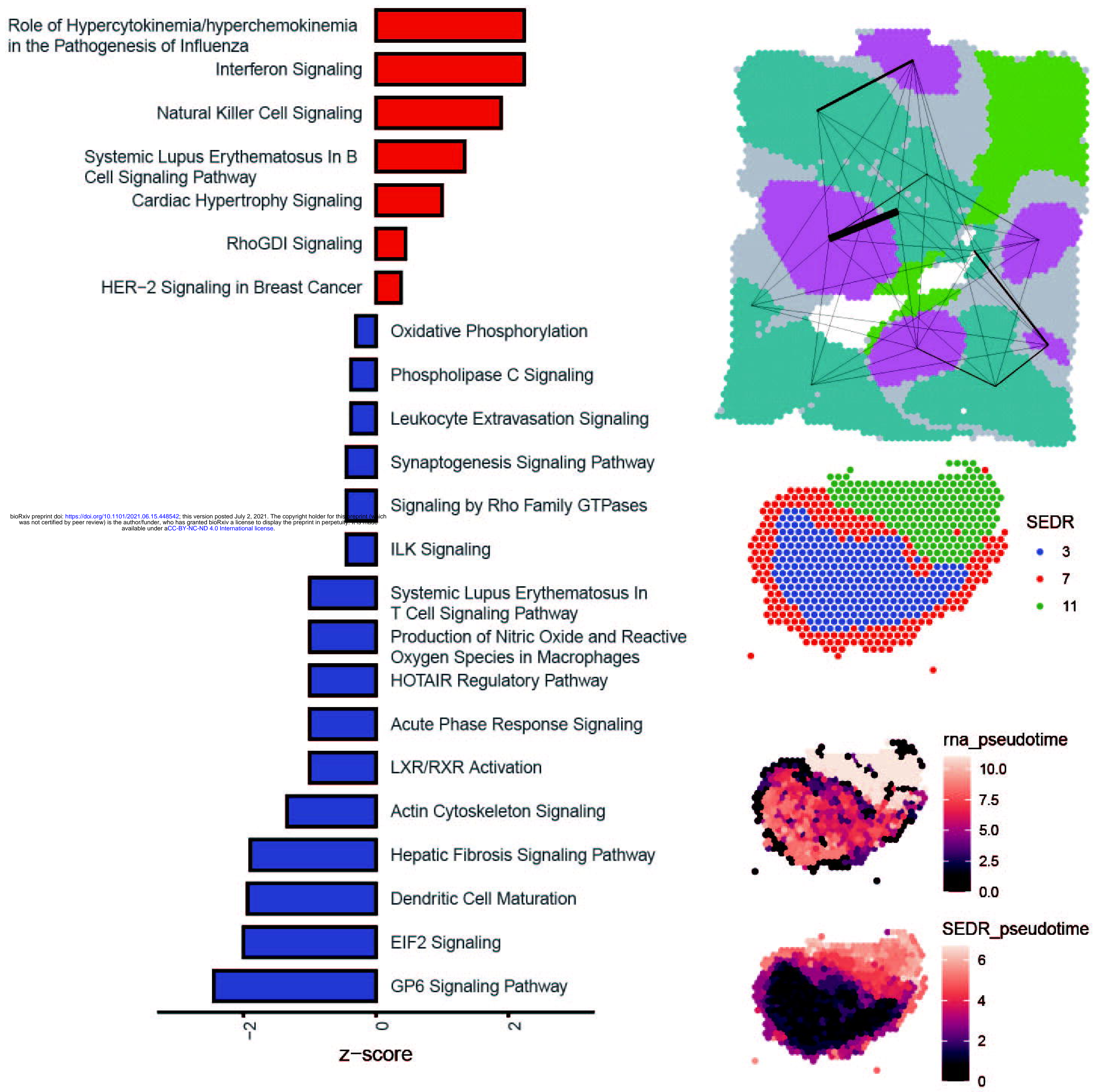

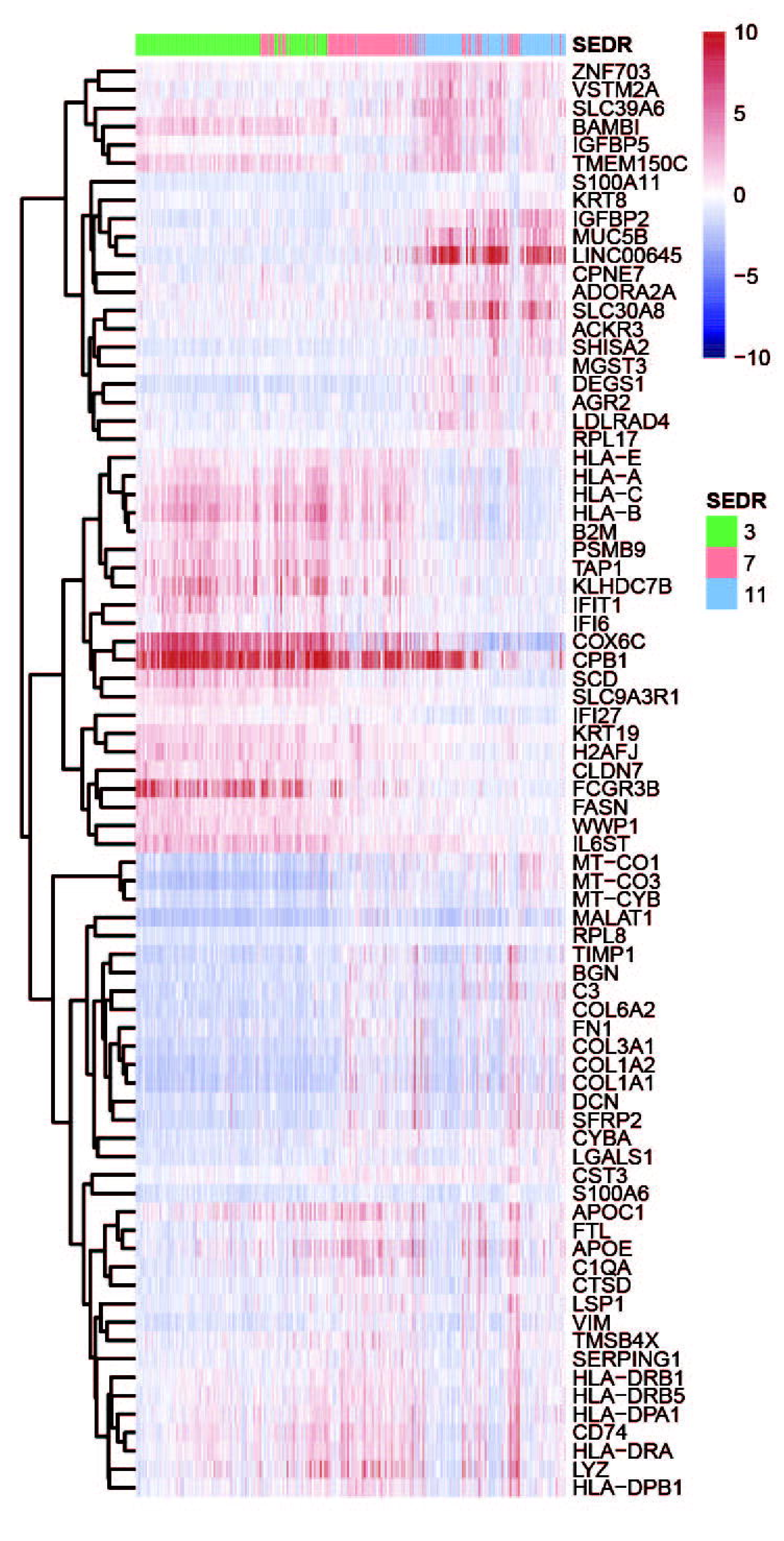




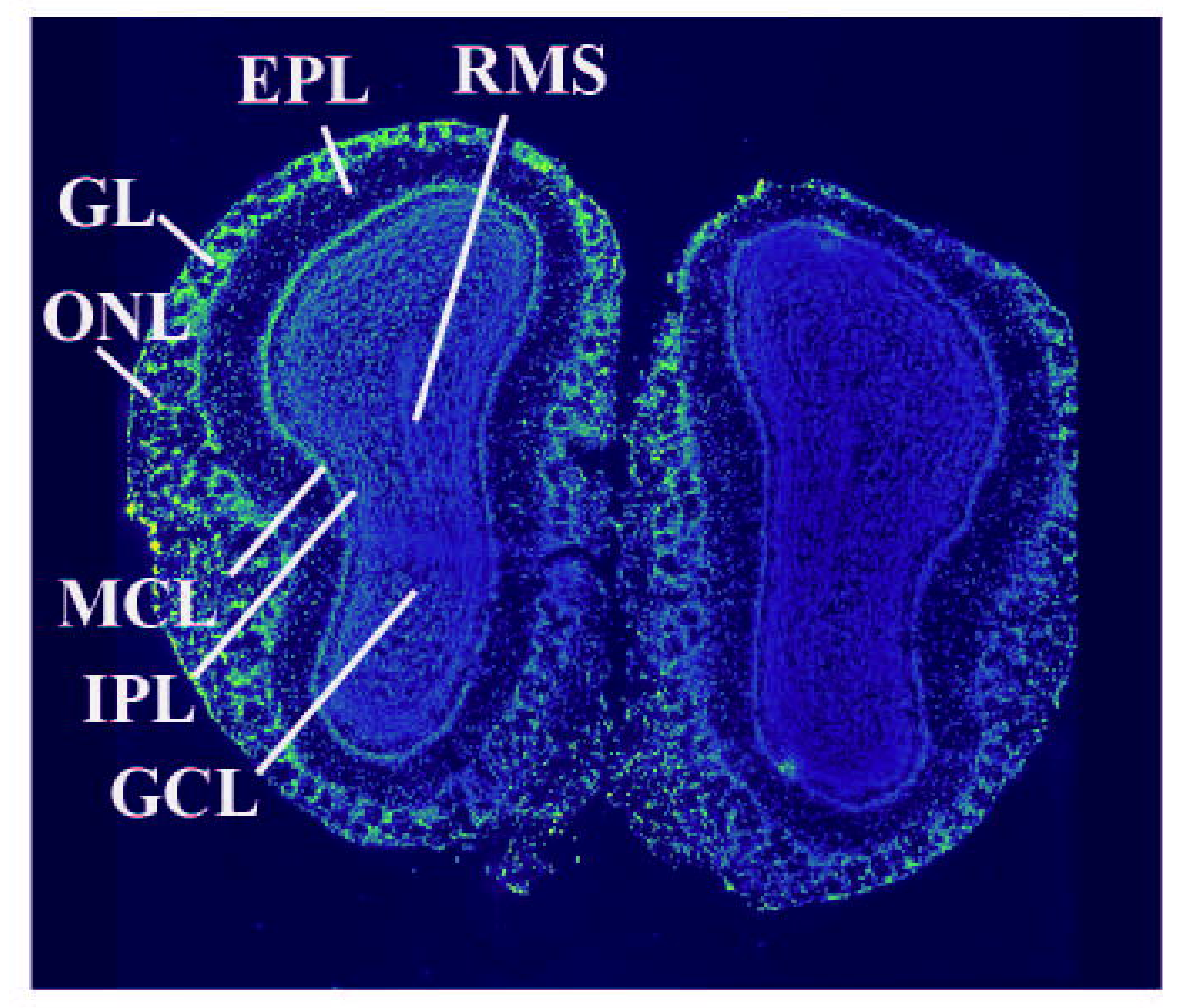

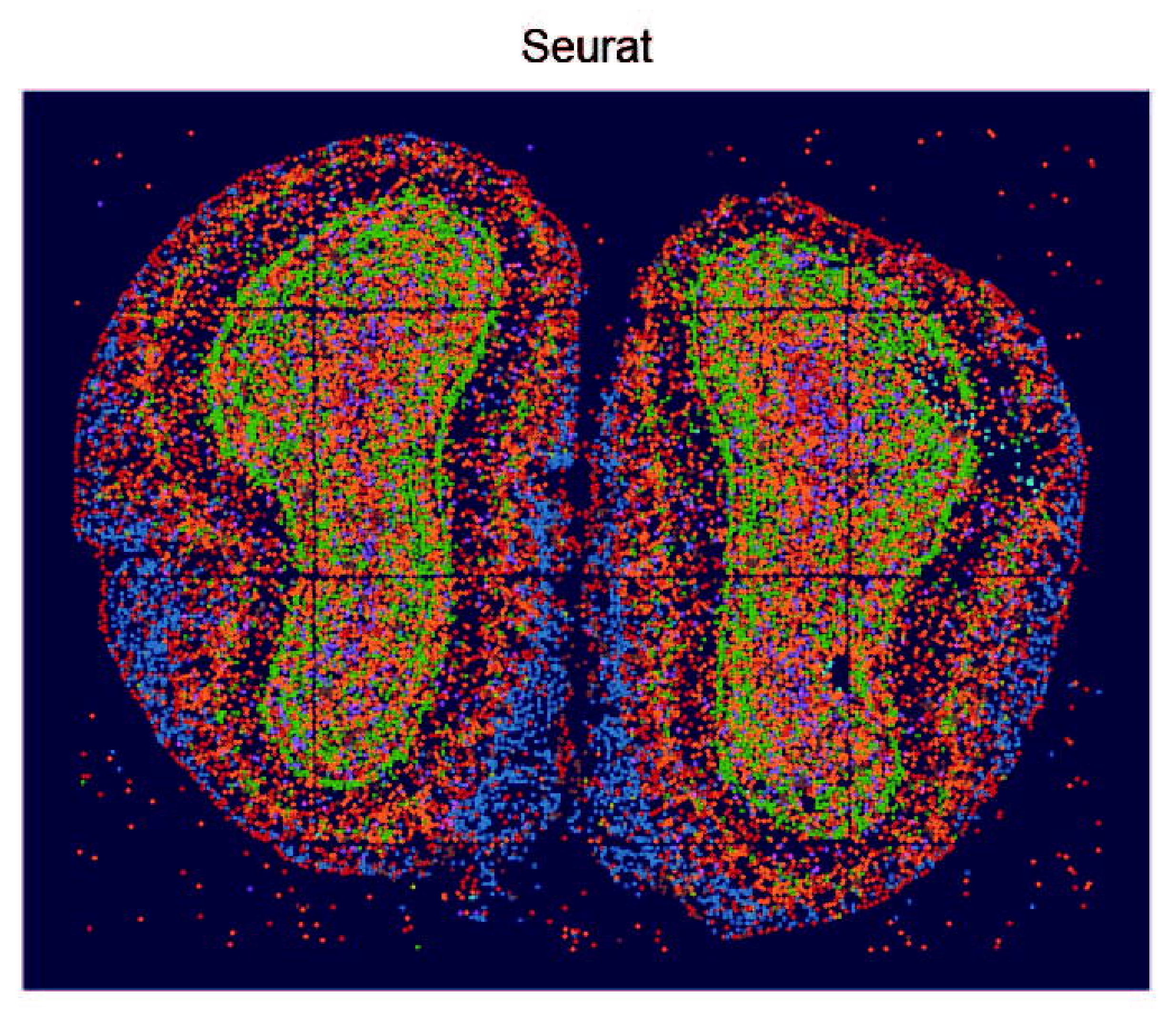

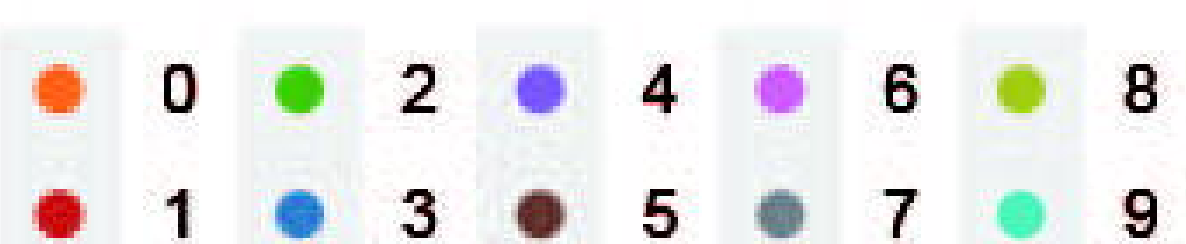

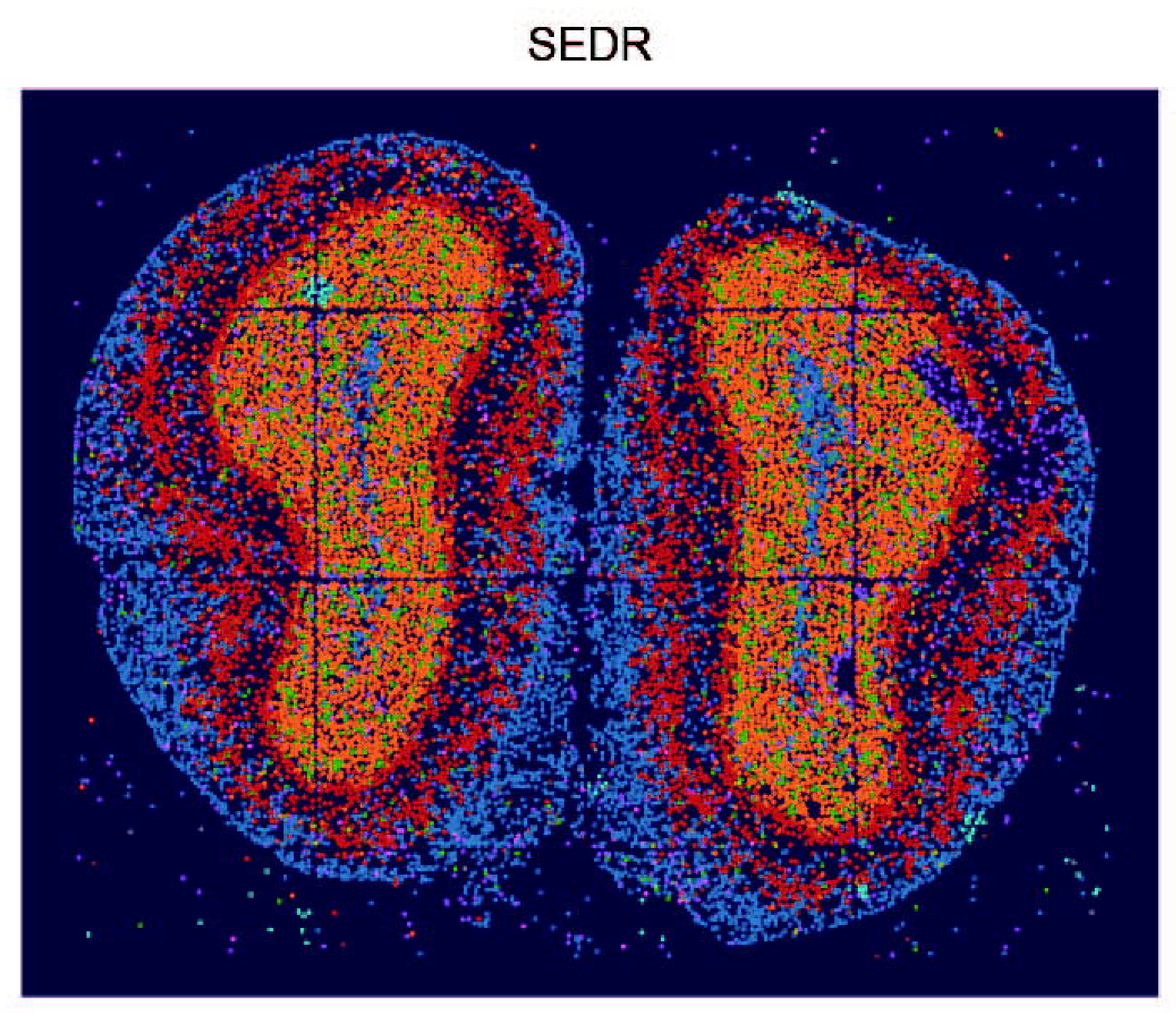

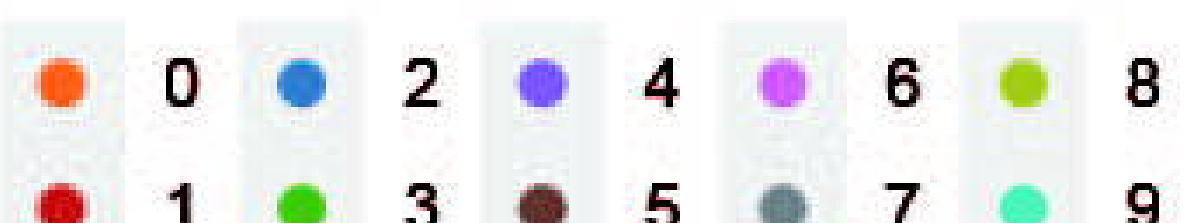

$\mathrm{C}=$

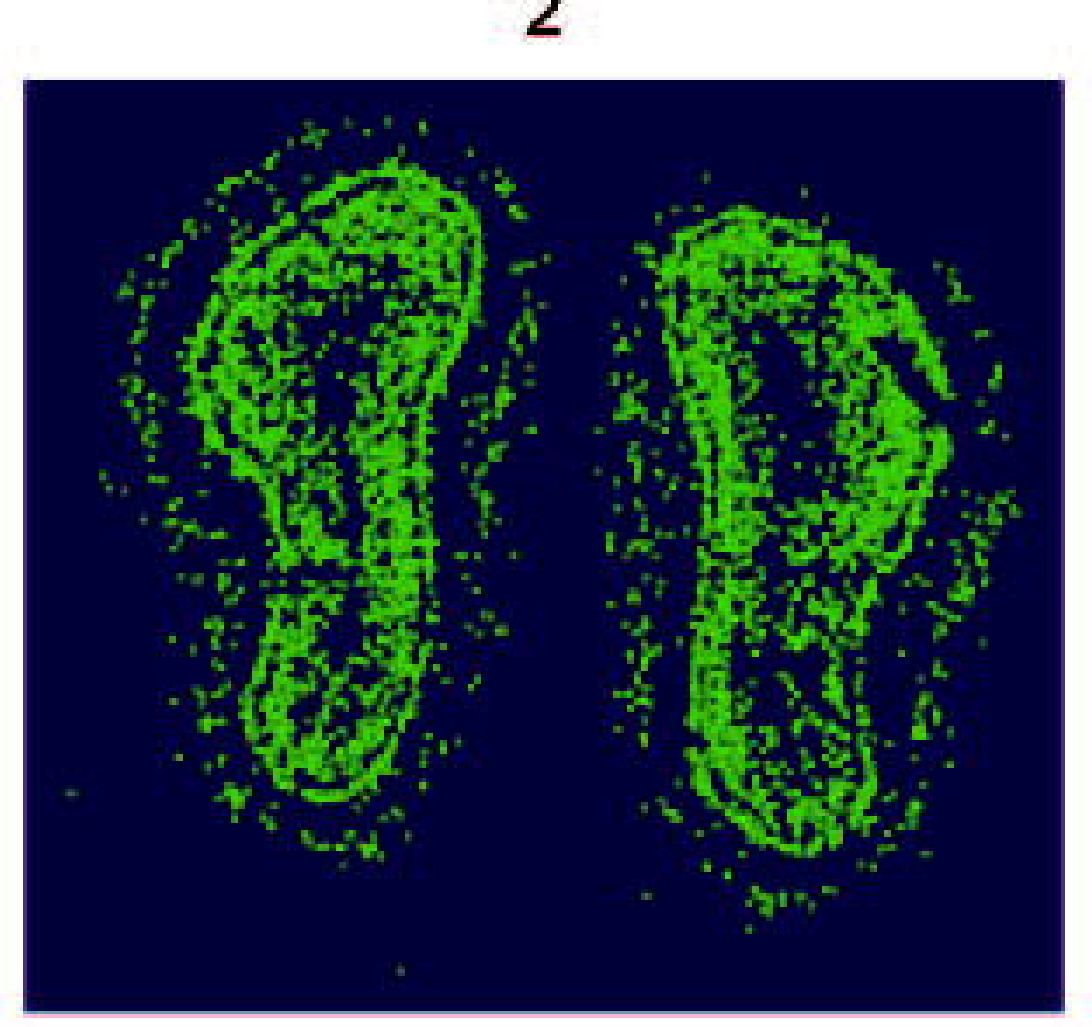

3

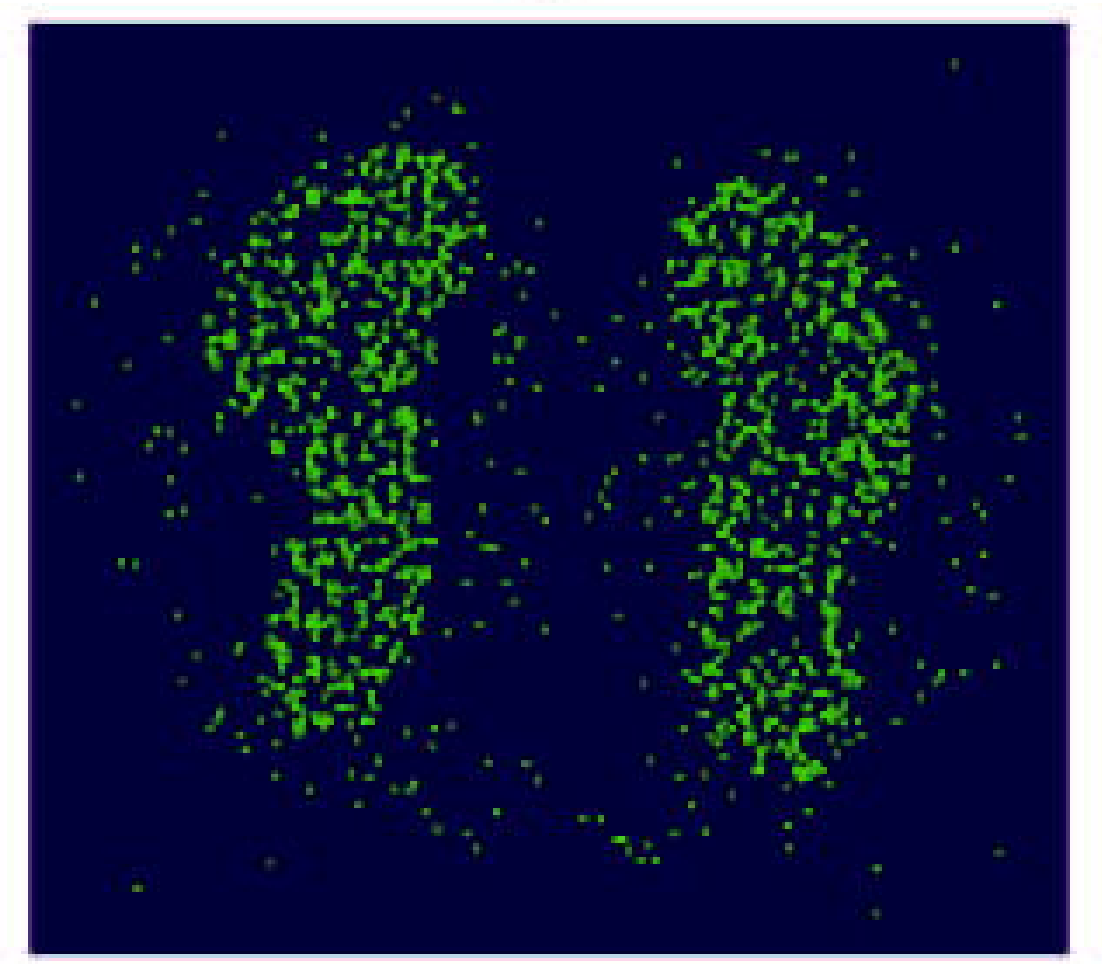

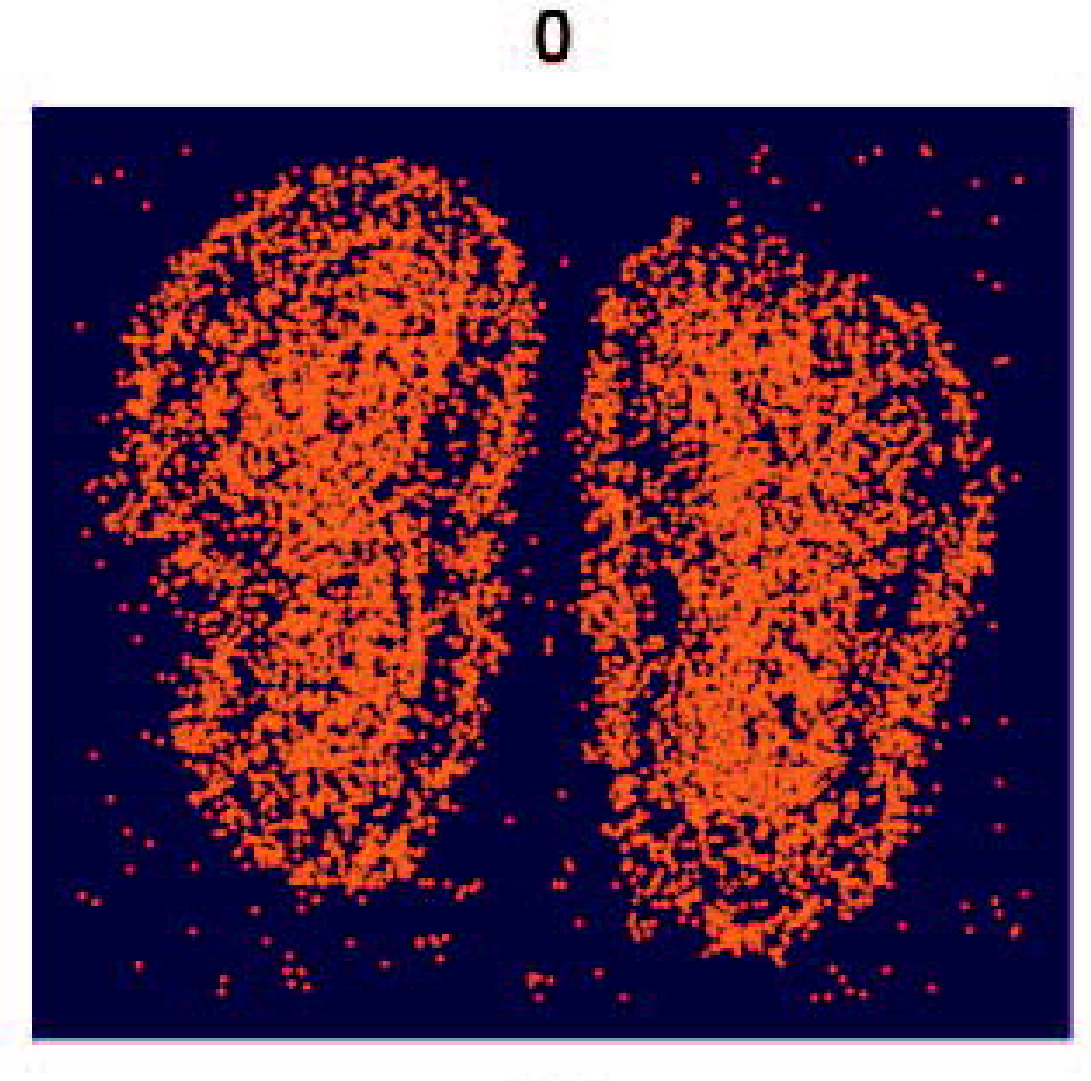

0

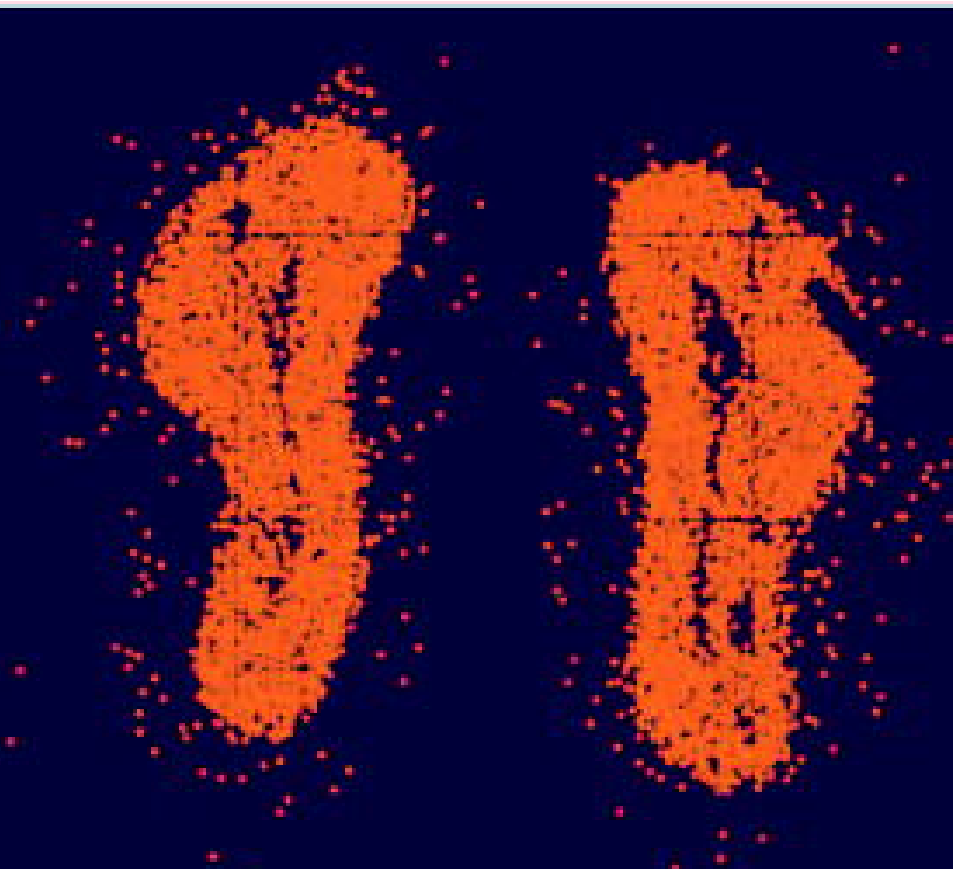

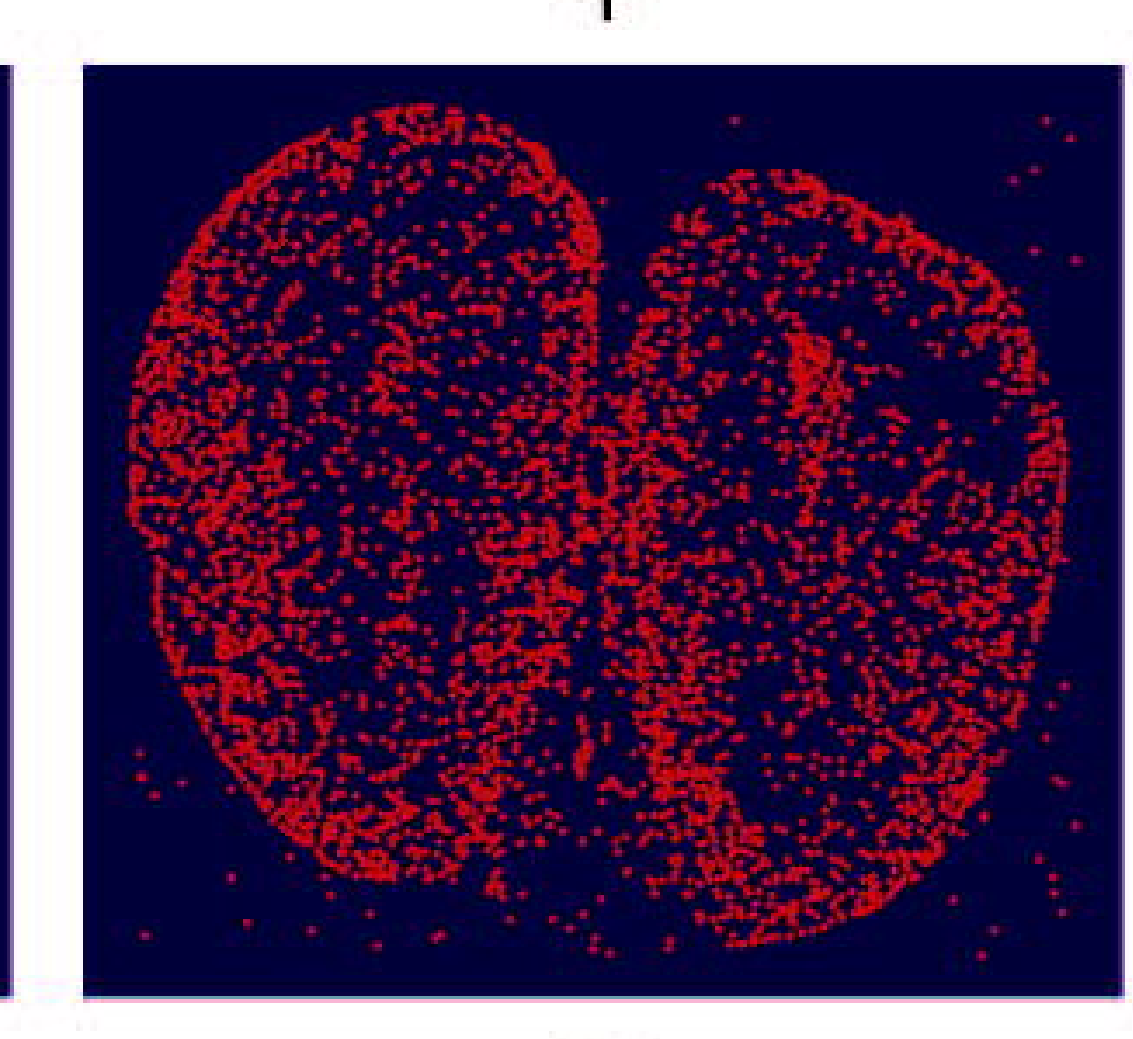

1

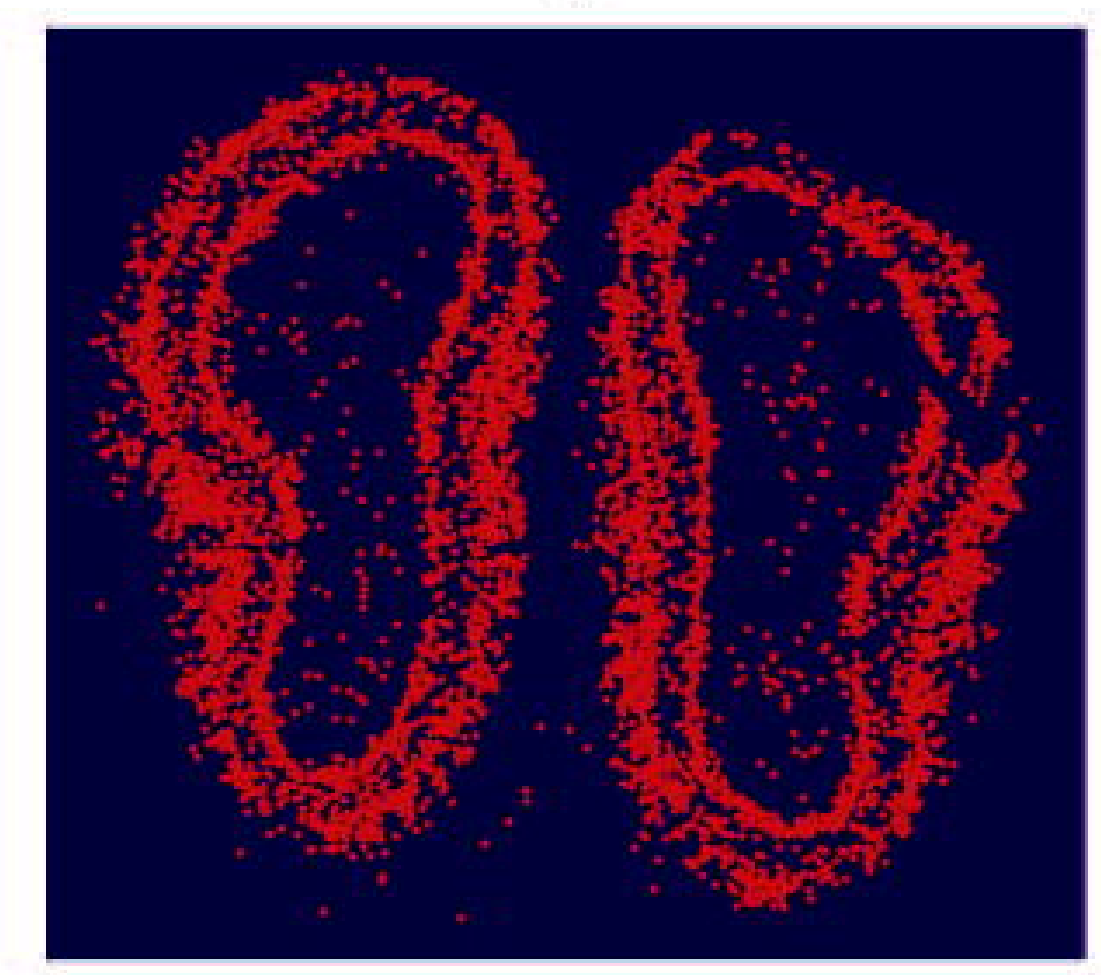

D

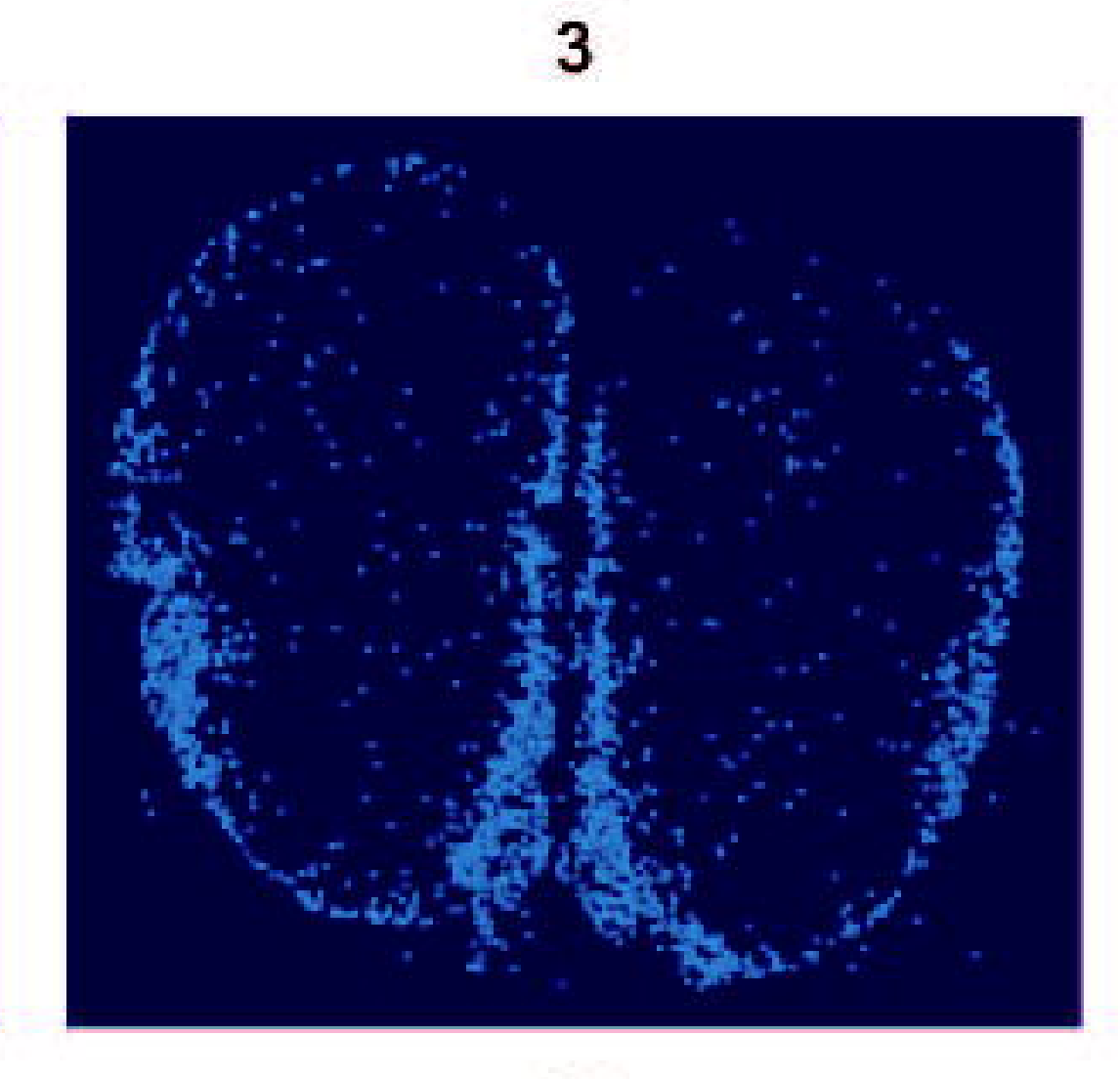

2

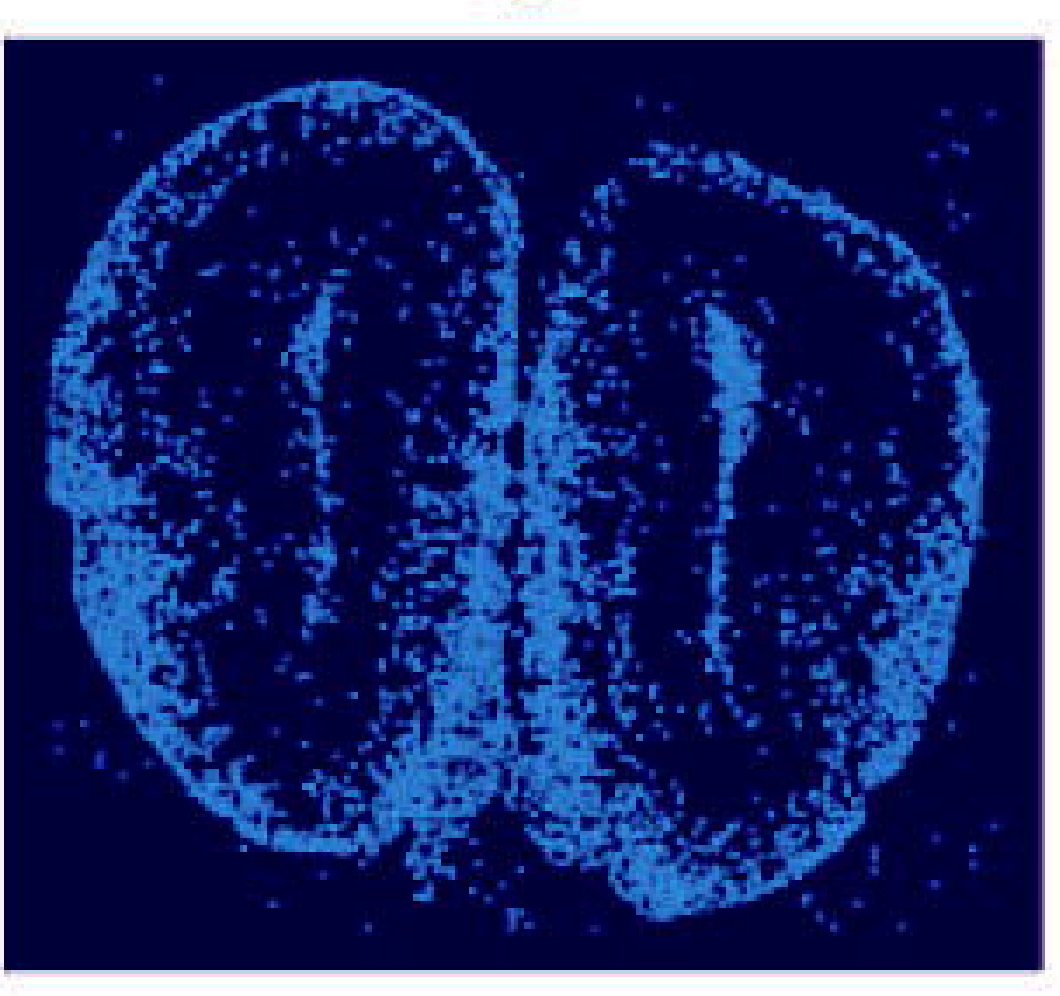

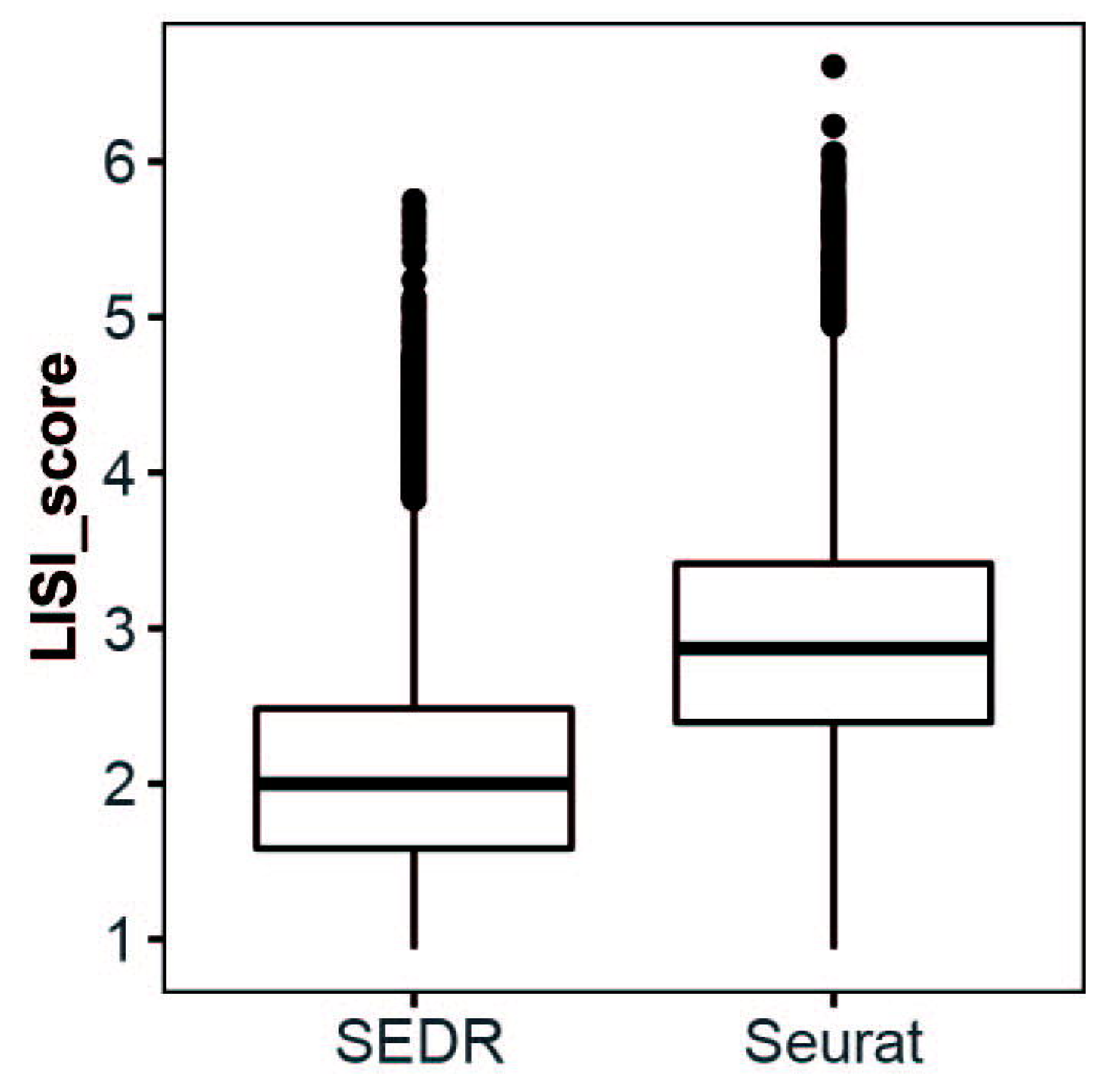




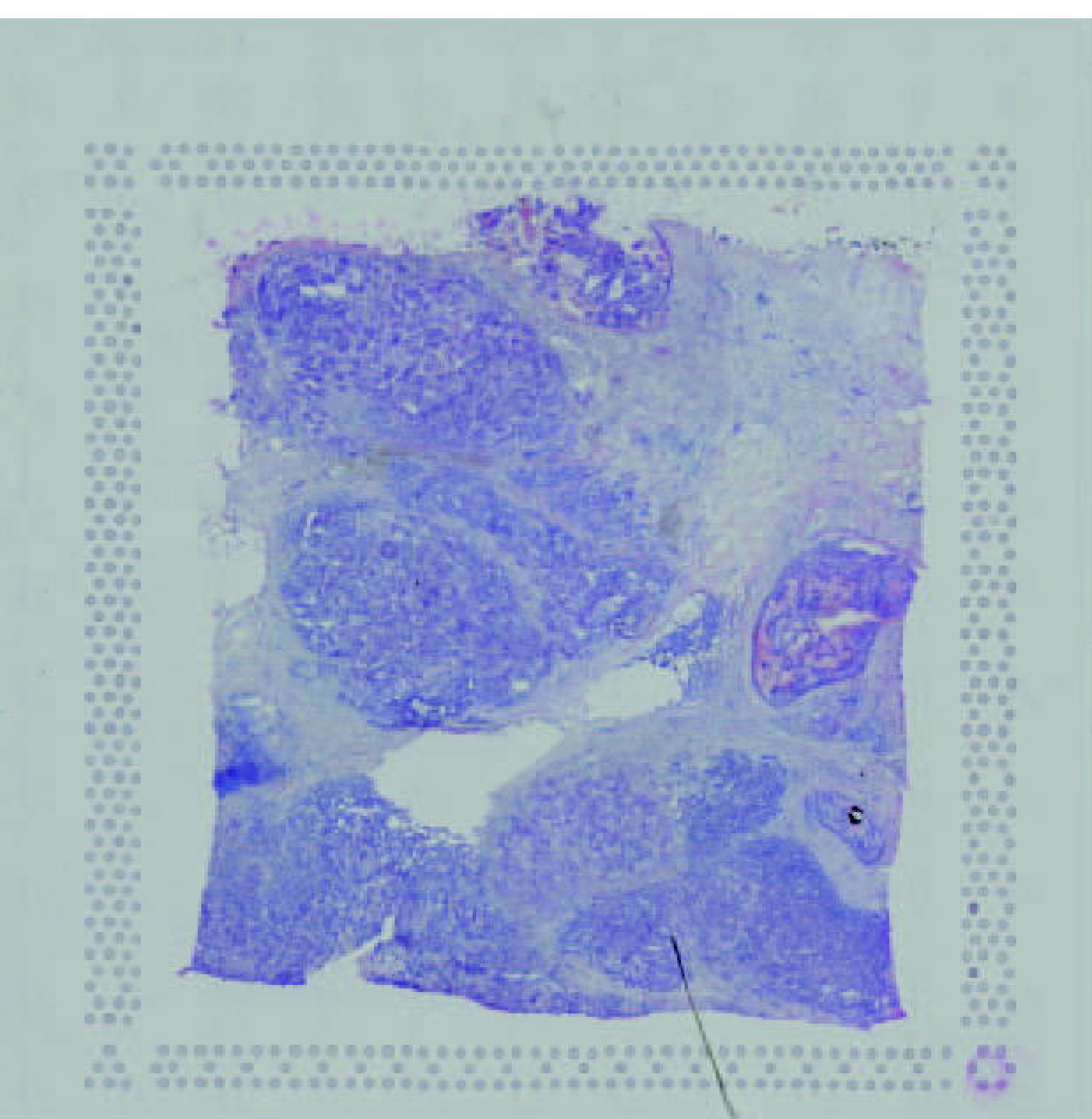

B

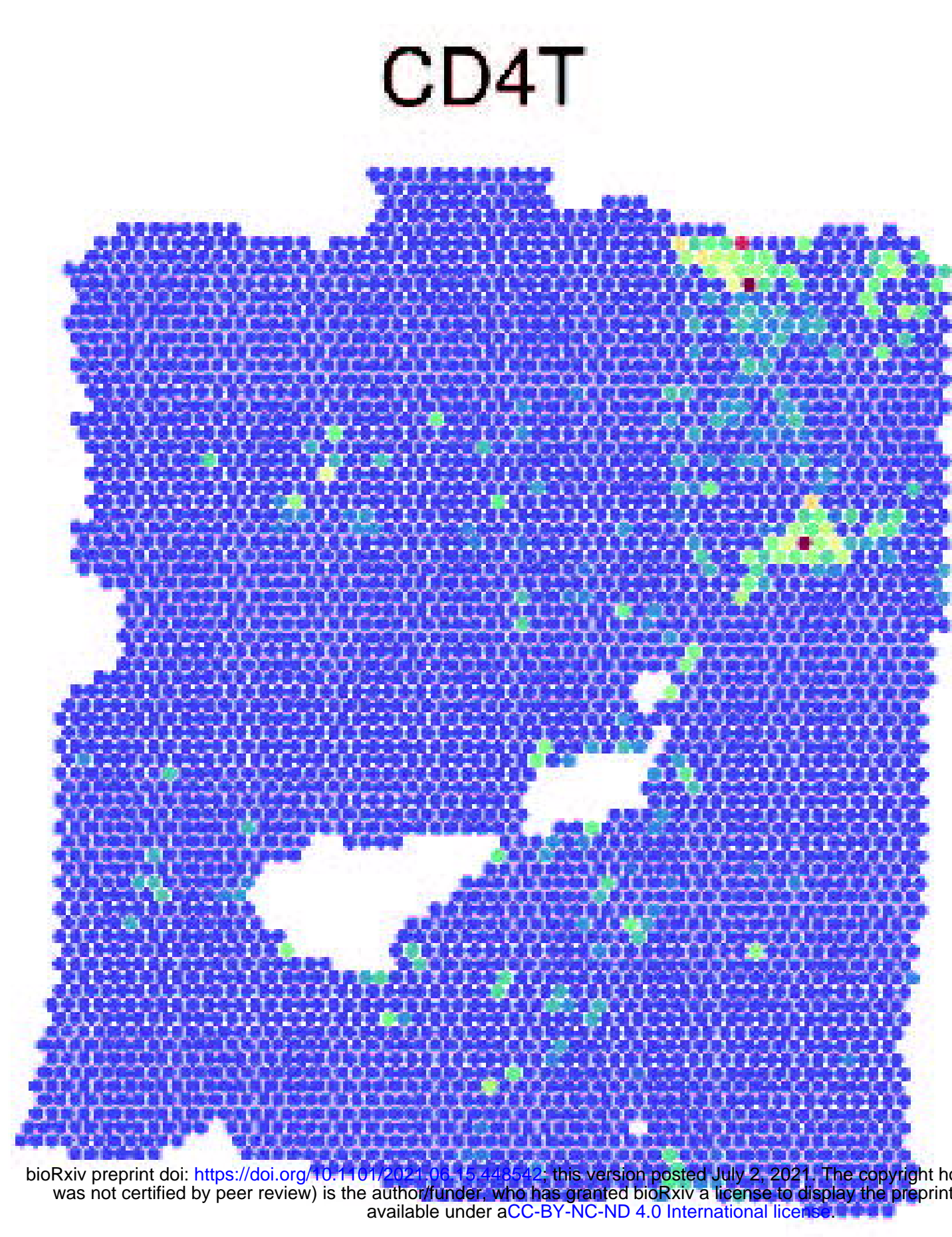

0.000 .020 .040 .060 .08

Tumor-associated-macrophages

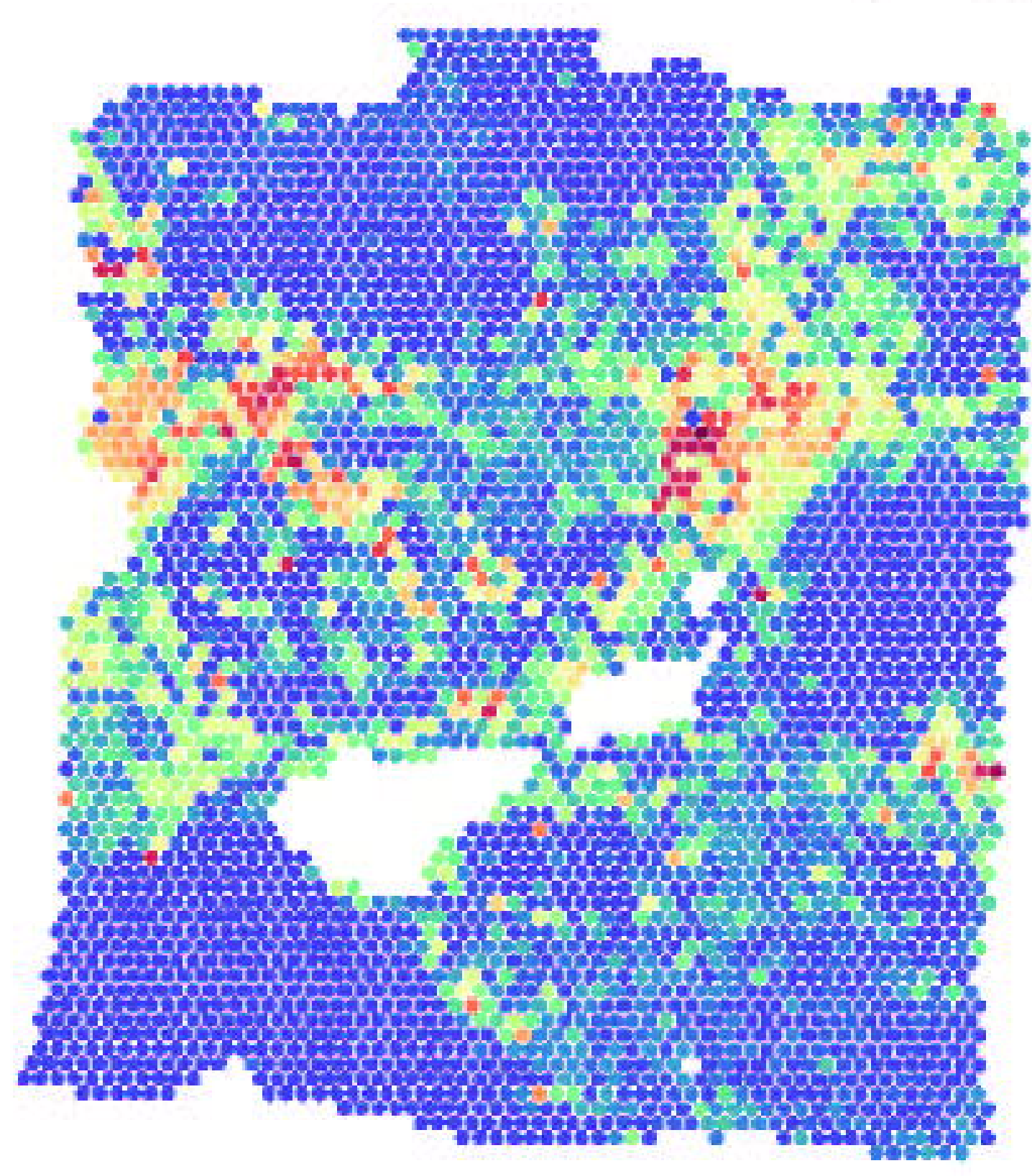

$\begin{array}{lllll}0.0 & 0.2 & 0.4 & 0.6 & 0.8\end{array}$ macrophages

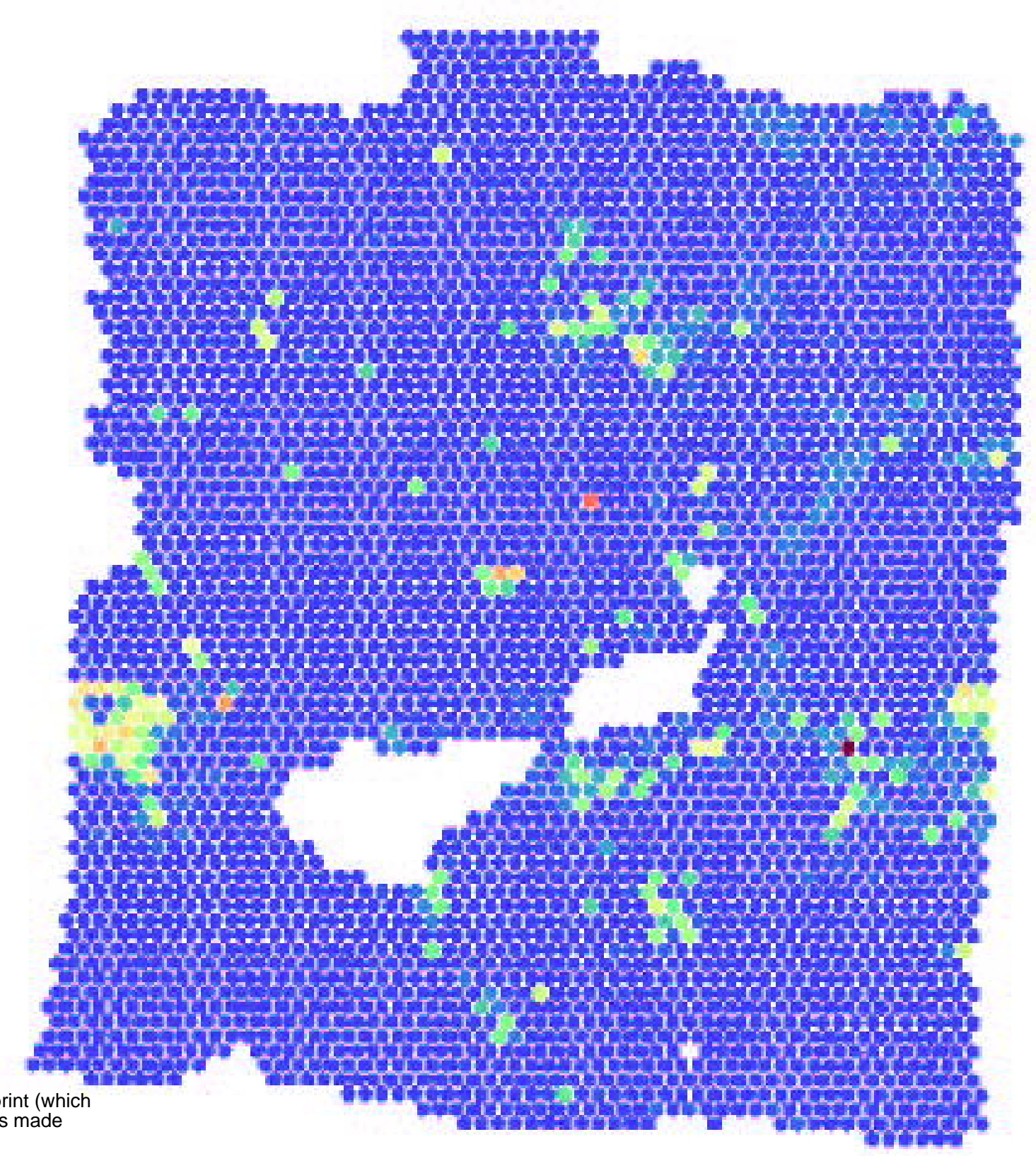

$\begin{array}{llll}0.0 & 0.1 & 0.2 & 0.3\end{array}$

Epi-Basal

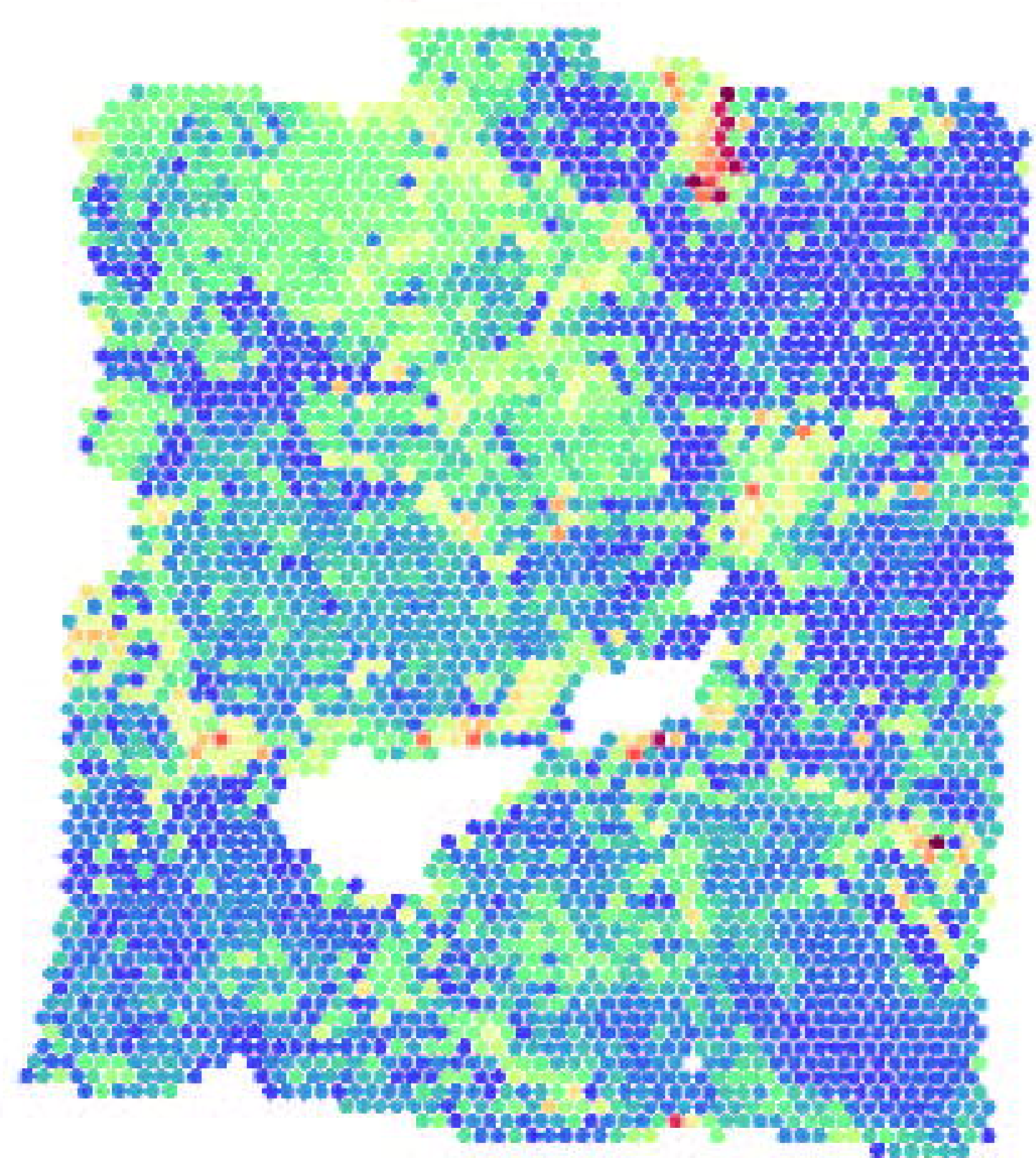

Fibroblast

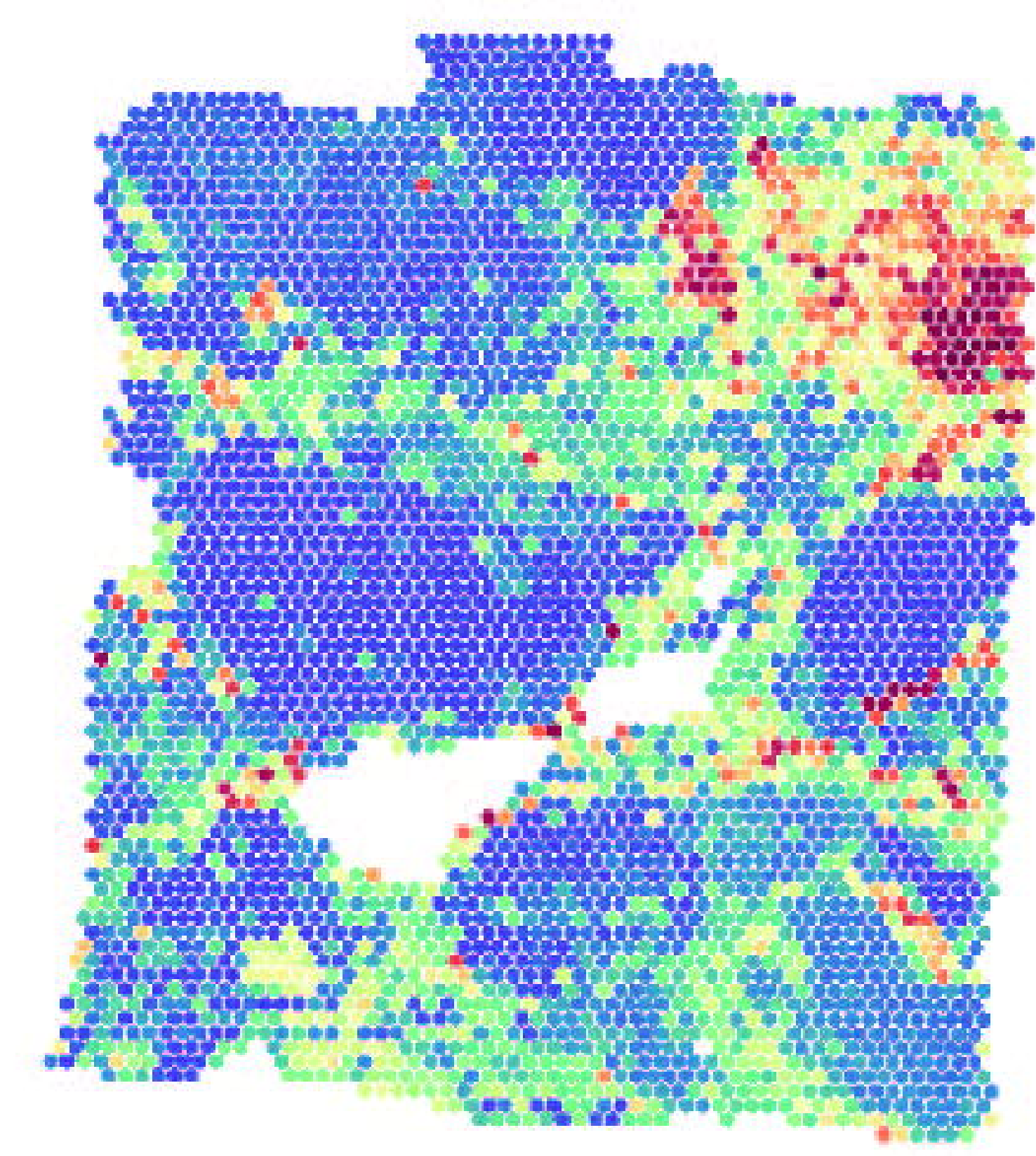

12.

$\begin{array}{lllll}0.00 & 0.25 & 0.50 & 0.75 & 1.00\end{array}$ Epi

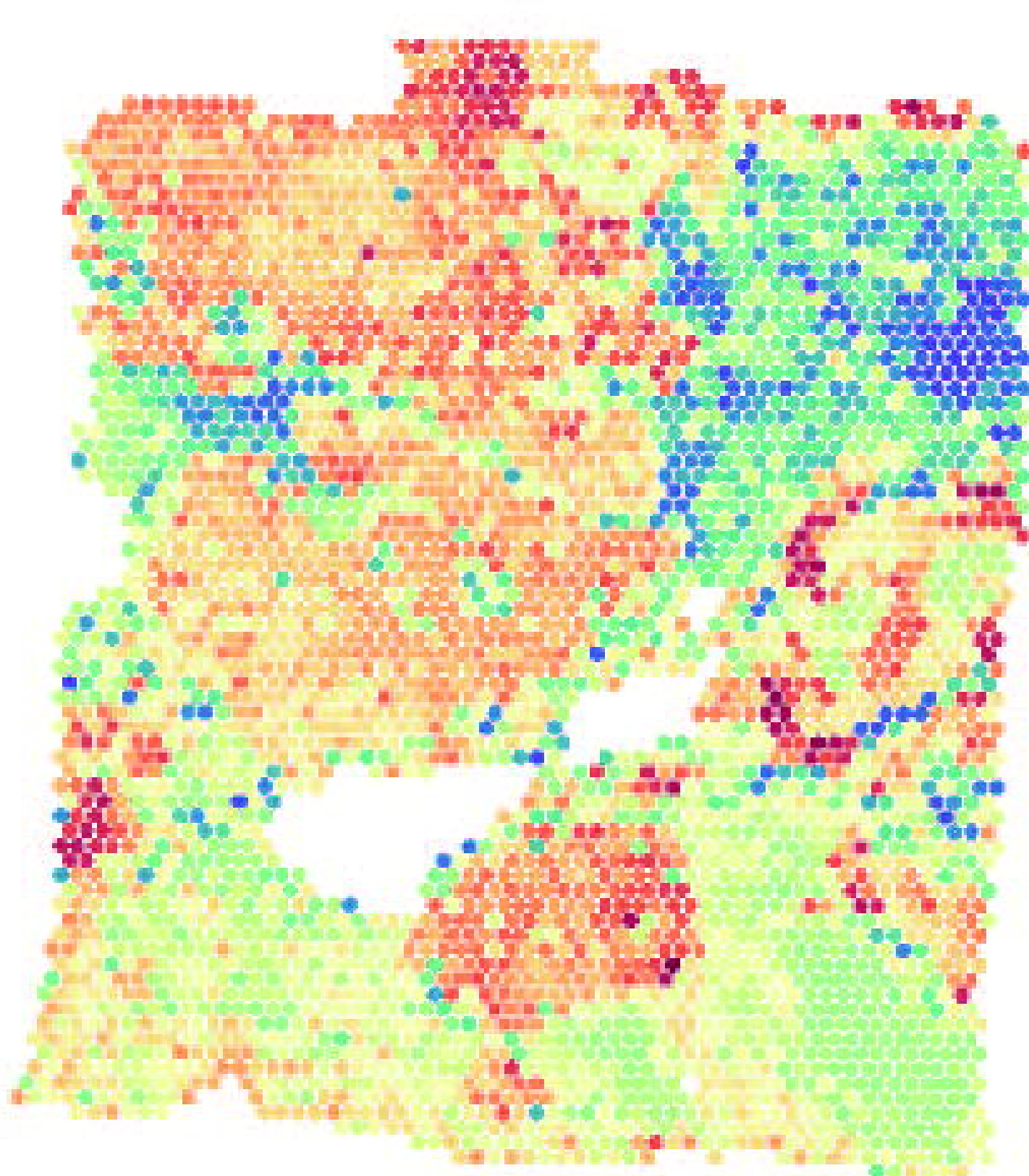

Endo

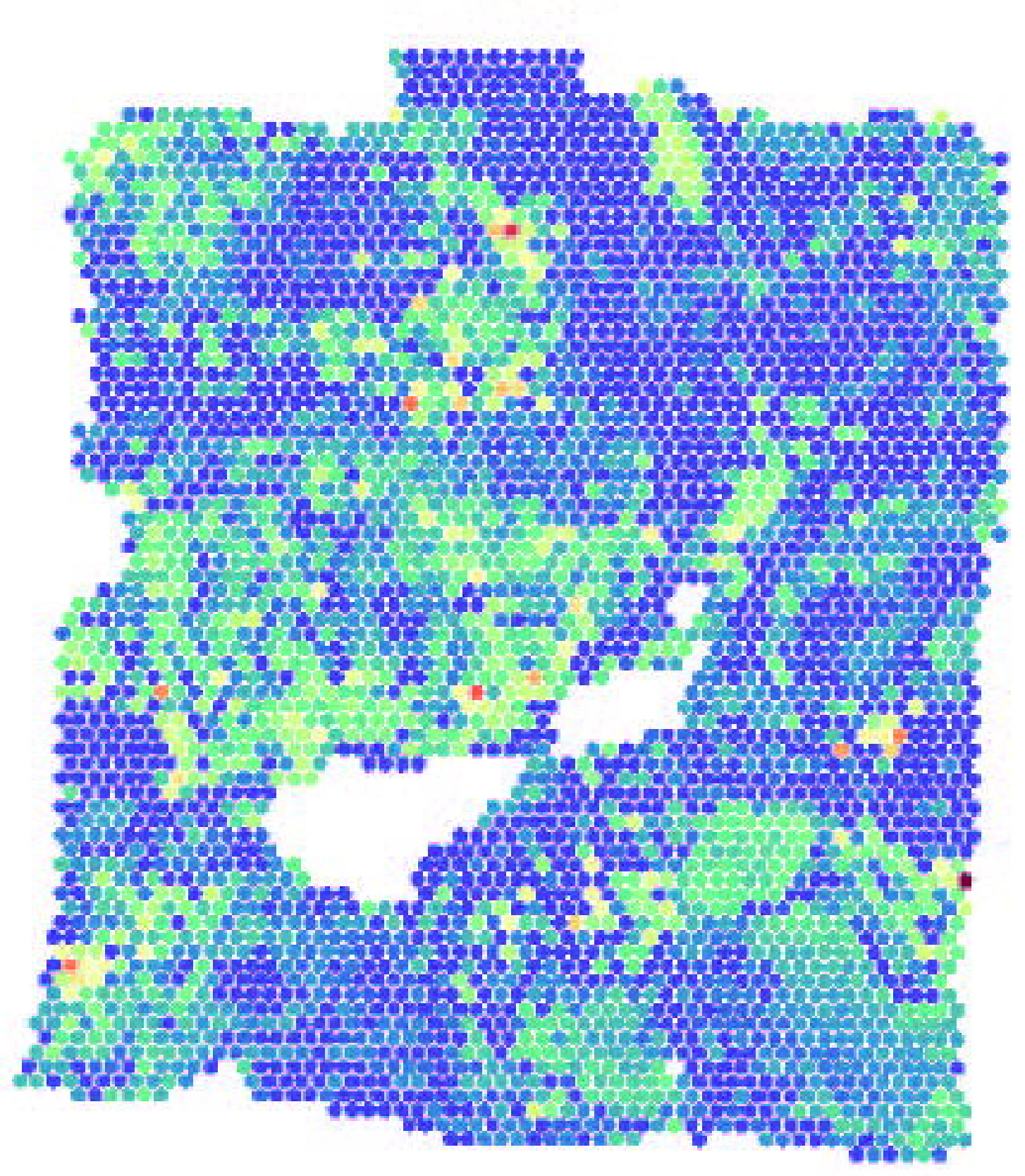

0.000 .050 .100 .150 .20 Cycling-Epi

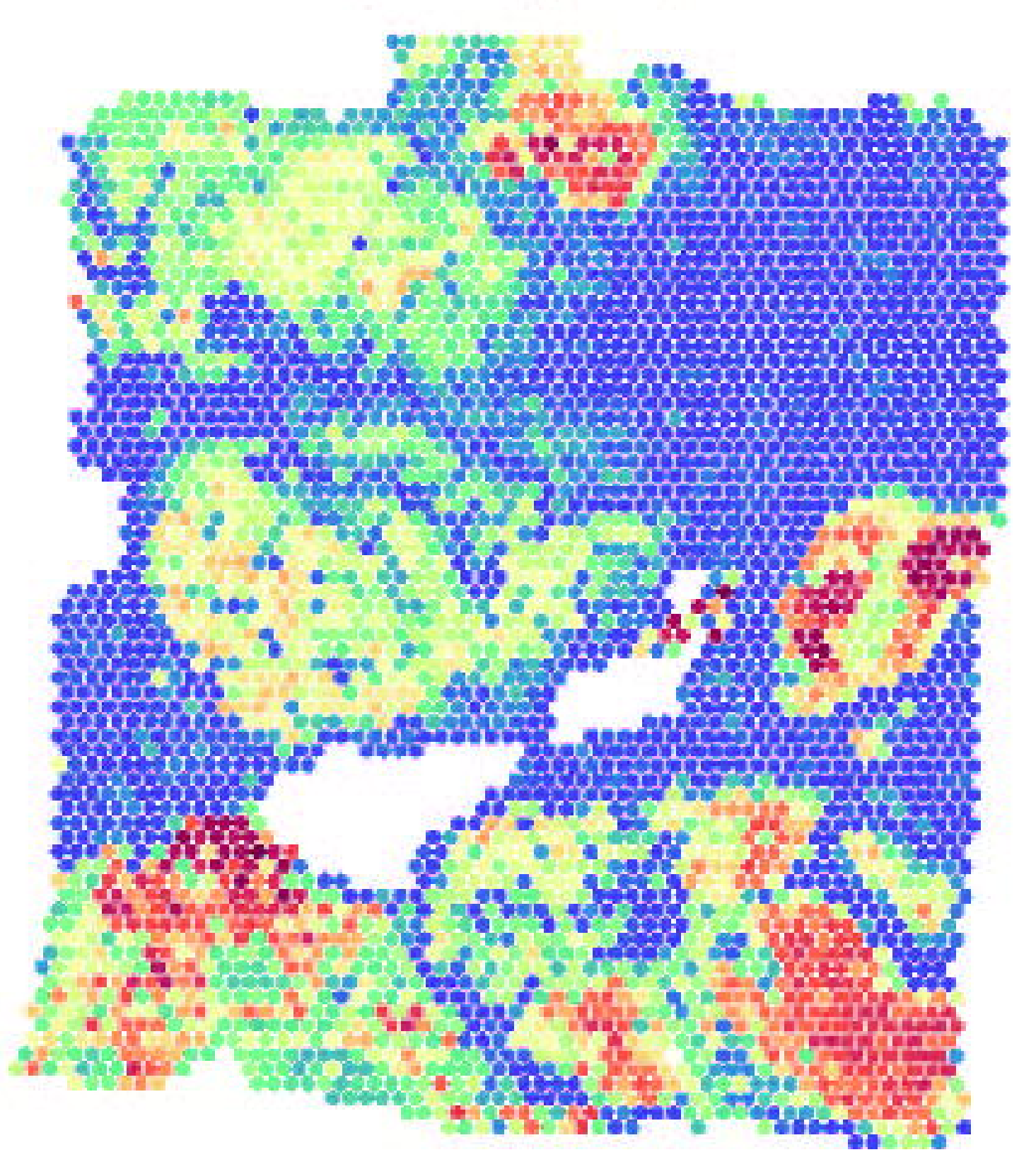

Plasma

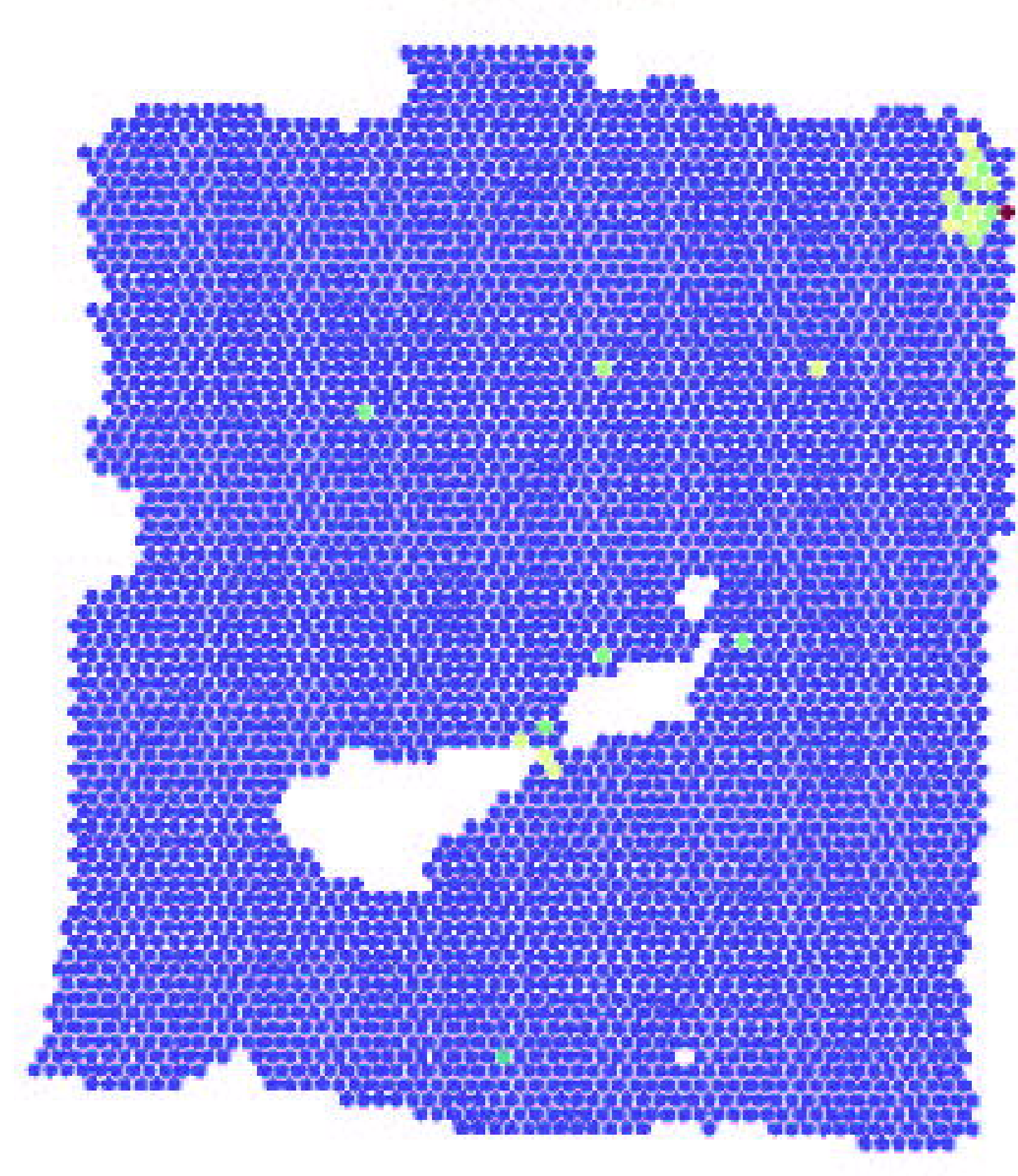

$\begin{array}{lll}0.00 & 0.01 & 0.02\end{array}$ 

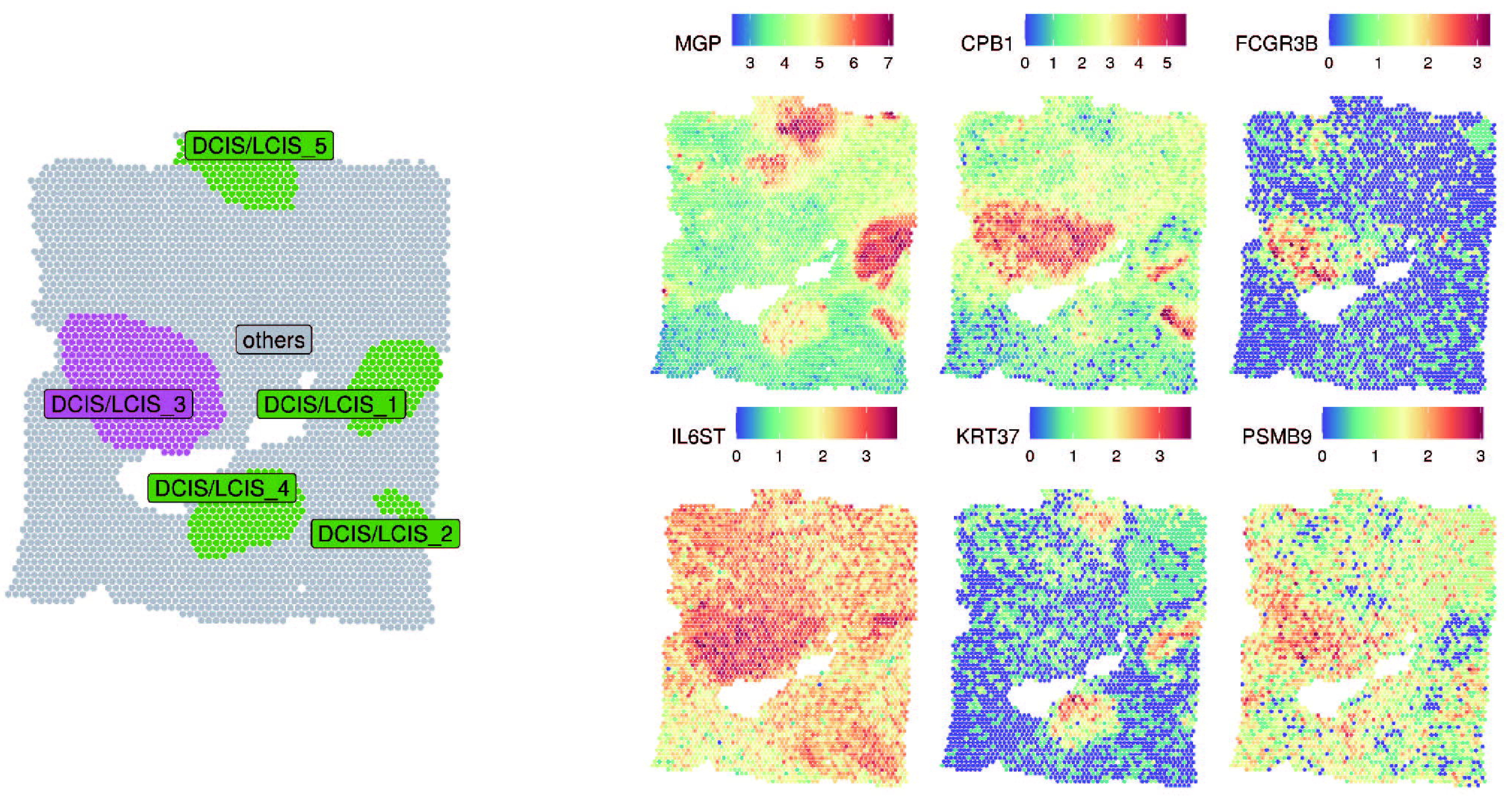

$\mathbf{E}$

Dendritic Cell Maturation

T Cell Receptor Signaling PKC.. Signaling in T Lymphocytes Role of NFAT in Regulation of the Immune Response Th1 Pathway

Crosstalk between Dendritic Cells and Natural Killer Cells iCOS-iCOSL Signaling in T Helper Cells rythematosus In T Cell Signaling Pathway Fc.. Receptor-mediated Phagocytosis in Macrophages and Monocytes

Regulation Of The Transition By Grown Faclias Pathchymal T Cel Exhaution Factors Pathway

Cell Exhaustion Signaling Pathway

HER-2 Signaling in Breast Cancer Interferon Signaling Natural Killer Cell Signaling Inflammasome pathway Th2 Pathway Complement System Estrogen Receptor Signaling

D
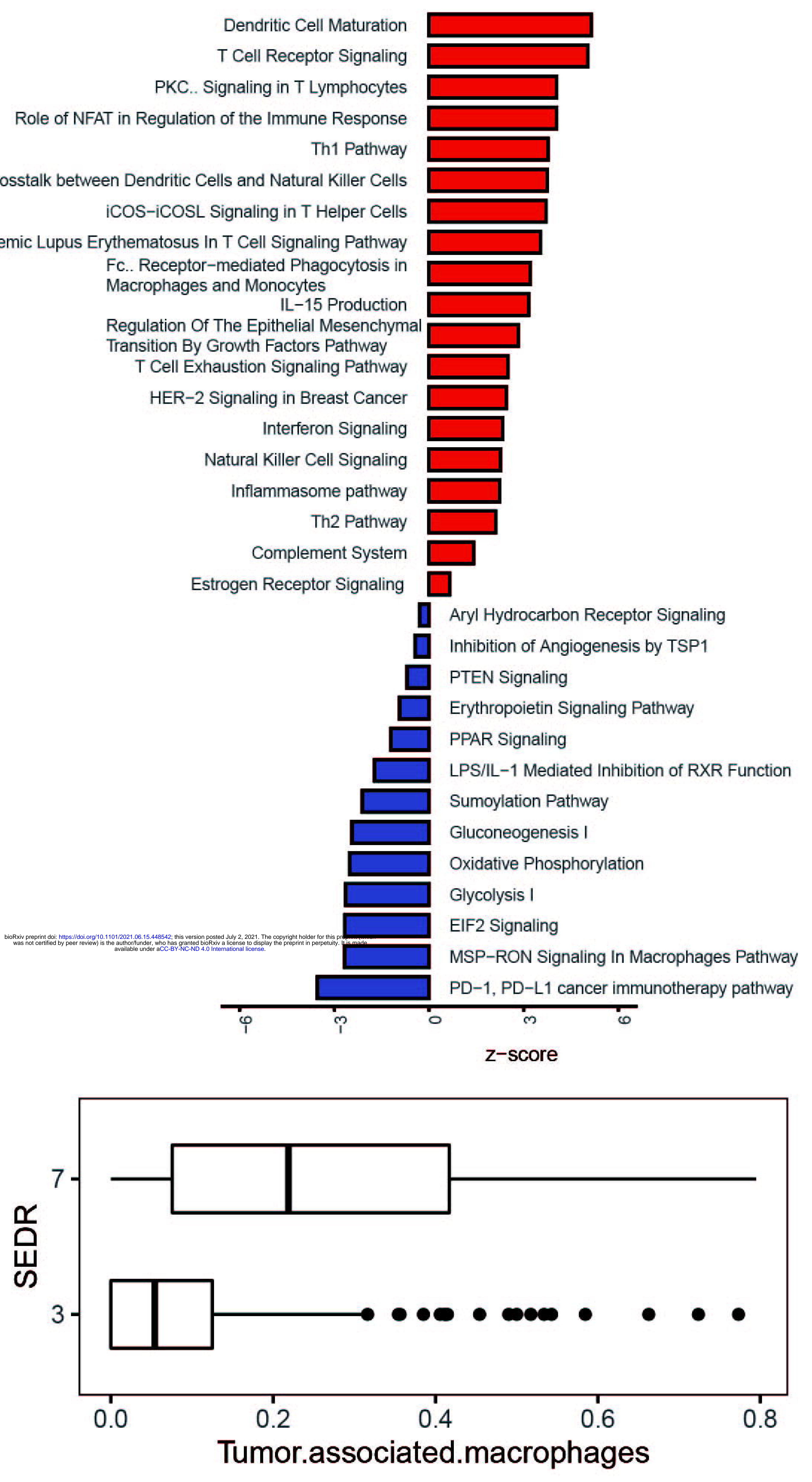
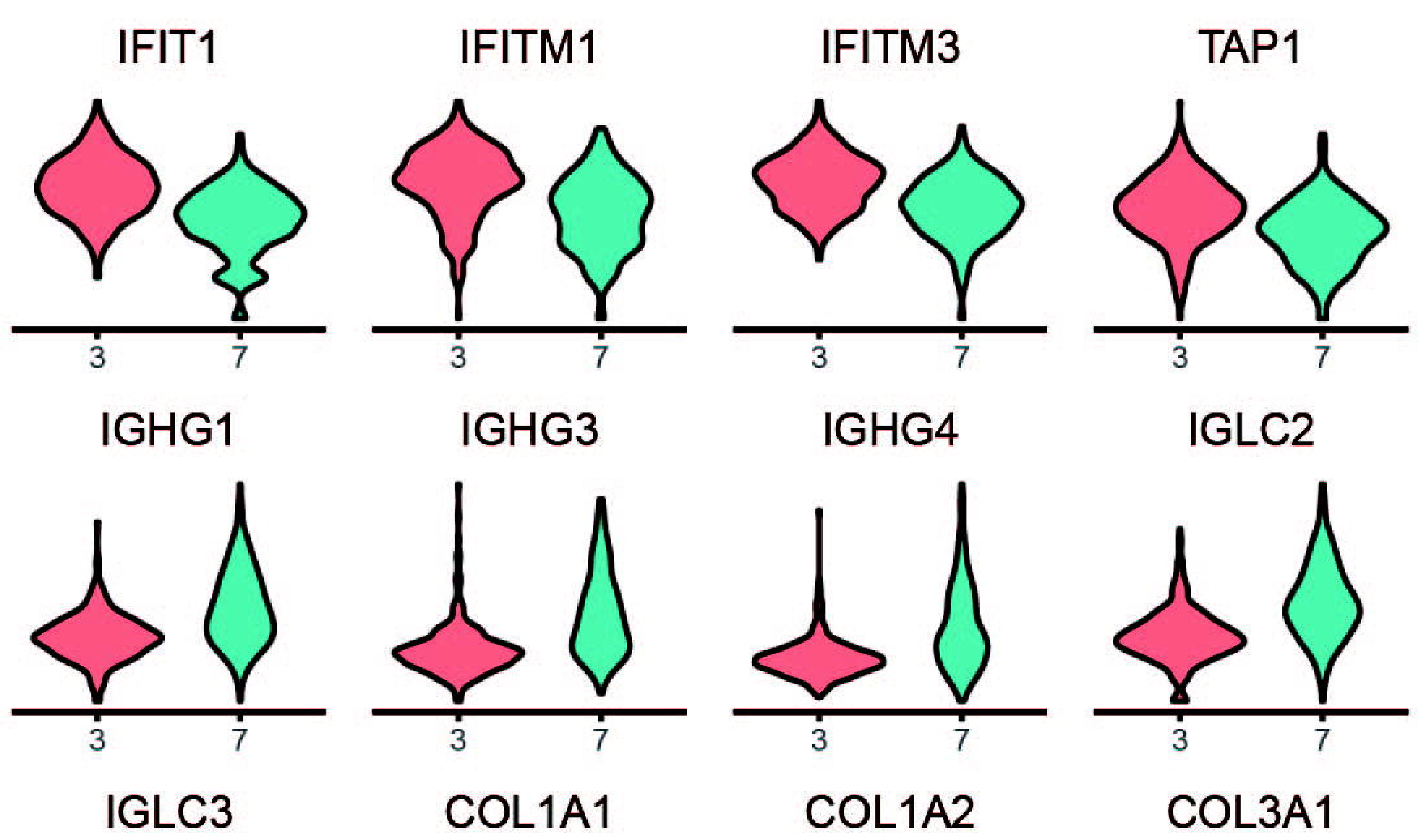

IGLC3

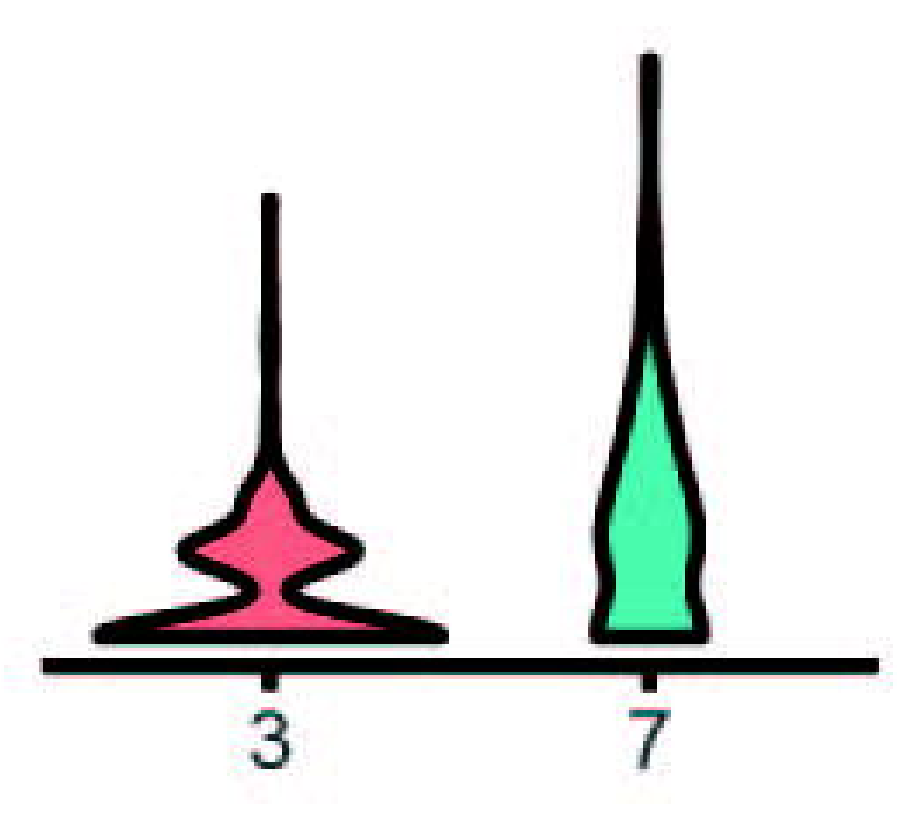

Colitis

COL1A2
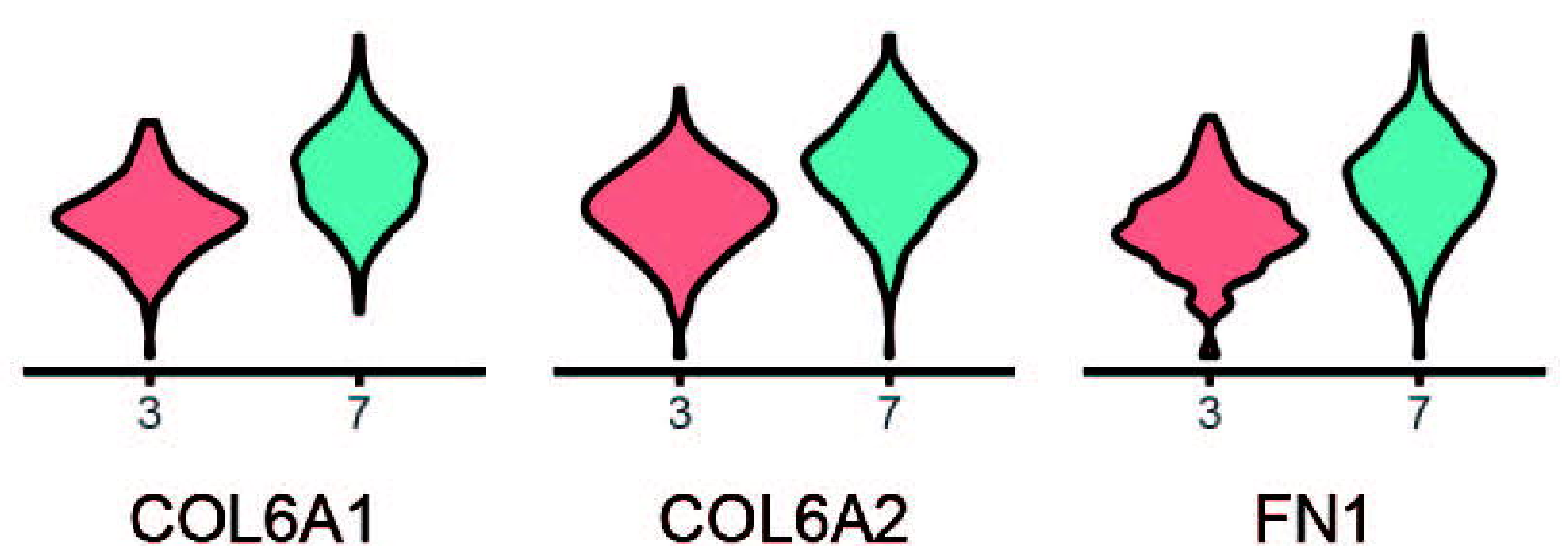

COL5A1
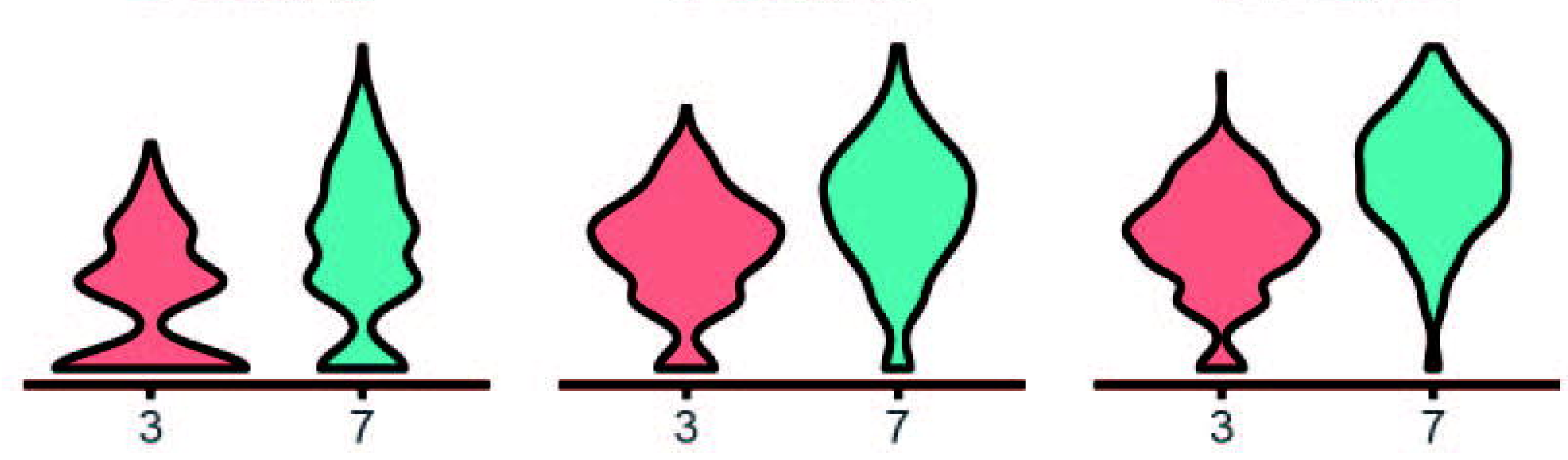

CTSZ

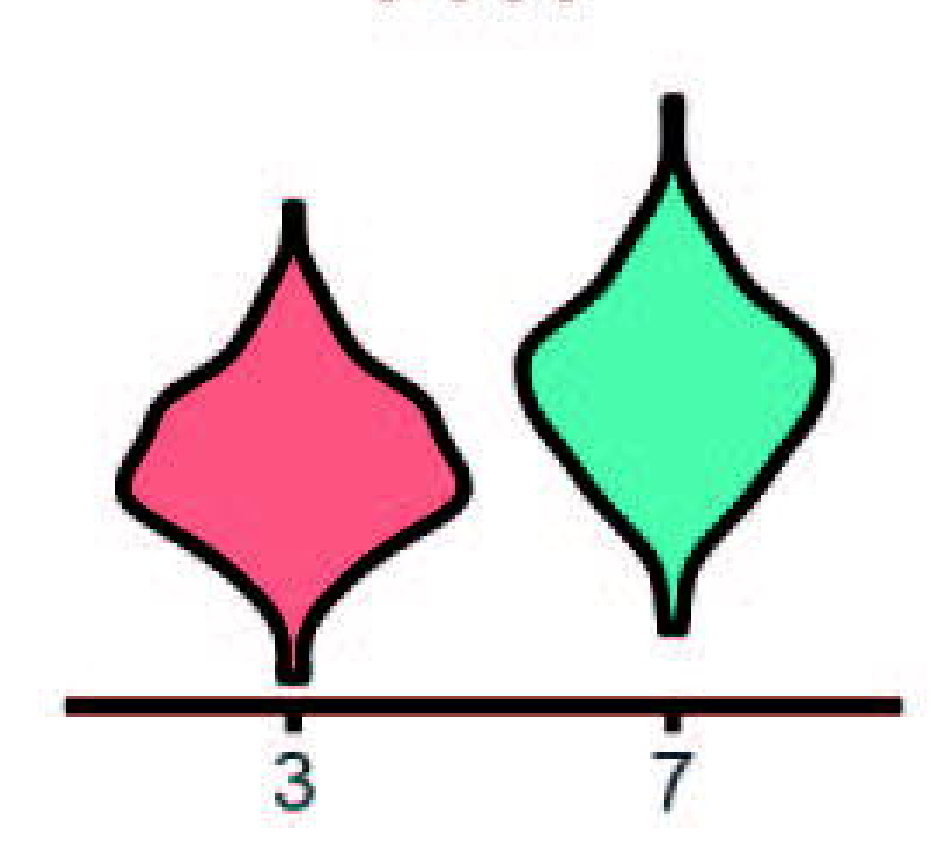

CTSB

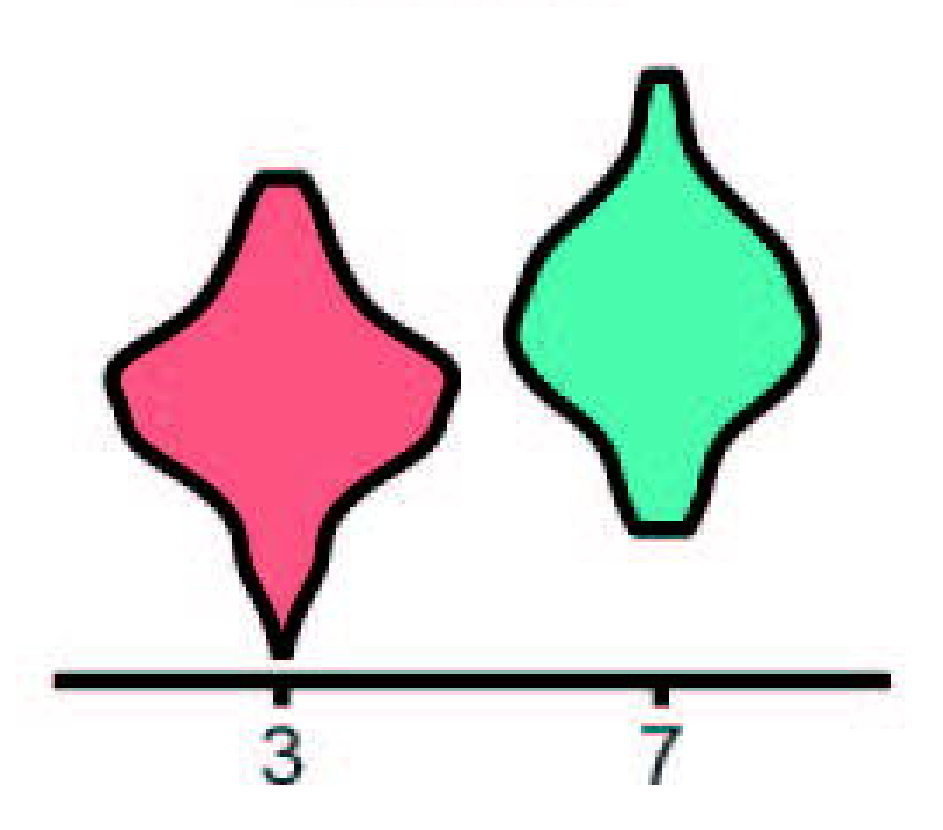

CTSD
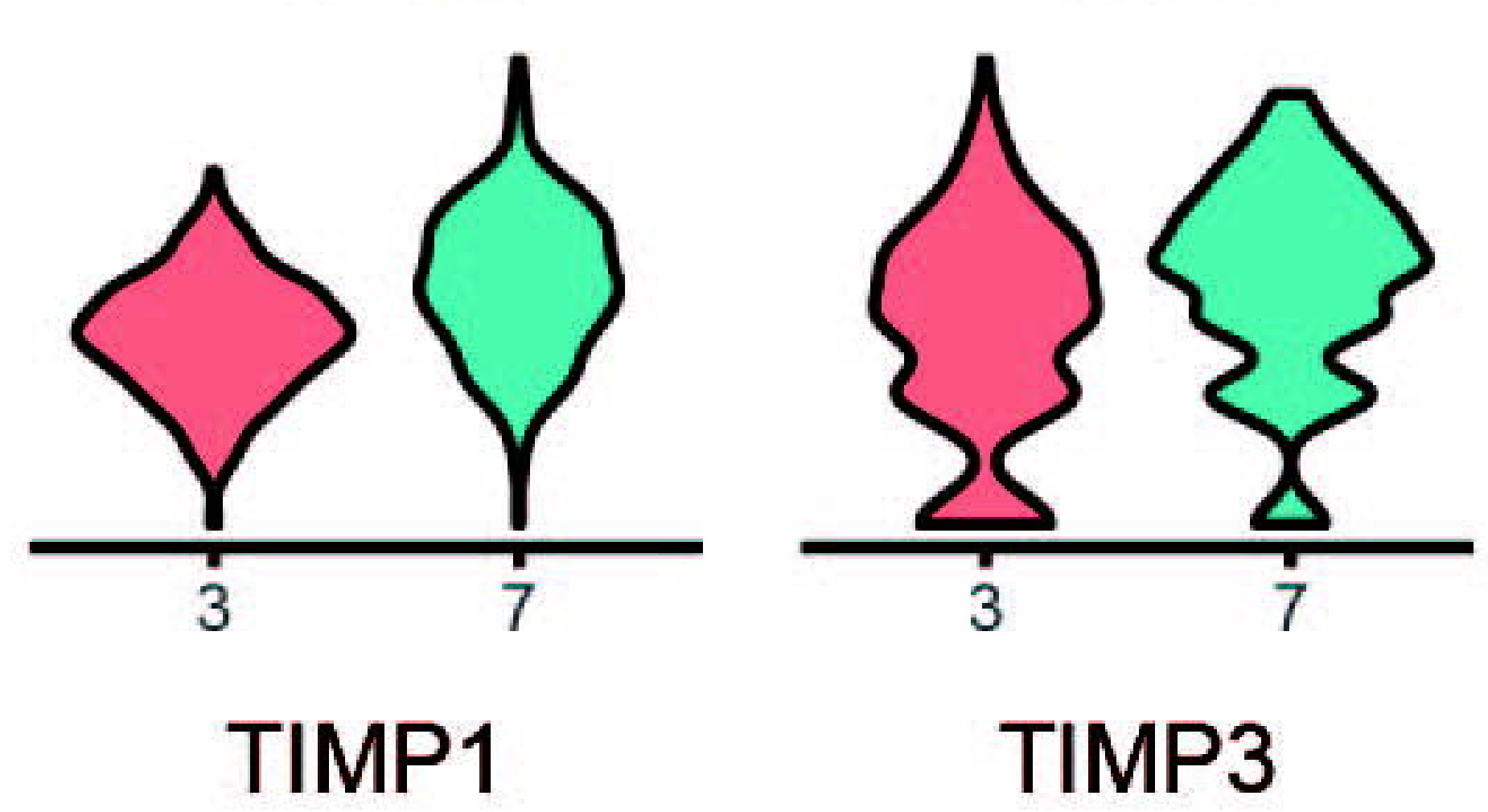

C1QA
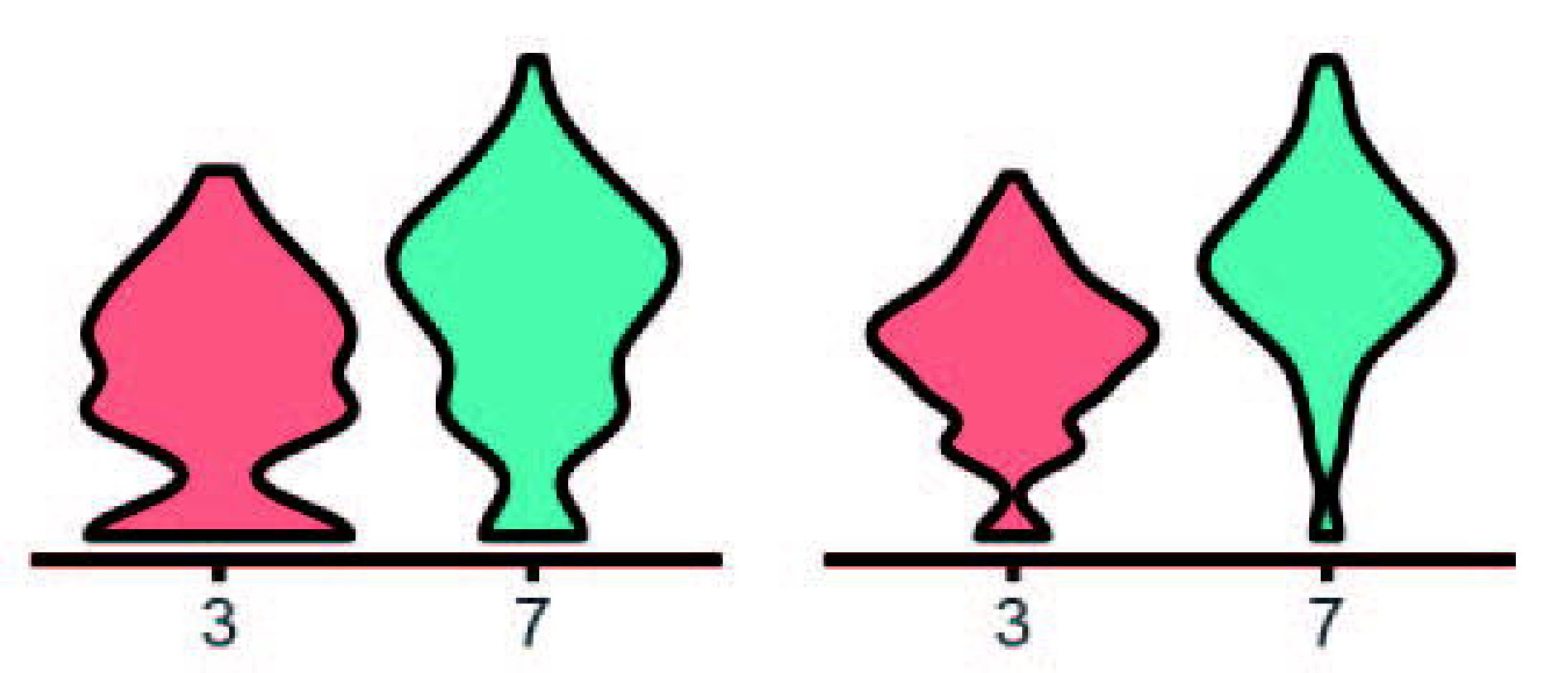

TIMP3

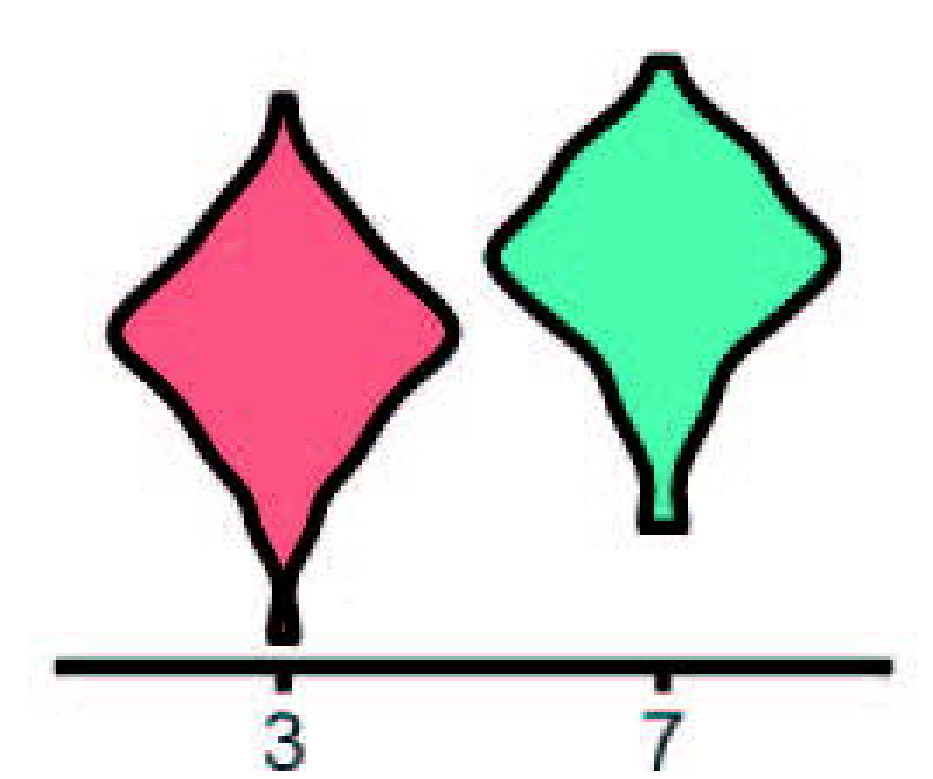

FCGR3B

TNFSF10

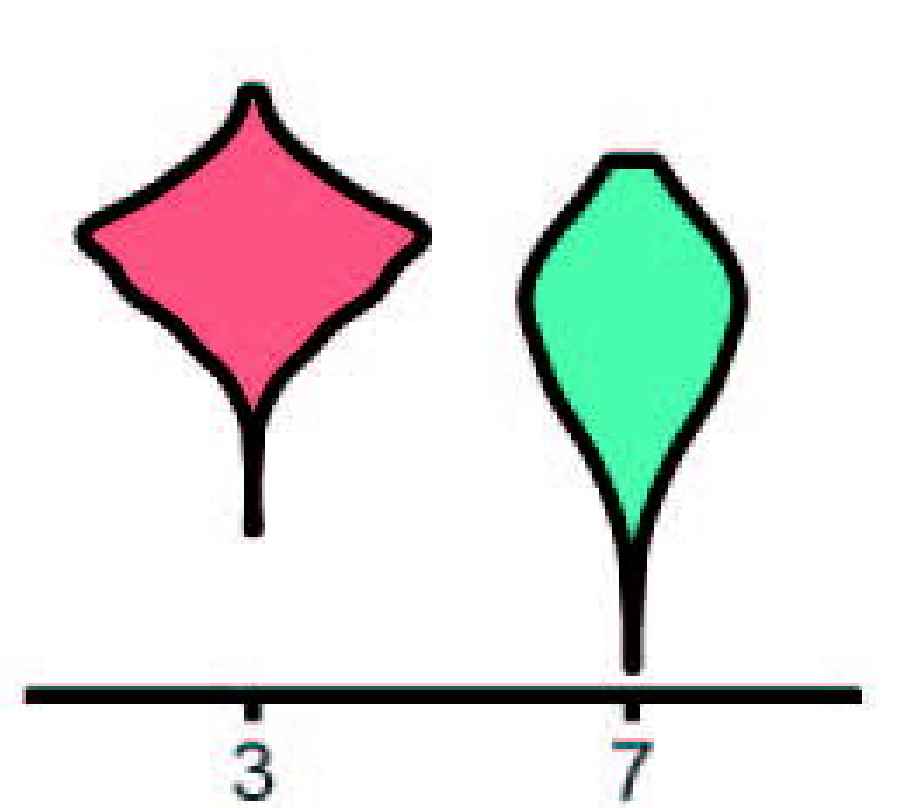

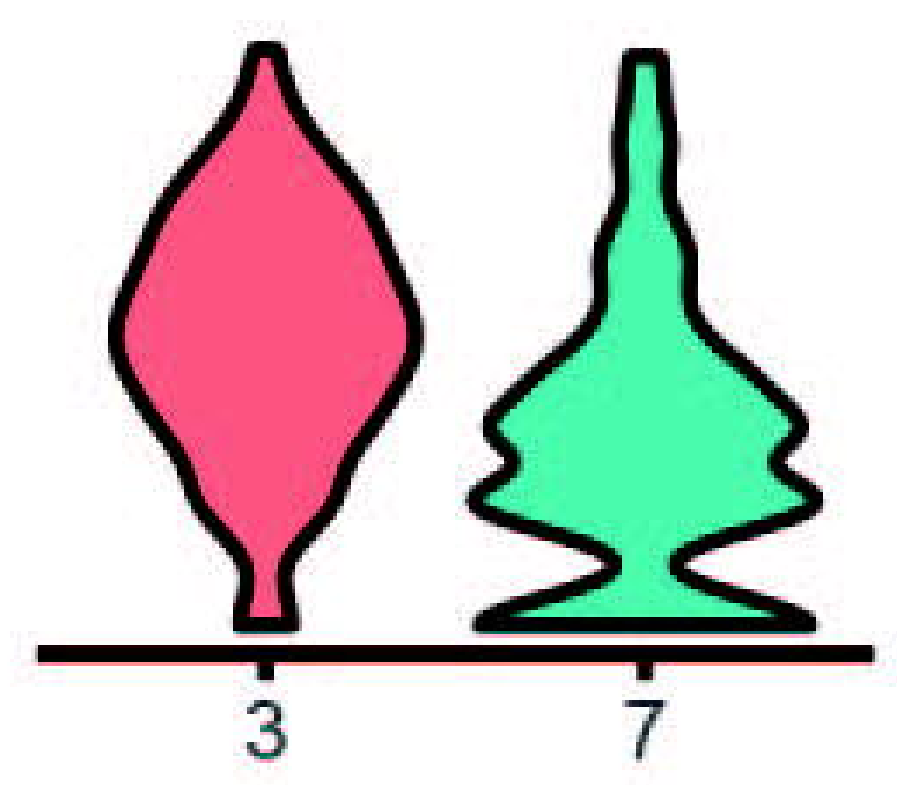
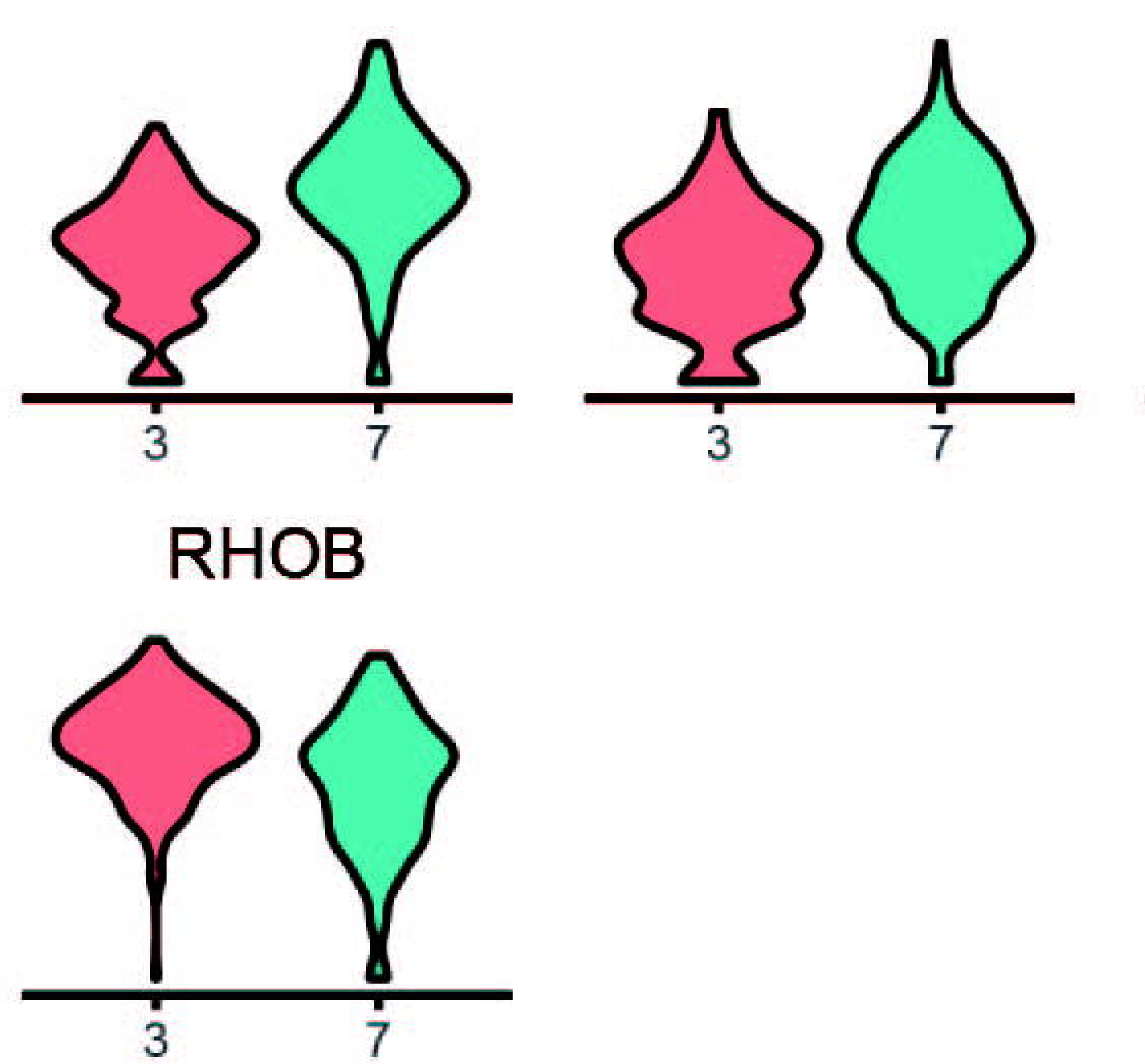